SIMULATION OF PHASE SEPARATION IN

QUIESCENT AND SHEARED LIQUIDS

Amol Kumar Thakre 
Promotion committee:

$\begin{array}{ll}\text { Prof. Dr. J.L. Herek } & \text { University of Twente (chairman and secretary) } \\ \text { Prof. Dr. W.J. Briels } & \text { University of Twente (promotor) } \\ \text { Prof. Dr. G. Gompper } & \text { Forschungszentrum Jülich GmbH } \\ \text { Prof. Dr. M.A.J. Michels } & \text { Eindhoven University of Technology } \\ \text { Prof. Dr. A. van Blaaderen } & \text { University of Utrecht } \\ \text { Prof. Dr. F.G. Mugele } & \text { University of Twente } \\ \text { Prof. Dr. G.J. Vancso } & \text { University of Twente } \\ \text { Dr. Ir. W.K. den Otter } & \text { University of Twente (referee) }\end{array}$

Thakre A. K.

Simulation of phase separation in quiescent and sheared liquids

Thesis, University of Twente, Enschede

This project is supported with a grant of the Softlink program of FOM (Stichting voor Fundamenteel Onderzoek der Materie) which is financed by industry, the national government and the involved institutes and universities.

ISBN: 978-90-365-2719-4

Copyright(C) 2007 by A. K. Thakre the Netherlands

No part of this work may be reproduced by print, photocopy or any other means without the permission in writing from the author.

Front Cover: Phase separated configurations for binary liquid mixture in various Couette cells (A Couette cell is a concentric cylindrical geometry).

Back Cover: Phase separation in polymer/solvent (top) and liquid-crystal/solvent (bottom) mixture, in a micro-Couette cell.

Printed by: Print Partners Ipskamp, www.ppi.nl P.O. Box 333, 7500 AH Enschede, The Netherlands 


\title{
SIMULATION OF PHASE SEPARATION IN \\ QUIESCENT AND SHEARED LIQUIDS
}

\author{
DISSERTATION
}

to obtain

the degree of doctor at the University of Twente,

on the authority of the rector magnificus,

prof. dr. H.W.M. Zijm,

on the account of the decision of the graduation committee

to be publicly defended

on Friday, 12 September 2008 at 16.45

by

\section{Amol Kumar Thakre}

born on 25 may 1978

in Amla, India 
This dissertation has been approved by the promotor:

Prof. dr. W.J. Briels (promotor) 


\section{to my parents ...}

for providing constant inspiration to excel in life 



\section{Contents}

1 Introduction 1

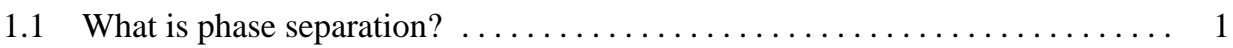

1.2 Background and scope of the research $\ldots \ldots \ldots \ldots \ldots \ldots \ldots \ldots \ldots \ldots .2$

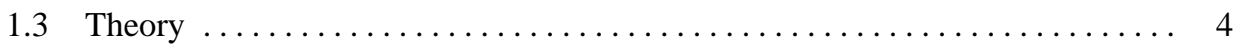

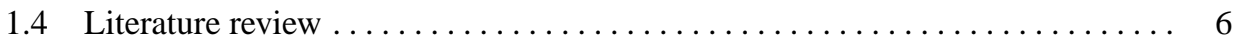

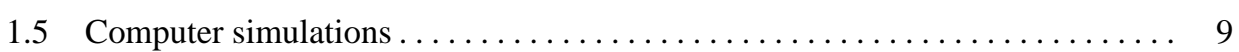

1.6 Thesis outline ...................................... 11

2 Domain formation and growth in spinodal decomposition 13

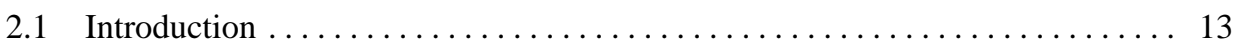

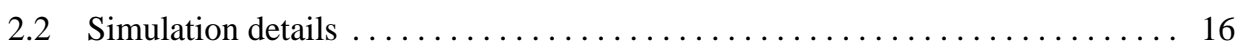

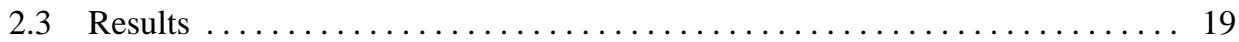

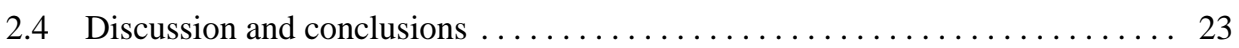

3 Phase separation of binary liquids in cylindrical Couette flow 27

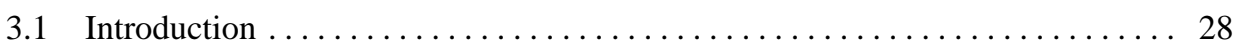

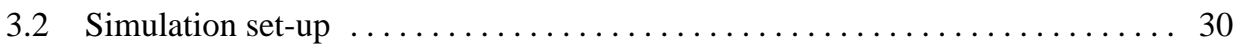

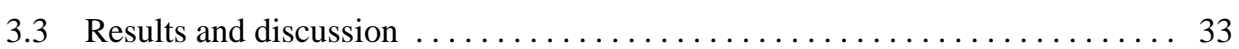

3.3.1 No shear .................................. 33

3.3.2 With shear, starting from phase separated systems $\ldots \ldots \ldots \ldots \ldots . \ldots 35$

3.3.3 With shear, starting from randomly mixed systems ............ 38

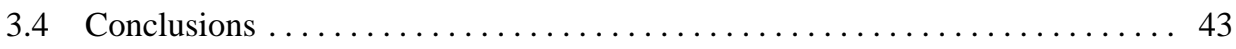

Appendix A: Interfacial free energy in a Taylor-Couette geometry ............ 44

Appendix B: Derivation of Eqs. (3.7) and (3.8) . . . . . . . . . . . . . . . 49

4 Spinodal decomposition in asymmetric binary fluids 51

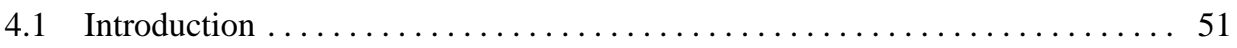

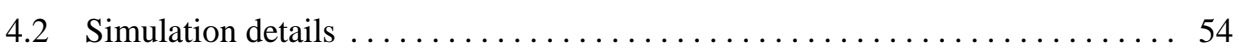


4.3 Results $\ldots \ldots \ldots \ldots \ldots \ldots \ldots \ldots \ldots \ldots \ldots \ldots \ldots \ldots \ldots \ldots \ldots \ldots$

4.3.1 Characterization of the fluids $\ldots \ldots \ldots \ldots \ldots \ldots \ldots \ldots \ldots \ldots \ldots \ldots \ldots \ldots \ldots \ldots$

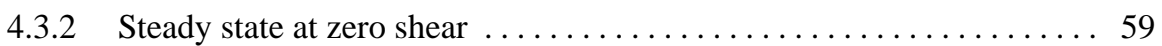

4.3.3 Decomposition dynamics at zero shear $\ldots \ldots \ldots \ldots \ldots \ldots \ldots \ldots$

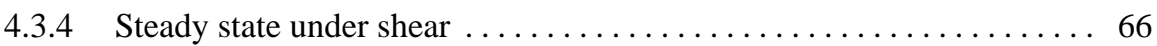

4.3.5 Decomposition dynamics under shear ................ 73

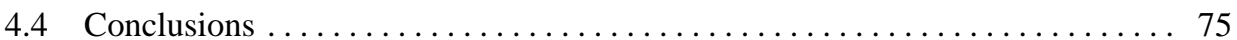

Appendix A:Shear stress $\tau_{r \theta}$ in a Couette geometry $\ldots \ldots \ldots \ldots \ldots \ldots \ldots$

5 Effect of confinement on the interfacial dynamics of binary liquid films 81

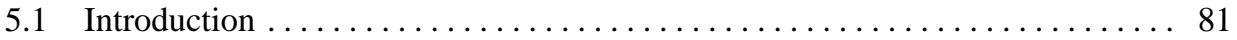

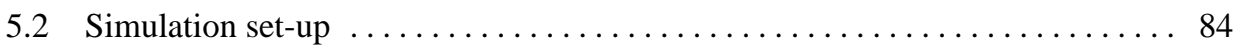

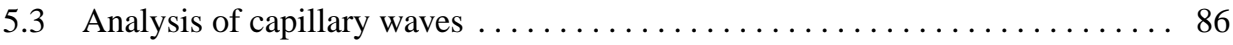

5.4 Results $\ldots \ldots \ldots \ldots \ldots \ldots \ldots \ldots \ldots \ldots \ldots \ldots \ldots \ldots \ldots \ldots \ldots \ldots$

5.4.1 Characterisation of the fluids $\ldots \ldots \ldots \ldots \ldots \ldots \ldots \ldots \ldots \ldots \ldots \ldots \ldots$

5.4.2 Amplitudes of interfacial fluctuations ................. 88

5.4 .3 Dynamics of interfacial fluctuations $\ldots \ldots \ldots \ldots \ldots \ldots \ldots$

5.4 .4 Influence of shear flow $\ldots \ldots \ldots \ldots \ldots \ldots \ldots \ldots \ldots \ldots \ldots \ldots \ldots \ldots$

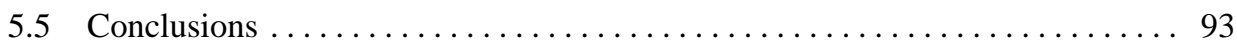

Appendix A: Flow field between solid walls and a fluctuating interface $\ldots \ldots \ldots 95$

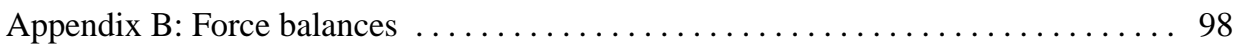

6 Conclusions and Outlook 103

$\begin{array}{ll}\text { Samenvatting } & 107\end{array}$

$\begin{array}{ll}\text { Bibliography } & 110\end{array}$

$\begin{array}{ll}\text { Acknowledgments } & 117\end{array}$

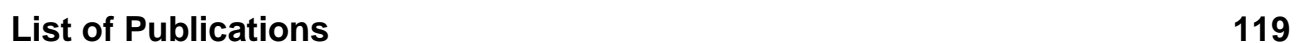

$\begin{array}{lr}\text { About the author } & 120\end{array}$ 


\section{Introduction}

This chapter introduces phase separation and its applications in various fields. Subsequently it discusses the factors which make it an interesting research problem. A brief overview of theory and literature review is presented in the next sections. Finally, simulation approach adopted in this work is presented along with the outline of the thesis at the end.

\subsection{What is phase separation?}

A phase is a homogeneous region of matter having uniform chemical composition and physical properties. In a liquid mixture, unfavourable interactions between the individual liquid components are responsible for the phenomenon of phase separation. A very common example of phase separation can be observed in daily life in curdling of milk after addition of lemon juice. Milk separates in two different components by phase separation; liquid whey and solid curd. In Figure 1.1 two different liquid mixtures are undergoing separation starting from a well mixed condition. The phenomenon of phase separation is observed in various kinds of condensed matter ranging from metals, semiconductors, superconductors to simple and complex fluids such as polymers, surfactants, colloids, emulsions and biological materials. Ordering and pattern formation in any physio-chemical system is a consequence of phase separation, and hence phase separation dynamics has been extensively studied in the past few decades. Despite of a great interest from scientific and technological fronts, understanding of phase separation still eludes scientists and a complete description of the various stages of 
phase separation is still lacking.
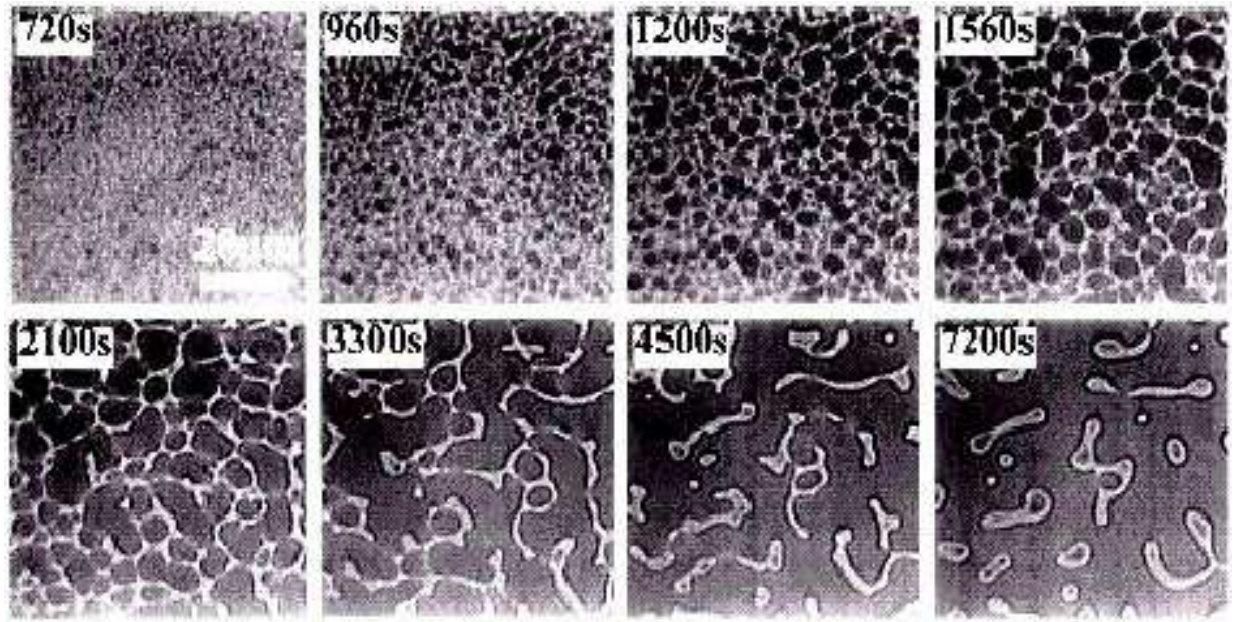

Figure 1.1: Experimental pictures of phase separation in a polymeric mixture (reproduced with permission from $\mathrm{H}$. Tanaka)

\subsection{Background and scope of the research}

When a binary mixture of liquids is quenched below its critical point, the mixture undergoes a phase separation and depending on the ratio of the phases, various patterns evolve. Thermodynamics provides the driving force to reach the final equilibrium state of the system while the components dynamics is means by which the system reaches there. In figure Fig. 1.2 (left) a free energy curve for a symmetric binary mixture is shown, where minimum represents a stable phase. In case of a mixture at high temperature there is only one minimum (bottom), at low temperature there are two (top). The system trapped in between binodals wants to split, which it will do immediately (between spinodals) or after a nucleation event (between spinodals and binodals). The temperature dependence of binodals and spinodals are shown in Figure 1.2 (right).

In case of a 50-50 or symmetric (on basis of volume fraction) mixture a continuous pattern is observed but off-symmetric cases form a minority and a majority phase. This pattern 
formation is due to various stages in dynamics of phase separation. The early stage of phase separation is due to diffusion where molecules move due to thermal motion and form small clusters. In the next stage these clusters collectively make an interface and the dynamics of phase separation is driven by the hydrodynamic flow fields. The third and final stage of phase separation is due to large scale structures which are responsible for non-linear flow fields.

The rate of pattern formation can be characterised quantitatively and the growth exponent of patterns can be associated with the different stages in the phenomenon of phase separation. Experimentally the average pattern size is measured by light scattering, we use a similar technique in our simulations.
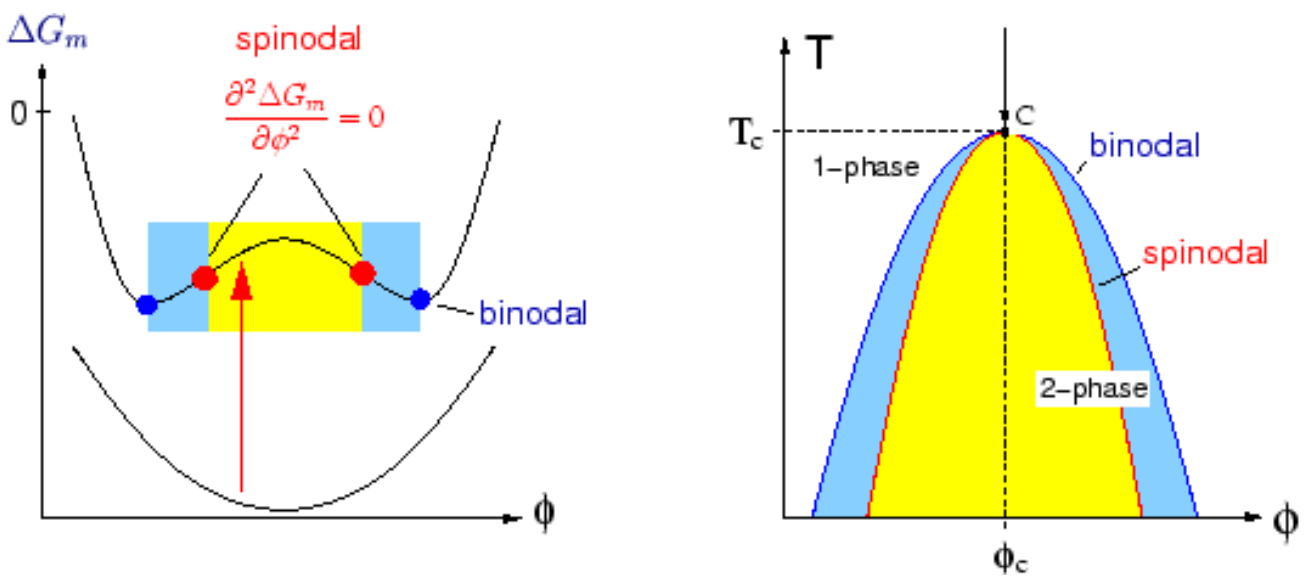

Figure 1.2: Free energy curves for a single phase (bottom left) and two phase system (top left).

Schematic phase diagram, showing binodal and spinodal regimes for a two phase system (right). (Extracted from the web)

Although the phenomenon of phase separation has been observed for decades, a complete theory describing the various stages is still out of reach. The dynamics of phase separation is non-linear and it involves a moving interface, making it a challenging problem to describe analytically and solve. The use of computer simulations has grown significantly over the past years to understand and solve the problem numerically, but the long time and length scales involved in the phenomenon make it inherently difficult to study by a single simulation model. On one hand it involves diffusion, where the time scale associated is of the order of 
$10^{-15} \mathrm{~s}$ and on the other hand later stages are driven by hydrodynamics with time scales of $10^{-6}-10^{-3} \mathrm{~s}$. The same is true for the length scales involved, which vary from nanometers to order of centimeters.

The current research aims to provide an improved fundamental understanding of the physics in the phenomenon of phase separation of simple and complex liquids. The method we use to simulate various stages of phase separation is molecular dynamics, which is based on Newton's second law of motion and described in detail in the section on simulations. Apart from simple liquids, we extended the simulation method to study phase separation in polymers and liquid crystals, which are increasingly interesting to various technological applications because of their self-organisation and ordering behaviours.

\subsection{Theory}

The emerging morphologies of phase separating systems are self-similar in time and are characterised by the emergence of a single time dependent length scale, known as the characteristic length $L(t)$. This length is a measure of the average domain size in the system and is generally believed to follow a simple power-law dependence with time, $L(t) \sim t^{\alpha}$, where $\alpha$ is the power-law growth exponent. The motion of fluid undergoing phase separation is governed by coupled Navier-Stokes and Cahn-Hilliard equations together known as model $\mathrm{H}[8,34,88]$, which are difficult to solve analytically as they are nonlinear and the phenomenon involves a moving boundary with a non-constant topology.

An important parameter in the description of phase separating mixtures is the order parameter field, measuring the local composition, defined as

$$
\phi=\frac{\rho_{A}-\rho_{B}}{\rho_{A}+\rho_{B}},
$$

where $\rho_{x}$ measures the local density of particles of type $x$. Theoretical studies of pattern growth are based on the time evolution of the order parameter field. The Cahn Hilliard equation for the order parameter reads as

$$
\frac{\partial \phi}{\partial t}+\mathbf{v} \cdot \nabla \phi=\lambda \nabla^{2} \mu
$$

where the transport coefficient $\lambda$ is an Onsager coefficient relating the current of $\phi$ to the gradient of the chemical potential $\mu$. The flow field $\mathbf{v}$ in this equations follows from the 
Navier-Stokes equation

$$
\rho\left(\frac{\partial \mathbf{v}}{\partial t}+(\mathbf{v} \cdot \nabla) \mathbf{v}\right)=\eta \nabla^{2} \mathbf{v}-\nabla p-\phi \nabla \mu
$$

The last term is due to the chemical potential gradient in the fluid, which in a liquid-liquid system essentially gives rise to surface tension. This coupled set of equations is difficult to solve, and no analytical solution is available. Based on the concept of the self-similar growth of the domains, however, a simple dimensional analysis gives a reasonable picture of the scaling laws involved in the problem.

If there is only one characteristic length scale $L(t)$ in the system, then all lengths in the system can be scaled with it. To compare the relative importance of various transport mechanisms, the equations can be scaled in terms of non-dimensional terms. The velocity can be scaled as $v^{*}=v /(L / t)$, the gradient $\nabla \sim 1 / L$ and the chemical potential as $\mu^{*}=\mu /(\sigma / L)$, where $\sigma$ is surface tension, the scaling of Cahn Hilliard equation gives

$$
\begin{aligned}
\frac{1}{t}\left(\frac{\partial \phi}{\partial t^{*}}+v^{*} \cdot \nabla \phi\right) & =\frac{\lambda \sigma}{L^{3}} \nabla^{2} \mu^{*} \\
\frac{\partial \phi}{\partial t^{*}}+v^{*} \cdot \nabla \phi & =\frac{1}{P e} \nabla^{2} \mu^{*}
\end{aligned}
$$

Here $P e$ is the Peclet number which gives the ratio of convective to diffusive transport in the system, $P e=L^{3} / \lambda \sigma t$. In a similar way scaling the Navier-Stokes equation gives

$$
\begin{aligned}
\frac{\rho L}{t^{2}}\left(\frac{\partial v^{*}}{\partial t^{*}}+v^{*} \cdot \nabla v^{*}\right) & =\frac{\eta}{L t} \nabla^{2} v^{*}-\frac{\sigma}{L^{2}} \phi \nabla \mu^{*} \\
\frac{\partial v^{*}}{\partial t^{*}}+v^{*} \cdot \nabla v^{*} & =\frac{\nabla^{2} v^{*}}{R e_{\lambda}}-\frac{\phi \nabla \mu^{*}}{R e_{\sigma}}
\end{aligned}
$$

Here $\operatorname{Re}_{\lambda}=\rho L^{2} / \eta t$ is the traditional Reynolds number, which is the ratio of inertial to viscous terms and $R e_{\sigma}=\rho L^{3} / \sigma t^{2}$, is another non-dimensional number, which denotes ratio of inertial to interfacial terms. The interplay of these terms governs the scaling regimes and the growth exponents in the phase separating binary system. In these equations all the scaled terms are of the same order with the non-dimensional prefactors, numbers showing the relative importance of each term. Since the characteristic length scale in the phase separating system grows with time, all these non-dimensional terms are time dependent.

At very early times the diffusion is dominant and the advection term in the equation 1.4 is negligible. The growth law for the characteristic length is then given by the Binder-Stauffer 
mechanism $L(t) \sim(\lambda \sigma t)^{1 / 3}$. This can be obtained from Eq.1.4 for $P e \sim 1$. At later stages when an interface has formed, coupling between the order parameter field and the velocity field becomes important. The material flow in such system is from regions of high curvature to regions of low curvature. The balance between interfacial and viscous forces gives rise to scaling law $L(t) \sim(\sigma t / \eta)$, which can be seen from Eq.1.5 by equating the prefactors in viscous and interfacial terms, $R e_{\lambda} \sim R e_{\sigma}$. In most experiments the Reynolds number is very low and hence the growth mechanism is always in this viscosity dominated stage. Theoretical arguments predict for even later times an inertia dominated scaling law $L(t) \sim\left(\sigma t^{2} / \rho\right)^{1 / 3}$, which can be obtained from $R e_{\sigma} \sim 1$, but this has not yet been observed experimentally. By comparing the three fore-mentioned scaling laws, one predicts transitions between successive power exponent at lenght scales of $L(t) \approx(\lambda \eta)^{1 / 2}$ for viscous hydrodynamics and $L(t) \approx$ $\left(\eta^{2} / \rho \sigma\right)$ for inertial hydrodynamics.

\subsection{Literature review}

Although the universality of the asymptotic scaling laws is well accepted, most of it is derived from dimensional arguments, and an adequate theory describing the complete dynamics of the phenomenon is still lacking. Most of the experimental studies [49, 99, 100] on phase separating liquid-liquid binary symmetric mixtures are performed at a very shallow quench from the critical temperature, hence the associated Reynolds number is very low and the observed scaling is in the viscous region corresponding to $\alpha=1$. Since phase separation in binary mixtures is a fast process, the critical slowing down in the vicinity of the critical point helps to facilitate the study. For the same reason, liquids with high $\left(10^{3}-10^{5}\right)$ Schmidt's number are preferred, as they allow a large separation of time and length scales between different regimes.

Computer simulations of spinodal decomposition using dedicated Navier-Stokes solvers, such as lattice gas automata and lattice Boltzmann (LB) methods, have confirmed the existence of both linear $\alpha=1[2,46,69]$ and sub-linear $\alpha=2 / 3[4,46]$ growth laws. The transition between both regimes was first studied by Kendon et al. $[45,46]$ by performing LB simulations of one fluid mixture over a range of viscosities, thus effectively and efficiently sampling a far wider range of time and length scales than can be accessed by a single sim- 
ulation of a large system. Phase separation of binary fluid mixtures has also been simulated using off-lattice particle-based methods, like molecular dynamics (MD) $[42,53,59]$ and dissipative particle dynamics (DPD) $[15,41]$. Because of the extremely soft interaction potentials in DPD, a linear growth regime is easily reachable in simulations, especially in the computationally less-demanding two-dimensional systems [15]. The early MD simulations of a binary Lennard-Jones fluid by Ma et al. [59] were also reported to have reached the inertial regime. Laradji et al. [53] simulated a larger Lennard-Jones system and argued, based on a different analysis of the data, that the growth rate is in the viscous regime instead. The short-lived diffusive growth regime has thusfar attracted little attention in the simulations of fluid-fluid spinodal decomposition, which is rather surprising.

In case of sheared liquid mixture an additional time scale of deformation is introduced. Theoretically, the case of phase separating liquids under shear is very complex due to the coupling of bulk flow with the hydrodynamic modes of the interface. Shear flow is found to introduce anisotropy in the growth law of the domains. The exponent $\alpha$ obtained by renormalization group theory is greater than 1 in the velocity (parallel) direction and close to $1 / 3$ in the gradient (perpendicular) direction [14]. This suggests that shear enhances the growth in the velocity direction but suppresses it in the gradient direction. Phase separating binary liquids have mostly been studied by lattice Boltzmann (LB) [31, 41, 45, 82, 84, 98] and by molecular dynamics (MD) $[53,59,68,89,91-93,96,101]$ simulations. Most of the experimental studies are performed in Couette geometries while simulations are in planer geometries. A good comparative simulation study should also incorporate the effect of nonlinear shear, which is a motivation to perform simulations in a Taylor-Couette geometry.

The dynamics of phase separation in more complex liquids, i.e. polymers, liquid crystals and lipids, is very different as compared to simple liquids due to elastic effects and entanglements in high concentration polymer solutions. This leads to a complex network-like structure at an early stage of phase separation and can result in a non-universal growth exponent [88]. Dynamical asymmetry of the two mixed fluids, meaning that their viscosities differ significantly or that one component shows viscoelastic behaviour, explains the complex phase separation processes and the 'phase inversion' phenomenon observed in polymersolvent mixtures and colloidal suspensions $[5,86,88]$. In these systems the 'slow' component can not keep up with the growth rate imposed by the 'fast' component, a network enriched in 
the slow component forms (see Fig. 1.1) and subsequently succumbs to internal stresses to arrive again at a regular phase separated final configuration. An externally imposed shear flow both accelerates and hinders the phase separation process in complex liquids, by the continuous transport, elongation and resulting ruptures of the domains [11,67]. In combination with the interfacial driving force, these give rise to anisotropic growth of the domains, with two linear growth processes in the flow-vorticity plane and a possibly supralinear growth, $\alpha>1$ (not observed in case of simple liquids), in the shear gradient direction [11, 14, 54]. Another interesting spinodal decomposition process has been reported for rod-like colloids, where the nematic (dis)ordering enslaves the spatial ordering into dense and less-dense phases $[19,56]$.

In a completely separated binary mixture, the interface is not completely smooth due to thermal undulations. For ordinary molecular liquids, with surface tensions in the $\mathrm{mN} / \mathrm{m}$ range, the capillary roughness is relatively small, in the order of a few or tens of Ångstroms, and can be accessed experimentally by ellipsometry [7,61], X-ray scattering [23,60,75] and neutron $[39,55,76]$ reflectometry. Interestingly, in a recent series of papers $[1,18,73]$, Aarts and coworkers focused on colloid-polymer mixtures in which the interfacial tension between polymer lean and polymer rich phases is lowered to the $\mathrm{nN} / \mathrm{m}$ range. Because of this ultralow surface tension, the characteristic length and time scales of the interfacial fluctuations are such (micrometers and seconds, respectively) that they can be studied in real space by means of confocal microscopy. An often used approximation in capillary wave theory [9, $13,72]$ is that the wavelength of the fluctuation under investigation is much smaller than the thickness of the liquid layer below the interface and, in case of two liquids, also much smaller than the thickness of the liquid layer above the interface. In many experimental situations this approximation is valid and greatly simplifies the theoretical analysis [62]. Although in simulation work much attention has been given to equilibrium properties of the interface, such as molecular organisation and structure and density profiles [65,71,78,83,94], no direct measurements have been reported of the dynamics of capillary interfacial fluctuations. 


\subsection{Computer simulations}

Molecular dynamics (MD) is solving Newton's equation of motion on a computer for a multi particle system. In classical Newtonian mechanics, equation of motion can be written as,

$$
\mathbf{F}_{i}=m_{i} \frac{d^{2} \mathbf{r}_{i}}{d t^{2}}=-\nabla_{i} U
$$

where $m_{i}$ is the mass, $\mathbf{r}_{i}$ is the position and $U$ is the total potential energy. This essentially gives the recipe for calculating the velocities and positions at the next step based on their current values, given that energy functional $U$ is explicitly known. $U\left(\mathbf{r}_{\mathbf{N}}\right)$ has only position dependence and calculations are simplified if the potential is assumed to be of binary type. The most popular scheme is the Verlet method, where the velocity is calculated at half step and used to calculate the new positions,

$$
\begin{aligned}
& \mathbf{v}_{i}\left(t+\frac{\Delta t}{2}\right)=\mathbf{v}_{i}\left(t-\frac{\Delta t}{2}\right)+\frac{\mathbf{F}_{i}(t)}{m_{i}} \Delta t, \\
& \mathbf{r}_{\mathbf{i}}\left(t+\frac{\Delta t}{2}\right)=\mathbf{r}_{i}(t)+\mathbf{v}_{i}\left(t+\frac{\Delta t}{2}\right) \Delta t,
\end{aligned}
$$

where $\Delta t$ is the time step for integration. It is obvious that a higher time step would allow faster sampling of the system, however the limit is placed by the numerical accuracy of the integration. The above steps are repeated until the evolution of the system has reached the desired time scale.

Due to limitations of processor speed and storage capacity of the computer, the simulations are performed on a relatively small number of molecules. To minimize the effects of surfaces, periodic boundary conditions are applied. To implement periodic boundary conditions, the central box is repeated in space. Particles leaving from one side appear from the other side of the central box see Fig. 1.3. This repetition of the central box is only virtual, the calculations are performed only on the central box. This method conserves the number of particles in the central box and allows sampling of a bulk system.

Although potential between the particles is long ranged but tail of the potential is relatively unimportant. A cut-off distance, after which the interactions are significantly lower, is used to keep track of the pair list for the particles. The total number of pairs in the system increases as $O\left(N^{2}\right)$ but this removal of tails allow, the algorithm to be of the order of $O(N)$ and hence significantly faster. 


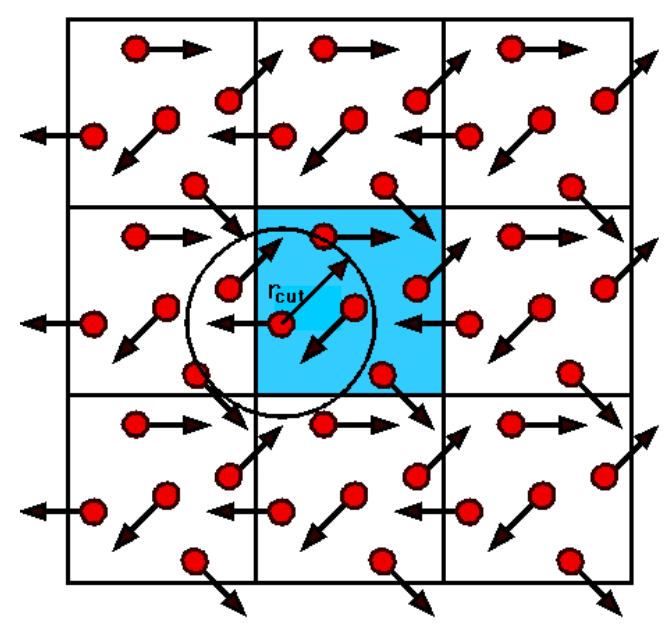

Figure 1.3: Periodic boundary conditions, central box is surrounded by its image in space to simulate a bulk system. (Extracted from the web)

In most of the experiments, the sampling method used is one with constant temperature and not with constant energy, to keep the temperature in a phase separating system a thermostat is needed. Traditional thermostats like Berendsen and Noose-Hoover [24] interfere with the hydrodynamic flow field but Dissipative Particle Dynamics (DPD) thermostat is the one which conserves momentum locally and hence is suited for simulation of hydrodynamic effect. DPD was first introduced by Hoogerbrugg and Koelman [35] and its thermodynamic consistency was verified by Espanol and Warren [29]. We use only the dissipative and random forces given by DPD, but not the soft interaction potential generally used in the DPD. In DPD the friction force $\mathbf{F}_{\text {fric }}$ and random force $\mathbf{F}_{\text {ran }}$ between a pair of particles separated by a distance $r$ within the cutoff distance $r_{c}$ are given by

$$
\begin{aligned}
& \mathbf{F}_{\text {fric }}=-\frac{\kappa 2}{2 k_{B} T}\left(1-\frac{r}{r_{c}}\right)^{2}(\hat{\mathbf{r}} \cdot \Delta \mathbf{v}) \hat{\mathbf{r}} \\
& \mathbf{F}_{\text {ran }}=\frac{\kappa}{\sqrt{\Delta t}}\left(1-\frac{r}{r_{c}}\right) \zeta \hat{\mathbf{r}}
\end{aligned}
$$

where $\kappa$ is the strength of the friction constant, $\hat{\mathbf{r}}$ is the unit vector in the direction of the line joining the two particles, $\Delta \mathbf{v}$ is the velocity difference between the particle pair, and $\zeta$ is a random number with zero mean and unit variance. These dissipative and random forces 
not only act as a thermostat but they also allow transport properties such as viscosity and diffusion to be tuned without altering the equilibrium thermodynamics. Most importantly, all forces are pairwise additive and hence conserve linear and angular momentum.

Computer simulations sit on the interface of theory and experiments; on the one hand they can be used as tools to verify existing theories and results of experiments, on the other hand simulations can be very useful in providing insight at the length and time scales unreachable by experiments or theory. Molecular dynamics simulation is an extremely useful tool for studying various systems at the atomic level, e.g. in the field of biophysics, biochemistry, colloids and soft-matter. It has been used extensively to probe the properties of simple and complex fluids, making it an obvious choice to study the phenomenon of phase separation. The first use of molecular dynamics was Alder and Wainwright [1957,1959], where particles moved with constant velocities between collisions and the binary collisions were perfectly elastic. This idea was then extended to solve the equations of motion for a set of LennardJones particles, with the velocity change derived from the instantaneous force [Rahman 1964] . Since then computer simulations developed very rapidly and now they are extensively used from material science to nanotechnology.

\subsection{Thesis outline}

In this thesis the phenomenon of phase separation in liquid-liquid mixtures (both simple and complex) is studied extensively using molecular dynamics. We investigate growth and growth rates patterns in sheared and non-sheared systems. Each chapter is self contained and can be read independently.

In Chapter 2, we simulate the early diffusive stage of phase separation, which has drawn surprisingly little attention despite the short time and length scales involved in the problem. We extend the investigation to probe the transition from diffusive to viscous growth and the effect of temperature.

In Chapter 3, we study phase separation in a Taylor-Couette geometry, also used in most experimental studies, for sheared and non-sheared systems. In non-sheared cases, starting from a well mixed state and letting the system phase separate, we compare the end configurations obtained by the simulations with the theoretical predictions. A detailed theory for 
calculating free energies with interface being in $r, \theta$ or $z$ direction of a cylindrical coordinate system, is presented in the appendix. Since shear introduces a new deformation time scale in the system, sheared simulations are performed to get a better insight on the effect of interplay between deformation and separation time scale. Results for the effect of shear on initially completely separated system or well mixed systems are presented.

In Chapter 4, we extend the study of previous chapter to more complex liquids. Here, we investigate the effect of dynamic asymmetry on phase separation. We use flexible and liquidcrystalline polymers, modeled by a bead spring model against a simple liquid to introduce the dynamic asymmetry. A polymer chain is modeled by a bead spring model, where spring has a finite extensibility. An additional potential between 1-3 beads is introduced to model the liquid-crystalline polymer. Strikingly different patterns are observed in case of asymmetric binary liquids in shear and non-shear cases, which were not found in simple liquids.

In Chapter 5, we study the effects of finite system sizes and walls on the interfacial dynamics of binary nano-films. The relaxation times of the interfacial modes are measured and compared with the theoretical predictions. We also present a generalized theory including interfacial slip to analyze the amplitude and time correlation of the interfacial modes. Results for the effect of rate of deformation on the relaxation times of various modes are presented. However, a theoretical modeling of the effects still remains an open research question due to non-linearity involved in the problem.

In Chapter 6, the results are summarised in both English and Dutch. 


\title{
2 Domain formation and growth in spinodal decomposition
}

\begin{abstract}
The two initial stages of spinodal decomposition of a symmetric binary LennardJones fluid have been simulated by molecular dynamics simulations (MD), using a hydrodynamics-conserving thermostat. By analysing the growth of the average domain size $R(t)$ with time, a satisfactory agreement is found with the $R(t) \propto t^{1 / 3}$ Lifshitz-Slyozov growth law for the early diffusion-driven stage of domain formation in a quenched homogeneous mixture. In the subsequent stage of viscousdominated growth, the mean domain size appears to follow the linear growth law predicted by Siggia.
\end{abstract}

\subsection{Introduction}

Phase separation and pattern evolution are well known phenomena visible in various immiscible multicomponent mixtures, ranging from simple liquid mixtures to complex fluids like polymers, colloids, surfactants, emulsions and biological materials [30]. Free energy minimalization in combination with hydrodynamic flow, collectively known as model $\mathrm{H}$ [8,34,88], determines the global structure of the emerging pattern and the rate at which it evolves. The bi-continuous morphologies observed in the spinodal decomposition of symmetric binary liquids are commonly believed to be self-similar in time, i.e. the patterns at any two moments in time resemble one another and differ only by a scaling factor. This dynamical scaling hypothesis implies that a single time-dependent characteristic length $R(t)$ can be used to characterise the growth of the pattern. It is generally accepted that this evolution follows a simple power- 
law, $R(t) \sim t^{\alpha}$, where $\alpha$ is the growth exponent. Since the coupled and non-linear differential equations for the composition and flow fields in model $\mathrm{H}$ can not be solved analytically, a comparison of the dominant terms in these equations has led to the identification of three successive growth regimes,

$$
R(t) \propto\left\{\begin{array}{cc}
t^{1 / 3} & (\text { diffusive }) \\
t & \text { (viscous) } \\
t^{2 / 3} & (\text { inertial })
\end{array}\right.
$$

Directly following the spinodal quench of a homogeneous mixture, diffusion is the dominant process driving particles to like particles, culminating in the formation of tiny clusters. The growth law is then given by the Lifshitz-Slyozov mechanism [57], $R(t) \propto(\lambda \gamma t)^{1 / 3}$, where $\lambda$ is a diffusive transport coefficient and $\gamma$ is the interfacial tension of the domain boundaries. After the formation of domains with well-defined interfaces, the minimalization of their interfacial energies becomes the driving force behind segregation. Siggia [79] derived that the balance between interfacial and viscous forces then gives rise to the linear scaling law $R(t) \propto(\gamma t / \eta)$, with $\eta$ the viscosity of the liquid. During this growth the Reynolds number, $R e=(\rho / \eta) R(d R / d t)$ with $\rho$ denoting the specific gravity, steadily increases. This led Furukawa [27] and Kendon [44] to predict an inertia-dominated scaling law, $R(t) \propto\left(\gamma t^{2} / \rho\right)^{1 / 3}$, as the final stage in the growth process. By comparing the aforementioned scaling laws, a transition from the diffusive to the viscous regime is predicted (denoted by an asterisk) to occur at time $t_{d v}^{*} \approx\left(\lambda \eta^{3} / \gamma^{2}\right)^{1 / 2}$ and length $R_{d v}^{*} \approx(\lambda \eta)^{1 / 2}$, while the viscous regime is succeeded by an inertial regime at time $t_{v i}^{*} \approx \eta^{3} /\left(\gamma^{2} \rho\right)$ and length $R_{v i}^{*} \approx\left(\eta^{2} / \rho \gamma\right)$, corresponding to a Reynolds number $R e_{v i}^{*} \approx 1$. These approximate expressions serve as guides in the ongoing experimental and numerical research of spinodal decomposition, to be discussed next, which has largely confirmed the power-law growth of the domains.

In most experimental studies on fluid-fluid phase separation in mixtures of simple liquids $[12,99,100]$, the spinodal decomposition is initiated by a very shallow quench of a homogeneous system to a temperature barely below the critical temperature. The associated Reynolds numbers are very low, hence the observed scaling follows the viscosity-dominated $\alpha=1$ scaling law. Since phase separation usually progresses extremely rapidly, the critical slowing down in the vicinity of the critical point is exploited to facilitate the experiments. Liquid with high Schmidt numbers $\left(S c=\eta /(\rho D)=10^{3}-10^{5}\right.$, with $D$ the diffusion 
coefficient) are preferred for the same reason. With these expedients, the rapid diffusiondominated regime of initial domain formation is just about detectable in mixtures of simple liquids [12, 100]. Suspensions of large unlike particles, such as colloids and polymers [97], also display a diffusive $t^{1 / 3}$ coarsening of the domain size, be it much slower than in fluidfluid mixtures. The inertia-dominated growth regime has not yet been observed experimentally.

Computer simulations of spinodal decomposition using dedicated Navier-Stokes solvers, such as lattice gas automata and lattice Boltzmann (LB) methods, have confirmed the existence of both linear $\alpha=1[2,46,69]$ and sub-linear $\alpha=2 / 3[4,46]$ growth laws. The transition between both regimes was first studied by Kendon et al. $[45,46]$ by performing LB simulations of one fluid mixture over a range of viscosities, thus effectively and efficiently sampling a far wider range of time and length scales than can be accessed by a single simulation of a large system. The turn-over between both growth regimes occurs surprisingly late, centered around $t_{v i} \sim 10^{4} t_{v i}^{*}$, and is protracted over nearly four orders of magnitude in time. In terms of the Reynolds number, this corresponds to the range $1 \lesssim R e_{v i} \lesssim 100$. A further discussion of the inertial regime was presented by Love et al. [58].

Phase separation of binary fluid mixtures has also been simulated using off-lattice particlebased methods, like molecular dynamics (MD) [42,53,59] and dissipative particle dynamics (DPD) $[15,41]$. In the latter method, proposed a decade ago by Hoogerbrugge and Koelman [35], the hard MD potentials between atoms are replaced by extremely soft interactions between 'fluid elements', and an ingenious thermostat is introduced to make all forces consistent with Newton's third law, which also lies at the basis of hydrodynamics. The main advantages of these particle-based simulation methods are that they are not based on presuppositions regarding the dynamics or thermodynamics of the system, and that they include the perpetual thermal noise. This way, the mesoscopic properties of the phase separating system emerge naturally from the simulations rather than being imposed via the simulation algorithm. Because of the extremely soft interaction potentials in DPD, a linear growth regime is easily reachable in simulations, especially in the computationally less-demanding twodimensional systems [15]. Simulations by Jury et al. [41], combining one thermodynamic state point with a range of tuned viscosities, even suggest that the first glimpses of the broad transition to the inertial regime are attainable with DPD. The early MD simulations of a bi- 
nary Lennard-Jones fluid by Ma et al. [59] were also reported to have reached the inertial regime. Laradji et al. [53] simulated a larger Lennard-Jones system and argued, based on a different analysis of the data, that the growth rate is in the viscous regime instead. Both these simulations employed traditional MD thermostats known to interfere with the consistent build-up of the hydrodynamical flow field, while these hydrodynamic interactions are essential for the viscous growth law. Other simulations with stronger perturbations of the flow field have shown that disturbances may impede viscous domain growth $[4,15]$.

The short-lived diffusive growth regime has thusfar attracted little attention in the simulations of fluid-fluid spinodal decomposition, since the focus has been on the two later stages. In the lattice-based methods, which are specifically designed for the long length and time scales, the omission of the initial stage is self-evident. But for the off-lattice particle-based simulations, which by construction are limited to short length and time scales, the absence of studies on the initial $t^{1 / 3}$ regime is rather surprising. In this study, we concentrate on this first stage of spinodal decomposition and the subsequent transition to the viscous regime. We combine the best features of both above discussed particle based simulation techniques, to wit, the realistic hard interactions from MD and the momentum conserving thermostat from DPD. This chapter is organised as follows: in Section 4.2 we briefly describe the employed simulation model and a technique to determine the average size of the emerging domains. Our simulation results are presented in Section 4.3, followed in Section 2.4 by a discussion and comparison with previous studies.

\subsection{Simulation details}

In our molecular dynamics simulations $[3,24]$, the interaction between two like particles at a distance $r$ is modelled by the Lennard-Jones (LJ) potential,

$$
U_{\mathrm{LJ}}(r)=4 \varepsilon\left[\left(\frac{\sigma}{r}\right)^{12}-\left(\frac{\sigma}{r}\right)^{6}\right]
$$

where $\varepsilon$ and $\sigma$ are the strength and radius of the potential, respectively. The potential is smoothly truncated at the cut-off distance $r_{c}=2.5 \sigma$, to eliminate discontinuities in the energy and in the forces. Unlike particles interact by the same LJ potential in the preparatory equilibration runs and by the purely repulsive Weeks-Chandler-Andersen (WCA) potential, 
defined as $U_{\mathrm{WCA}}(r)=U_{\mathrm{LJ}}(r)+\varepsilon$ for $r \leq 2^{1 / 6} \sigma$ and $U_{\mathrm{WCA}}(r)=0$ for $r>2^{1 / 6} \sigma$, during the phase separation simulations. The Verlet leap-frog algorithm is used to numerically integrate Newton's equations of motion with a timestep $\Delta t=0.002 \tau$, where $\tau=\sqrt{m \sigma^{2} / \varepsilon}$ is the natural $\mathrm{LJ}$ unit of time and $m$ is the mass of a particle.

A thermostat is employed to maintain a constant temperature $T$ throughout the simulation, mainly by dissipating the excess energy released by the phase separating system. The traditional MD thermostats based on velocity scaling [3,24] interfere with the evolution of the hydrodynamical flow field, and are therefore less suited for studying processes in which these flow fields might play an important role. We have therefore used a thermostat, introduced by Hoogerbrugge and Koelman [35] as part of the dissipative particle dynamics (DPD) method, which was particularly designed to obey Newton's third law and hence automatically gives rise to permissible flow fields. In a nutshell: any two particles at a distance $r$ within the cut-off radius $r_{c}$ interact by friction and random forces [16, 29, 35]

$$
\mathbf{F}_{\text {thermo }}=-\frac{\kappa^{2}}{2 k_{B} T}\left(1-\frac{r}{r_{c}}\right)^{2}(\hat{\mathbf{r}} \cdot \Delta \mathbf{v}) \hat{\mathbf{r}}+\frac{\kappa}{\sqrt{\Delta t}}\left(1-\frac{r}{r_{c}}\right) \zeta \hat{\mathbf{r}}
$$

where $\kappa$ sets the activity of the thermostat, $k_{B}$ is Boltzmann's constant, $\hat{\mathbf{r}}$ is the unit vector between the two particles, and $\Delta \mathbf{v}$ is their velocity difference. The random numbers $\zeta$ have zero average, unit standard deviation, are independent for every particle pair and are sampled every time step from a distribution without memory. A fluctuation-dissipation theorem relating the variances of the friction and random forces to the desired equilibrium temperature $T$ has been included in the above equation. Note that the thermostating forces do not affect the thermodynamic properties of the Lennard-Jones fluid, but they will slow down the dynamics of the fluid. We take advantage of this corollary by selecting a fairly high friction parameter, $\kappa=3 \varepsilon \tau^{1 / 2} \sigma^{-1}$, to increase the length $t_{d v}$ of the diffusive growth regime. This particular choice decreases the diffusion coefficient of the $\mathrm{LJ}$ particles by a factor of about three relative to the non-thermostatted fluid. A further convenient property of the DPD thermostat is that it couples to the local temperature, as opposed to conventional MD thermostats which act on the overall mean temperature. Note that we have not used the extremely soft potentials cointroduced with the DPD thermostat [35]: these weak interactions lead to fluids with very low Schmidt numbers [29] and consequently reduce the span $t_{d v}^{*}$ of the diffusive growth regime.

All simulations were performed using three-dimensional symmetric binary mixtures, con- 
taining a total of $N=500,000$ particles in a cubic box with periodic boundary conditions. The number density was fixed at $\rho=0.7 \sigma^{-3}$, yielding box sizes $L=89 \sigma$. Every simulation started with the creation of a new homogeneous system, by randomly inserting particles in the simulation box and rejecting all insertions resulting in a large overlap with previously accepted particles. Next, these boxes were thoroughly equilibrated in MD simulations at the desired temperatures of $T=1 \varepsilon / k_{B}, 2 \varepsilon / k_{B}$ and $3 \varepsilon / k_{B}$, using the same Lennard-Jones potential for all interactions to create homogeneous systems. Finally, phase separation was initiated by replacing the LJ interaction between unlike particles by the WCA potential, which effectively instantaneously quenches the simulation boxes to states deeply below the spinodal. All particle coordinates $\mathbf{r}_{j}(t)$ were stored at intervals of $0.2 \tau$ for later visualization and analysis of the phase separation dynamics.

Since the time-dependent average domain size $R(t)$ is the most interesting and natural measure for the progression of the phase separation, we have determined this coarsening function from the structure factors of the stored configurations. The latter are calculated as

$$
S(\mathbf{k}, t)=\langle\hat{\phi}(\mathbf{k}) \hat{\phi}(-\mathbf{k}),\rangle
$$

where the Fourier transform of the order field reads as

$$
\hat{\phi}(\mathbf{k})=\sum_{j=1}^{N} b_{j} e^{i \mathbf{k} \cdot \mathbf{r}_{j}(t)},
$$

with $b_{j}= \pm 1$ depending on the type of particle $j$ and $\mathbf{k}$ a wavevector commensurate with the box dimensions. Along any direction in reciprocal space, the structure factors of a symmetric binary liquid start at $S=0$ for $k=0$, then rise to a maximum $S_{m}$ for wavenumber $k_{m}$ before gradually returning to zero at large wave numbers. Since the structure factors $S(\mathbf{k}, t)$ calculated from a single configuration at time $t$ are rather noisy, we exploited this rotational symmetry to calculate spherically averaged structure factors $S_{\mathrm{sph}}(k, t)$, using a bin-width of $\Delta k=0.017 \sigma^{-1}$. The structure factors of four independent runs were averaged before making a least-squares fit with the scaling function

$$
S_{F}(k, t)=S_{m}(t) \frac{3\left[k / k_{m}(t)\right]^{2}}{2+\left[k / k_{m}(t)\right]^{6}},
$$

proposed by Furukawa [25] on the basis of the limiting behaviours of $S(k)$ at small and large $k$. One readily shows that this function reaches a maximum of $S_{m}(t)$ for the wave 
number $k=k_{m}(t)$. Note that Furukawa's function is consistent with the dynamical scaling hypothesis, which is expected to hold for the evolving phase separated domains. An offset in the wavenumber, introduced as a third fit parameter to improve the quality of fit [95], spoils this scaling invariance and is therefore not recommendable. The characteristic lengths of the domains in our simulations are finally extracted from the peak positions of the fitted functions by $R(t)=2 \pi / k_{m}(t)$. We believe that this route to the average domain size provides a worthwhile alternative to the more common approaches based on the first or second moment of $S(k)$, especially when the structure factors are compounded with noise.

\subsection{Results}

Visual inspection of the stored trajectory files, using the VMD package [37], vividly illustrates the sequence of events in a phase separating fluid mixture. Immediately following the quench, tiny domains of like particles appear throughout the previously homogeneously mixed system. The domains gradually increase in size until their diameters reach a significant fraction of the box dimensions, at which point the simulations are terminated. A quantitative measure of the domain sizes is obtained by calculating the spherically averaged structure factors $S_{\mathrm{sph}}(k, t)$ of the stored configurations, using the procedure outlined in the previous section. Typical results for four distinct times during the phase separation are plotted in Fig. 2.1. In agreement with the visual inspection of the simulation movies, the position $k_{m}$ of the peak gradually shifts towards lower wave numbers with increasing time. The four data sets are fitted reasonably well, see the thick lines in the plot, by the master curve proposed by Furukawa, see Eq. (2.6). A closer inspection reveals that the master curve systematically overestimates the structure factors in the tails at both sides of the peak. We want to emphasize that the quality of the fit function is of minor importance in the current analysis, provided both the emerging pattern and the applied fit function are in agreement with the dynamical scaling hypothesis. An average domain size $R$ is now readily deduced from the peak position $k_{m}$, which is one of the two fit parameters in the Furukawa function. Since it proved difficult to make reliable fits of the structure factors at early times into the decomposition simulation, $t<5 \tau$, where the demixing has not yet yielded well-defined domains and the signal-to-noise ration in the $S(k, t)$ is still unfavourable, we have omitted these earliest times in the following 


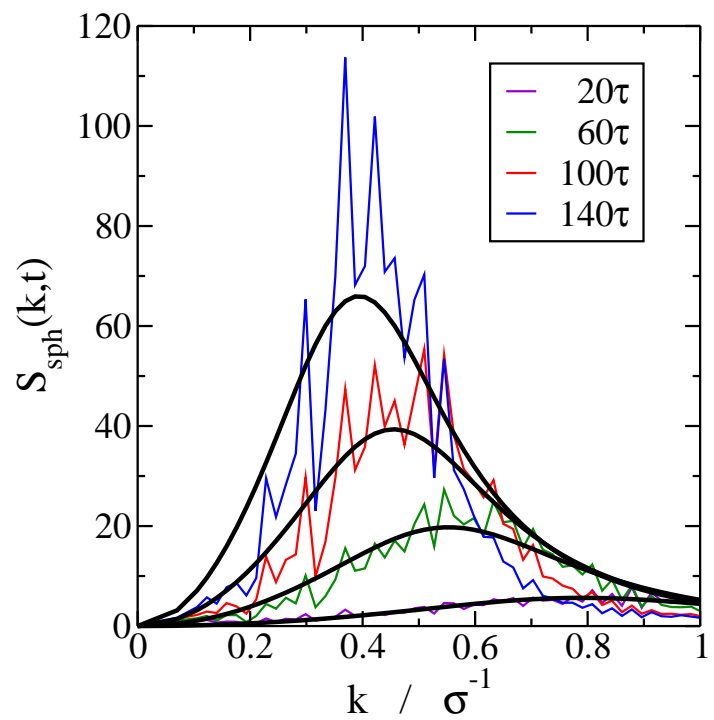

Figure 2.1: Spherically averaged structure factors $S_{\mathrm{sph}}(k, t)$ for four times, $t=20 \tau, 60 \tau, 100 \tau$ and $140 \tau$, after quenching a homogeneous system. The data shown are averages over four independent simulations to improve the signal-to-noise ratio. Thick smooth lines represent fits with the Furukawa function, see Eq. (2.6). As time advances, the position $k_{m}$ of the peak shifts to lower wave numbers, and the height of the peak increases, indicating that the domains are growing.

analysis.

The average domain sizes are plotted as functions of time in Fig. 2.2 for the two lowest temperatures, $T=1 \varepsilon / k_{B}$ and $T=2 \varepsilon / k_{B}$. Both curves show a sub-linear regime at small times followed by a near-linear regime at later times, which we preliminary identify with the diffusive and viscous scaling regimes, respectively. Since the power laws of Eq. (2.1) were deduced from mesoscopic equations of motion, they are expected to hold true for mesoscopic time and length scales only. The current simulations, however, are at a level where the underlying microscopic details might be expected to be still relevant to the domain coarsening. It therefore appears appropriate to fit the observed growth functions with a more general power 


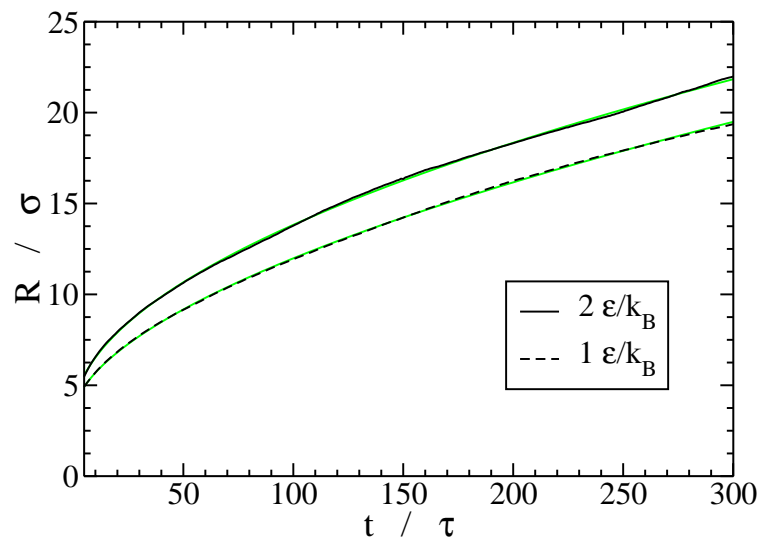

Figure 2.2: Characteristic length scale $R$ of the phase separated domains, as deduced from the structure factors, plotted as a function of time for the two lower temperatures. The green lines are fits with a generalised power law, see Eq. (2.7), whose fit parameters are listed in Table 2.1.

law [53],

$$
R(t)=R_{0}+a(t / \tau)^{\alpha}
$$

where $R_{0}$ represents a microscopic offset in the domain size. The Lennard-Jones unit of time $\tau$ is introduced here for convenience, thus making the dimensions of $a$ independent of the value of $\alpha$. The resulting fit parameters are collected in Table 2.1. Of particular interest are the two similar growth exponents of $\alpha \approx 0.55$, which suggests that we are sampling a time interval close to or surrounding the transition time $t_{d v}$ from the diffusive $\alpha=1 / 3$ to the viscous $\alpha=1$ growth regime. It is tempting, therefore, to extract from the simulated range an initial and a late period; the bounds on these periods are admittedly rather arbitrary in the absence of a clear transition. By fitting the growth curve at $T=2 \varepsilon / k_{B}$ over the initial period $5 \tau \leq t \leq t_{i}$, with $t_{i}=50 \tau, 25 \tau$ and $15 \tau$, we find the substantially lower exponents of $\alpha=0.45,0.39$ and 0.40 , respectively. These values hint at a transient regime with diffusionlimited growth, although the covered time interval is far too short to admit a more definitive conclusion. The other two fit parameters are also fairly consistent in these three regions, with $R_{0} \approx 2.0$ and $a \approx 1.9$. At the lower temperature of $T=1 \varepsilon / k_{B}$, the exponent remains 


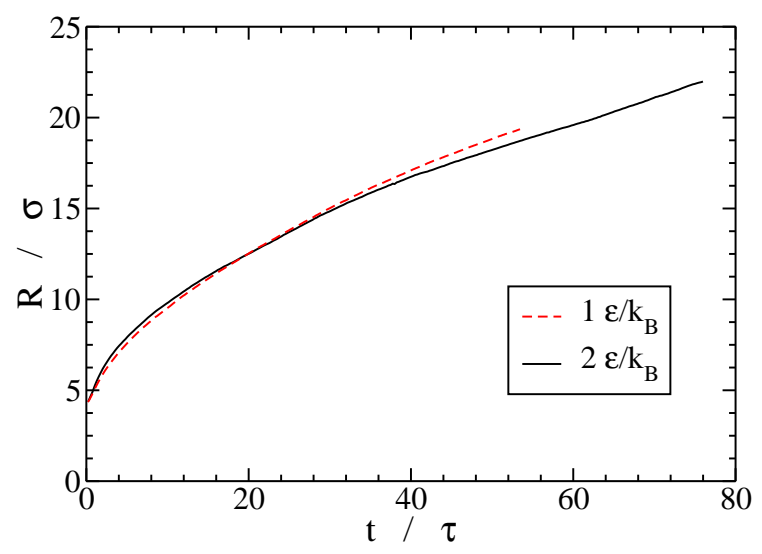

Figure 2.3: Characteristic length scale $R$ of the phase separated domains plotted against scaled time, for the two lower temperatures. The growth curves are expected to coalesce in the viscous regime, after rescaling time with the temperature-dependent factor $\gamma / \eta$.

almost unaltered at $\alpha \approx 0.55$ for all tested upper bounds on the initial range, implying that the diffusive region is extremely short-lived in this case. In the final region of the growth curve, $t_{f} \leq t \leq 300 \tau$ with $t_{f}$ between $150 \tau$ and $250 \tau$, it proves difficult to fit the data with a general power law. The growth exponents are found to vary strongly with $t_{f}$, yielding values as disparate as 0.24 and 1.57 , while the other two fit parameters are equally inconsistent, making the direct evaluation of the growth exponent unreliable in this regime. Laradij et $a l$. [53] identified the viscous growth process in their simulations by noting that rescaling of the curves at different temperatures according to the predicted growth law, $R \propto \gamma t / \eta$, should make the curves coalesce. We calculated the interfacial tension from the difference in the pressures parallel and perpendicular to the fluid-fluid interface in a phase separated box with two flat interfaces $[24,29]$. The viscosity was extracted from the self-diffusion coefficient $D$ in the homogeneous liquid by using the Stokes-Einstein expression, $D=k_{B} T / c \pi \eta R$, with $c=5$ and $R=\sigma / 2$ [10]. Both calculations were performed at $T=1 \varepsilon / k_{B}$ and $2 \varepsilon / k_{B}$. After rescaling both growth curves by their respective factors, we observe the good agreement depicted in Fig. 2.3. Plots of $R(t)$ against $t^{1 / 3}$, the predicted power law in the diffusive regime, yield a less satisfactory agreement between the two curves, even if the value of the 


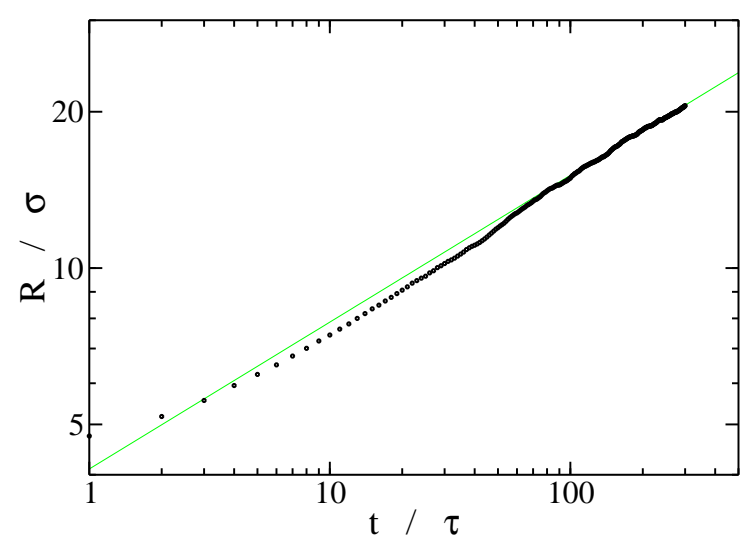

Figure 2.4: Average domain size during spinodal decomposition at the elevated temperature of $T=3 \varepsilon / k_{B}$. The slope $\alpha=0.29$ of the fitted line is in good agreement with the $\alpha=1 / 3$ expected in a diffusive growth process.

undetermined diffusive transport coefficient $\lambda$ is chosen such as to minimize the differences between the curves (not shown). Rescaling both growth curves according to the inertial law produces a clear deviation between the graphs (not shown). Taken together, this strongly indicates that the observed growth of the average domain size is best characterized as being in the viscous regime. A completely different picture emerges at the higher temperature of $T=3 \varepsilon / k_{B}$. Fitting the data with the general power law, see Table 2.1, we find that the average domain size grows with an exponent $\alpha=0.27$ over the entire simulated time interval. On a double logarithmic plot, presented in Fig. 2.4, the curve converges to the straight line $R=4.1 \sigma(t / \tau)^{0.28}$, fitted over the range $100 \tau \leq t \leq 300 \tau$. These exponents are in good agreement with the $\alpha=1 / 3$ predicted for the diffusive regime.

\subsection{Discussion and conclusions}

The initial stages of phase separation in a quenched homogeneous mixture of immiscible binary fluids have been studied by molecular dynamics simulations. At the elevated temperature of $T=3 \varepsilon / k_{B}$, the power-law growth of the average domain size is in good agreement with the initial diffusive growth mechanism $R \propto t^{1 / 3}$ predicted by the Lifshitz-Slyozov the- 
Table 2.1: Parameters obtained by fitting the simulated growth functions at three temperatures with the power law of Eq. (2.7). In the last line, the growth for $t \geq 100 \tau$ was fitted with a simple power law, i.e. without the offset $R_{0}$.

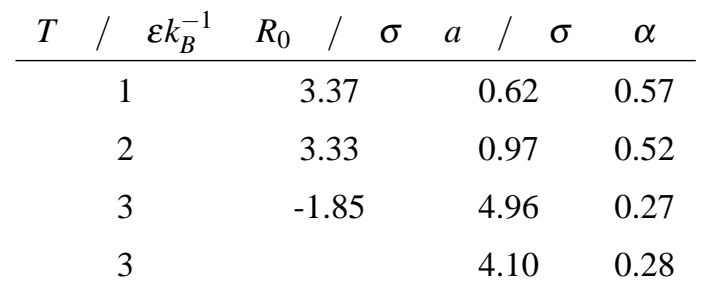

ory. To the best of our knowledge, this is the first clear observation of the diffusive regime in three-dimensional molecular dynamics simulations of spinodal decomposition. For the intermediate temperature of $T=2 \varepsilon / k_{B}$ the diffusive regime is short-lived, and quickly gives way to a viscous regime. The latter is the only regime discernible in the simulations at the low temperature of $T=1 \varepsilon / k_{B}$. Although an accurate growth exponent could not be established, the characterization of this regime is supported by the observed scaling behaviour with temperature.

The small mean domain sizes in the initial parts of the simulations, for $R(t) \lesssim L / 10 \sim$ $10 \sigma$, imply that the simulation boxes contain a large number of domains, be they correlated. In combination with the averaging over four unrelated simulations at every temperature, this suggests that the temporal evolution of the mean domain size has been determined accurately for short times. Since the average repeat distance of the phase separated domains remains relatively small compared to the box dimensions till the termination of the runs, $R(t) \lesssim L / 4$ for all $t$, possible box-size related artefacts are expected to be still of minor importance. The accuracy of $R(t)$ will however decrease at the later times, which might have contributes to the difficulties in determining the growth exponent in the viscous regime.

The introduction of a domain size offset $R_{0}$ in the general power law, as proposed by Laradji et al. [53], is important for the quality of the fit, especially since the actually attained mean domain sizes are not orders of magnitude larger than $R_{0}$. We find that this offset for the two lowest temperatures is of the same magnitude as the sizes of the particles, which agrees 
with the intuition of a viscous growth process starting from small clusters. An additional temporal offset $t_{0}$ was introduced in some fits, replacing $t$ by $t-t_{0}$ on the right hand side of Eq. (2.7). We found that this did not substantially improve the quality of the fit, with $t_{0}$ often being close to zero. A number of fits even became numerically poorly defined, indicative of the redundancy introduced by the extra degree of freedom.

The transition time $t_{d v}$ between diffusive and viscous growth is seen to increase strongly with increasing temperature. At $T=1 \varepsilon / k_{B}$ there is no clear transition, for $T=2 \varepsilon / k_{B}$ we estimate $t_{d v} \sim 50 \tau$, while at $T=3 \varepsilon / k_{B}$ the transition is not even reached within the spanned time range, i.e. $t_{d v}>300 \tau$. We suggest that critical slowing down plays an important role in the pronounced rise of $t_{d v}$ with $T$. Simulations at the higher temperature $T=3.5 \varepsilon / k_{B}$ show a limited transient degree of clustering but do not yield a continuous growth of these clusters, implying that this temperature already exceeds the critical temperature. It can not by ruled out, however, that the effect reflects a steep temperature dependence of $\lambda$. Further calculations, exceeding the scope of this chapter, are required to investigate these possible explanations. A simple calculation yields that $t_{v i}^{*} \approx 100 \tau$ at $T=2 \varepsilon / k_{B}$, which in combination with the previously established $t_{v i} \sim 10^{4} t_{v i}^{*}$ explains why the inertial regime is not observed in the current simulations.

The transition time $t_{d v}$ is also interesting for the study of spinodal decompositions of liquid mixtures exposed to a shear deformation [67,68]. At relatively low shear rates, $\dot{\gamma}<$ $t_{d v}^{-1}$, the effect of the flow is sufficiently small for well-defined domains to form. The flow deformation will distort the domains, possibly tearing them apart, but may also be effective in bringing domains together. For relatively high shear rates, $\dot{\gamma}<t_{d v}^{-1}$, the deformation flow is expected to compete with the diffusive stage of phase separation, thus seriously hindering the formation of clear domains. In the next chapter we will discuss this competition between phase separation and flow deformation, for the current Lennard-Jones liquid, in a Couette geometry. 



\section{Phase separation of binary liquids in cylindrical Couette flow}

We use molecular dynamics simulations to study phase separation of a 50:50 (by volume) fluid mixture in a confined and curved (Taylor-Couette) geometry, consisting of two concentric cylinders. The inner cylinder may be rotated to achieve a shear flow. In non-sheared systems we observe that, for all cases under consideration, the final equilibrium state has a stacked structure. Depending on the lowest free energy in the geometry the stack may be either flat, with its normal in the z-direction, or curved, with its normal in the $r$ or $\theta$-direction. In sheared systems we make several observations. First, when starting from a pre-arranged stacked structure, we find that sheared gradient and vorticity stacks retain their character for the durations of the simulation, even when another configuration is preferred (as found when starting from a randomly mixed configuration). This slow transition to another configuration is attributed to a large free energy barrier between the two states. In case of stacks with a normal in the gradient direction, we find interesting interfacial waves moving with a prescribed angular velocity in the flow direction. Because such a wave is not observed in simulations with a flat geometry at similar shear rates, the curvature of the wall is an essential ingredient of this phenomenon. Secondly, when starting from a randomly mixed configuration, stacks are also observed, with an orientation that depends on the applied shear rate. Such transitions to other orientations are similar to observations in microphase separated diblock copolymer melts. At higher shear rates complex patterns emerge, accompanied by deviations from a homogeneous flow 
profile. The transition from steady stacks to complex patterns takes place around a shear rate $1 / \tau_{d v}$, where $\tau_{d v}$ is the cross-over time from diffusive to viscous driven growth of phase-separated domains, as measured in equilibrium simulations.

\subsection{Introduction}

The kinetics of phase separation in binary liquids has been of a great interest over the years. It is usually described by the time dependence of some characteristic length $R$ in the system, representing the size of the phase separated domains. It has proven difficult to construct a theory describing the time evolution of these length scales, mainly because the dynamics of phase separation is non-linear and involves moving interfaces. Scaling analysis of the Navier-Stokes and Cahn-Hilliard equations present a reasonable picture for different exponents involved in the process in the viscous regime $[8,26,88]$. Based on self similar growth of patterns these predict that the characteristic length scale grows as a power law in time, i.e., $R(t) \propto t^{\alpha}$. The exponent reported for a non-sheared system is $\alpha=2 / 3$ and $\alpha=1$ for two-dimensional and three dimensional binary fluid mixtures respectively. Shear flow causes an additional pattern formation in phase separating mixtures which is very interesting and not completely understood. Pattern formation plays a central role in the formation of various structures in complex fluids such as polymers, colloids, liquid crystals, and self-assembling membranes and micelles. The (shear) rheology of phase separating complex liquids is thus very important from both a physical and an engineering point of view. Theoretically, the case of phase separating liquids under shear is very complex due to the coupling of bulk flow with the hydrodynamic modes of the interface. Shear flow is found to introduce anisotropy in the growth law of the domains. The exponent $\alpha$ obtained by renormalization group theory is greater than 1 in the velocity (parallel) direction and close to $1 / 3$ in gradient (perpendicular) direction [14]. This suggests that shear enhances the growth in the velocity direction but suppresses it in the gradient direction. This seems to be confirmed by experiments [21,32,33,52]. Different time scales are involved in the break up and coalescence of a domain. Depending on the ratio between these time scales and the deformation time scale of the flow, different metastable states are observed. For this no theory is available. Simulations may help in 
gaining insight in the dynamics of phase separating systems. Phase separating binary liquids have mostly been studied by lattice Boltzmann (LB) [31,41, 45, 82, 84, 98] and by molecular dynamics (MD) [53, 59, 68, 89, 91-93, 96, 101] simulations. Many of the early simulations were limited to two-dimensional non-sheared systems, but recent advances in computational power have moved the scope to three-dimensional systems as well. The observed domain growth scaling laws are generally in agreement with the theoretical predictions. During the last decade also simulations of phase separating systems undergoing shear have been carried out, both by $\operatorname{LB}[31,82,84,98]$ and $\operatorname{MD}[68,101]$. In these studies it was observed that the interplay between surface tension and deformation due to shear gives rise to various growth patterns like stretched domains and string phases in the direction of shear. Very recently it has been shown that a non-equilibrium (dynamical) steady state is reached, in which the domains attain a finite length instead of growing indefinitely [31, 82,84]. The finite length is a result of interference of the shear flow with the transition from an interfacial/viscous to an interfacial/inertial regime, and is found to decrease with increasing shear rate. The Reynolds number, defined as $\operatorname{Re}=\dot{\gamma} R^{2} / v$ where $R$ is the domain size and $v$ is the kinematic viscosity, varied between 260 and 2300 in the three-dimensional LB simulations [84]. Perhaps counter-intuitively the lowest Re corresponded to the highest shear rate. In this work we will show the influence of a different mechanism, namely one where (thermal) diffusive growth of domains is counterbalanced by convection with the flow. Suppression of domain growth at high shear rates by this mechanism is relevant to phase separating colloidal systems in, e.g., micro-devices. This diffusion - convection mechanism is not active in the quoted LB simulations because thermal diffusion is not included in the model. Another difference is that the LB simulations have focused on the bulk behaviour of phase separating systems under planar shear. In this chapter we take the opposite stand and focus on the influence of confining walls, and the curvature of these walls. We think this is interesting because even without shear, walls can structure and orient the phase separating domains. The precise orientation of the fully phase separated domains will depend on the relative values of fluid-fluid and fluidwall surface tensions. What happens when such systems are sheared, is an open question we wish to address.

In this work we study phase separation of a 50:50 (by volume) fluid mixture in a three dimensional Taylor-Couette geometry (two concentric cylinders), both with and without shear 
flow. We restrict ourselves to reporting interesting observations. We will show that for low and moderate shear rates the final phase separated systems have a banded structure with stacks oriented in a direction that depends on the inner and outer radius of the Couette cell and the applied shear rate. We will also show that at high shear rates complex patterns emerge because the (thermal) diffusive growth of domains is counterbalanced by convection with the flow. This is accompanied by deviations from a homogeneous flow profile. The chapter is organized as follows: in section 3.2 we present the simulation details. In section 3.3 we discuss the results obtained from different configurations and shear rates. In the final section we present the conclusions.

\subsection{Simulation set-up}

In our simulations, the interactions between like particles are based on the Lennard-Jones potential,

$$
U_{L J}\left(r_{i j}\right)=4 \varepsilon\left[\left(\frac{\sigma}{r_{i j}}\right)^{12}-\left(\frac{\sigma}{r_{i j}}\right)^{6}\right]
$$

where $\varepsilon$ and $\sigma$ are the strength and range respectively and $r_{i j}$ is the distance between particles $i$ and $j$. Both the potential and the derived force are smoothly truncated at the cut-off distance $2.5 \sigma$, to eliminate discontinuities at the latter distance. Unlike particles interact by the purely repulsive WCA potential, defined by

$$
U_{W C A}(r)=\left\{\begin{array}{cc}
4 \varepsilon\left[\left(\frac{\sigma}{r}\right)^{12}-\left(\frac{\sigma}{r}\right)^{6}+\frac{1}{4}\right] & \left(r \leq 2^{1 / 6} \sigma\right) \\
0 & \left(r>2^{1 / 6} \sigma\right)
\end{array}\right.
$$

In our simulations, Newton's equation of motion are integrated numerically using the Verlet leap-frog algorithm with a time step $\Delta t=0.002 \tau$ for low shear rate runs, and $\Delta t=0.0005 \tau$ for higher shear rates, where $\tau=\sqrt{\left(m \sigma^{2} / \varepsilon\right)}$ and $m$ is the mass of a particle. The temperature used in the simulations is $k_{B} T=1.0 \varepsilon$, where $k_{B}$ is Boltzmann's constant. To dissipate the energy released by the phase separating system and shear, we use a thermostat. Many thermostats interfere with the creation of hydrodynamics flow fields, and therefore are not suited for the study of the later stages of phase separation. We have used the friction and random forces of dissipative particle dynamics (DPD) $[29,35]$ to thermostat the system, both because 
of its conservation of local momentum, which is also the basis of the Navier-Stokes equation, and because of its avoidance of a profile bias in boundary driven non-equilibrium simulations of shear flow [80]. In DPD the friction force $\mathbf{F}_{\text {fric }}$ and random force $\mathbf{F}_{\text {ran }}$ between a pair of particles separated by a distance $r$ within the cutoff distance $r_{c}$ is given by

$$
\begin{aligned}
\mathbf{F}_{\text {fric }} & =-\frac{\kappa 2}{2 k_{B} T}\left(1-\frac{r}{r_{c}}\right)^{2}(\hat{\mathbf{r}} \cdot \Delta \mathbf{v}) \hat{\mathbf{r}} \\
\mathbf{F}_{\text {ran }} & =\frac{\kappa}{\sqrt{\Delta t}}\left(1-\frac{r}{r_{c}}\right) \zeta \hat{\mathbf{r}}
\end{aligned}
$$

where $\kappa$ is the strength of the friction constant, which we set equal to $3 \varepsilon \tau^{1 / 2} \sigma^{-1}$ in this work, $\hat{\mathbf{r}}$ is the unit vector in the direction of the line joining the two particles, $\Delta \mathbf{v}$ is the velocity difference between the particle pair, and $\zeta$ is a random number with zero mean and unit variance.

To see the effect of confinement and curvature, we simulate a Taylor-Couette geometry with different radii for the inner and outer (concentric) cylinders. In particular, in one of three sets we fix the inner radius to $R_{1}=40 \sigma$ and vary the outer radius between $R_{2}=55 \sigma$ and $80 \sigma$, so that the inner to outer radius ratio is $R_{1} / R_{2}=0.5$ to 0.73 . In the second set we use an inner radius of $R_{1}=25 \sigma$ and an outer radius of $R_{2}=65,70 \sigma\left(R_{1} / R_{2}=0.36\right.$ to 0.38$)$. For the third set we use $R_{1}=15 \sigma$ and $R_{2}=60,65 \sigma\left(R_{1} / R_{2}=0.23\right.$ to 0.25$)$. The density in all these simulations is fixed at $\rho=0.7 \sigma^{-3}$. When changing the outer radius $R_{2}$, the height $Z$ of the box is changed accordingly in order to keep the total volume constant.

To keep the system size manageable we simulate a periodic system with an overall angle of $\Theta_{t}=\pi / 3$, as schematically shown in Fig. 5.1. The total number of particles is 30,000 for simulations starting from a banded state, and 60,000 for simulations starting from randomly mixed configurations. We apply shear by rotating the inner wall with an angular velocity $\omega$ ranging from $0.001 \tau^{-1}$ to $0.1 \tau^{-1}$. Since we simulate only part of a Couette cell, the periodic boundary conditions need to be modified accordingly. Periodic boundary conditions in the $z$ direction are applied as usual. In order to impose periodic boundary conditions in the $\theta$ direction, both for calculating forces between particles on either side of the boundaries and for replacing a particle from one boundary to the other, it is necessary to rotate force and velocity vectors. Specifically, to calculate the velocity $\mathbf{v}$ / of an image particle in the periodic box directly following the central box in the positive $\theta$ direction, it must be calculated from 


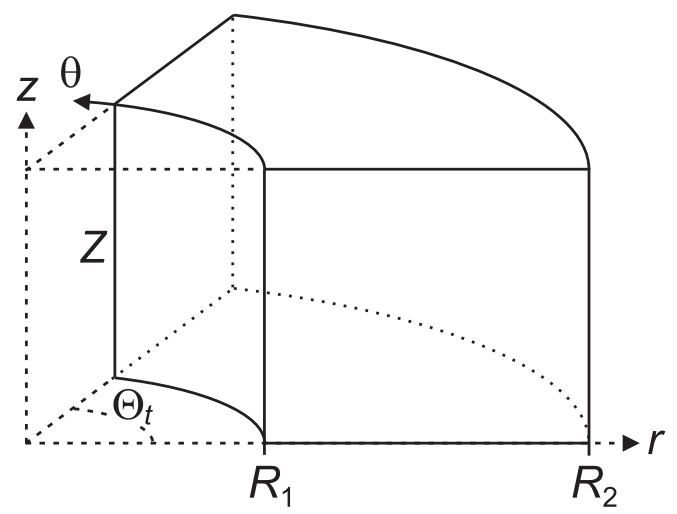

Figure 3.1: Simulated Taylor-Couette cell geometry. Stick boundary walls are present at $r=R_{1}$ and $r=R_{2}$. In the sheared simulations the inner wall is rotating. Periodic boundaries are applied in the flow direction at $\theta=0$ and $\theta=\Theta_{t}$ and in the vorticity direction at $z=0$ and $z=Z$.

the velocity $\mathbf{v}$ of the original particle in the central box by a rotation

$$
\left(\begin{array}{c}
v_{x^{\prime}} \\
v_{y^{\prime}} \\
v_{z^{\prime}}
\end{array}\right)=\left(\begin{array}{ccc}
\cos \left(\Theta_{t}\right) & -\sin \left(\Theta_{t}\right) & 0 \\
\sin \left(\Theta_{t}\right) & \cos \left(\Theta_{t}\right) & 0 \\
0 & 0 & 1
\end{array}\right)\left(\begin{array}{l}
v_{x} \\
v_{y} \\
v_{z}
\end{array}\right) .
$$

A similar rotation applies, with $\Theta_{t}$ substituted by $-\Theta_{t}$, in case of an image particle in the periodic box preceding the central box in the $\theta$ direction.

In the $r$ direction a wall is present at $R_{1}$ and $R_{2}$. A bounce back rule (inversion of the velocity $\mathbf{v} \mapsto-\mathbf{v}$ ) is applied to any particle colliding with the wall. For moving walls, this bounce back rule is relative to a frame co-moving with the wall. Despite the bounce back rule, without additional measures, we found a small amount of slip at the walls, especially at the higher shear rates. To make sure that the shear is applied properly and there is no slip at the wall, all particles within one $\sigma$ distance from the walls are given a new velocity from a Maxwell-Boltzmann distribution, whose average is shifted in the $\theta$ direction to match the velocity of the wall. In this way walls provide stick boundary conditions and at the same time act as a thermostat. 


\subsection{Results and discussion}

We perform three different types of (computer) experiments in our Couette geometry. (i) In the first series of experiments we start with randomly mixed configurations and study phase separation without shear. (ii) In the second we start from fully phase separated systems, under various geometrical conditions, and then suddenly start shear. (iii) In the third series of experiments we start from randomly mixed configurations and then analyze the effect of the onset of shear.

\subsubsection{No shear}

In the first series of experiments we do not apply shear. In this case simple free energy calculations, as given in Appendix 5.5, suffice to predict the final equilibrium states. For example, when fixing the inner radius, varying the outer radius at constant volume changes the relative free energy between different final equilibrium states. In Fig. 3.2 we have plotted free energies for various configurations defined below. We have simulated geometries with inner radius $R_{1}=40 \sigma$ and outer radius from the set of $R_{2}=55,60,65,75,80 \sigma$. The outer radii were carefully chosen to ensure different final configurations.

For the narrowest configuration, with outer radius $R_{2}=55 \sigma$, the equilibrium configuration predicted by free energy calculations consists of two separated phases, stacked along the $z$ direction (here and in the following we call these $z$ stacks). This is also observed in our simulations, see Fig. 3.3. For a slightly less narrow configuration, when the outer radius is $R_{2}=60 \sigma$, the predicted equilibrium configuration consists of stacks with their normal in the $\theta$ direction ( $\theta$ stacks), which is verified in our simulations as well. The next geometry, $R_{2}=65 \sigma$, shows an interesting pattern. According to the free energy calculations the $\theta$ stack still has the lowest free energy, but in our simulation a cylindrical drop forms at the outer radius, see Fig. 3.3. During the course of our simulation it remains like this.

Free energy calculations which also consider the cylindrical drop suggest that this is a metastable state with a free energy very close to that of the $\theta$ stacks. The system is therefore able to stay in this configuration for a prolonged time. We expect that $\theta$ stacks will eventually form, but our equilibrium simulations are not long enough to observe this transition. The next two systems also show interesting behavior. Theoretically, $R_{2}=75 \sigma$ corresponds to a final 


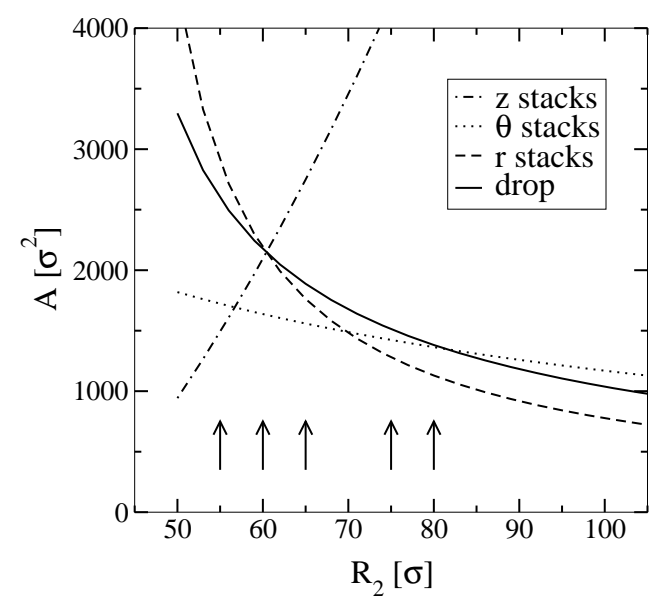

Figure 3.2: Interfacial area between the $A$ and $B$ phases for the case $R_{1}=40 \sigma$ and a fixed volume of $42.857 \sigma^{3}$ (30.000 particles). The arrows indicate the values of $R_{2}$ studied here. We consider the cases of $r$-stacks, $\theta$-stacks and z-stacks, as well as a cylindrical drop at the outer wall. For equally wetting liquids, the configuration of lowest interfacial area is the configuration of lowest free energy.

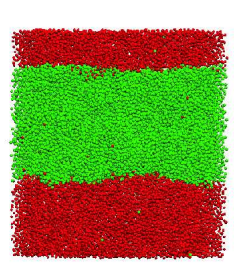

$R_{2}=55$

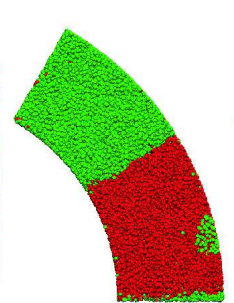

$R_{2}=60$

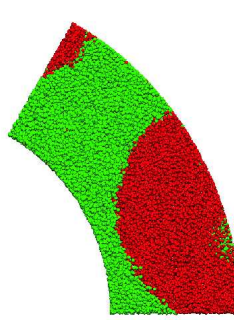

$R_{2}=65$

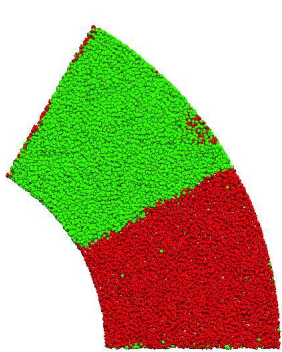

$R_{2}=75$

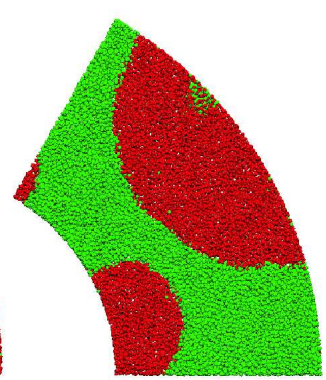

$R_{2}=80$

Figure 3.3: Phase separated configurations for $R_{1}=40 \sigma$ and various $R_{2}$ at a fixed volume of $42.857 \sigma^{3}$ (30.000 particles). For $R_{2}=55 \sigma$ we look from the axis of the cylinder towards the inner wall in the $z-\theta$ plane. For all other values of $R_{2}$ we show the top view $(r-\theta$ plane). 


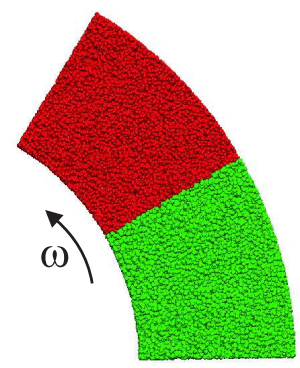

$\omega t=0$

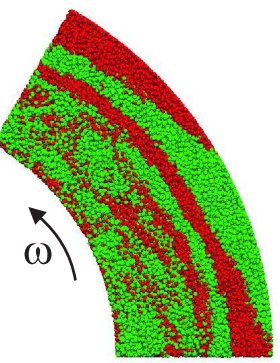

$\omega t=10$

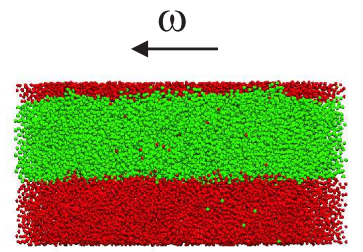

$\omega t=100$

Figure 3.4: Evolution of a sheared system containing pre-assembled $\theta$-stacks. In this particular case $R_{1}=40, R_{2}=65 \sigma$, and $\omega=0.1 \tau^{-1}$. The first two figures are top views $(r-\theta$ plane), in the last figure we look from the inside wall in the $z-\theta$ plane.

equilibrium state of two concentric liquid cylinders ( $r$ stacks in our language). It appears to be very difficult for the liquid in absence of shear to have a stable curved interface and the phase separation observed is a stack in the $\theta$ direction, which is the next lowest free energy configuration. For the last system $\left(R_{2}=80 \sigma\right)$ it is clear from Fig. 3.2 that again $r$ stacks (concentric liquid cylinders) should form the most stable configuration, but in the simulation the system is trapped in a metastable state of multiple cylindrical droplets on either walls. These drops keep moving but within the course of our simulation they do not merge to a final state. In summary, for the narrow systems we observe full phase separation in agreement with free energy calculations, while for the wider systems we observe configurations that are stuck in metastable states, which are often the next lowest free energy configurations.

\subsubsection{With shear, starting from phase separated systems}

Before studying the influence of shear on the kinetics of phase separation we investigate what shear does with the equilibrium configurations going with particular geometries. We focus on three different geometries with $R_{1}=40 \sigma$ and $R_{2}=55,65,75 \sigma$. As we have seen above, without shear the first corresponds to a final equilibrium of $z$ stacks, the second to $\theta$ stacks and the third to $r$ stacks. For each of these geometries we start with three different initial conditions, namely stacks in the $r, \theta$ or $z$ direction. Obviously, only one of them corresponds 


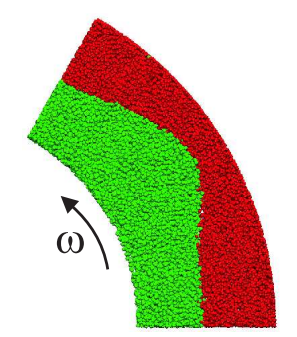

Figure 3.5: For $r$-stacks under sufficiently high shear a single heap emerges which moves in the $\theta$ direction. In this case $R_{1}=40 \sigma, R_{2}=65 \sigma$ and $\omega=0.1 \tau^{-1}$.

to the equilibrium situation without shear. We apply two different shear rates by rotating the inner wall with an angular velocity of $\omega=0.01$ or $0.1 \mathrm{rad} \tau^{-1}$.

One feature which is common to both $z$ (vorticity) and $r$ (gradient) stacks is the extreme slowness of transitions from one configuration to another. Starting from a stack in the $z$ direction, the system continues to be $z$-stacked for the duration of the simulation, at both shear rates studied. This includes geometries which would not prefer to form $z$-stacks at rest, nor if we would start from a randomly mixed simulation. Analogous results are obtained starting from stacks in the $r$ direction. Although we are not able yet to quantify the effect, it can qualitatively be explained on the basis of existing free energy barriers. Thermal fluctuations and shear are not enough to overcome the free energy barrier in the time simulated.

It is impossible for $\theta$ stacks to persist when the inner wall is rotating, so such configurations must quickly disappear and change to other configurations. Fig. 3.4 shows that if we start from a stack in the $\theta$ (flow) direction, quickly streaks are elongated along the flow direction, which are then pushed in the outward direction. These moving bands merge and then result in stacks in the $z$ direction. These $z$ stacks are stable for all shear rates applied in our simulations. Notice that the view towards the axis will be one of bands stacked along the vorticity direction. In case of $r$ stacks an interesting phenomenon occurs in Couette shear flow: a single interfacial crest develops at the interface which moves like a soliton in the flow direction (we use the word soliton-like, because we do not imply the rather specific meaning of a soliton in the context of non-linear, dispersive waves, but rather its characteristics of a localised "heap" that is moving in space without changing shape). At low shear rates this 
interfacial heap is barely visible but at higher shear rates it is very prominent, as shown in Fig. 3.5. The curvature of the wall is an essential ingredient of this phenomenon because no heap is observed in simulations containing the same phase-separated fluid in a planar shear geometry with the same gap-width and subjected to the same shear rates. We will now analyse the angular velocity of the heap. Let the radial coordinate of the interface for each value of $\theta$ and $t$ be given by $R(\theta, t)$. The soliton-like character may then be expressed as

$$
R(\theta, t)=R(\theta-\Omega t, 0)
$$

where $\Omega$ is the angular velocity of the heap. To measure this velocity we must be careful not to measure the velocities of the individual particles, but only their collective effect on the interfacial perturbation. To this end we define

$$
\rho_{m}(t)=\sum_{i=1}^{N} B_{i} \cos \left(m \theta_{i}(t)\right),
$$

where $B_{i}= \pm 1$ depending on the particle type and $\theta_{i}$ is the polar angle of particle $i$. We choose an angular mode number $m=6$ or 12 to comply with our periodic setup. It is not difficult to show (see Appendix 5.5) that in the continuum limit

$$
\rho_{m}(t)=X \cos (m \Omega t)+Y \sin (m \Omega t) .
$$

The time correlation of $\rho$ is then calculated as

$$
\left\langle\rho_{m}(0) \rho_{m}(t)\right\rangle=\frac{1}{2}\left(X^{2}+Y^{2}\right) \cos (m \Omega t),
$$

where $\langle\ldots\rangle$ denotes an average over many time origins. An example is shown in Fig. 3.6. Despite the fact that the interfacial heap is barely visible at lower shear rates, the correlation function Eq. (3.8) still does a good job at measuring its angular velocity. Fig. 3.7 shows the measured angular velocity of the interfacial heap versus the angular velocity $d \theta_{H S} / d t$ of a homogeneous fluid at the average radial position $R_{a v}=\sqrt{\left(R_{1}^{2}+R_{2}^{2}\right) / 2}$. To collect sufficient points for this figure we have performed additional simulations at $\omega=0.02 \tau^{-1}$ and $0.05 \tau^{-1}$. As expected, for low shear rates the angular velocity of the interface is the same as that of a homogeneous liquid at $r=R_{a v}$. But at higher shear rates the angular velocity of the interfacial heap decreases appreciably relative to the convection value. It is easily seen that any acceptable alternative definition of the horizontal axis in this plot will only increase the 


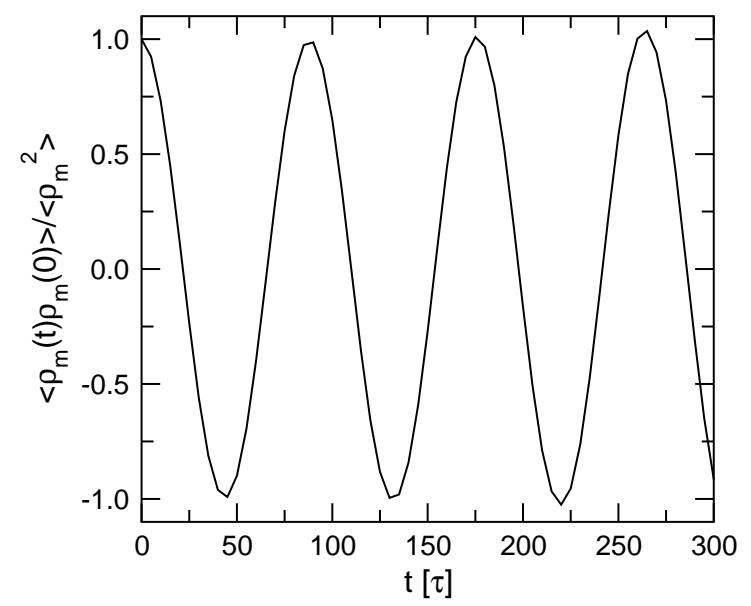

Figure 3.6: Time-correlation function of the angular difference in A and B-type density, see Eqs. (3.6) and (3.8) in the main text, for the system in Fig. 3.5. From this we can calculate the angular velocity of the heap in a system containing r-type stacks.

deviation from the $y=x$ line. Volume-conservation dictates that the same amount of A particles are displaced to $r$ values beyond $R_{a v}$ as there are B particles displaced to $r$ values below $R_{a v}$. Because of the cylindrical geometry, the distance between $R_{a v}$ and the lowest value of $r$ of the interface will be larger than the distance between $R_{a v}$ and the highest value of $r$ of the interface. If anything, a naive consideration of the effect of the moving inner wall would predict a higher average angular velocity. The lower velocity is probably caused by the additional hydrodynamic friction in the narrow region between the extremes of the interface and the inner and outer walls. A detailed analysis of this interfacial heap will be a topic of future research.

\subsubsection{With shear, starting from randomly mixed systems}

Finally, we focus on initially randomly mixed configurations, which are simultaneously phase separating and subjected to shear. In our previous work [89] we studied phase separation from a randomly mixed configuration in bulk without shear. We showed that the length scale $R$ characterising the phase separation initially grows in time according to $R(t) \propto t^{1 / 3}$, 


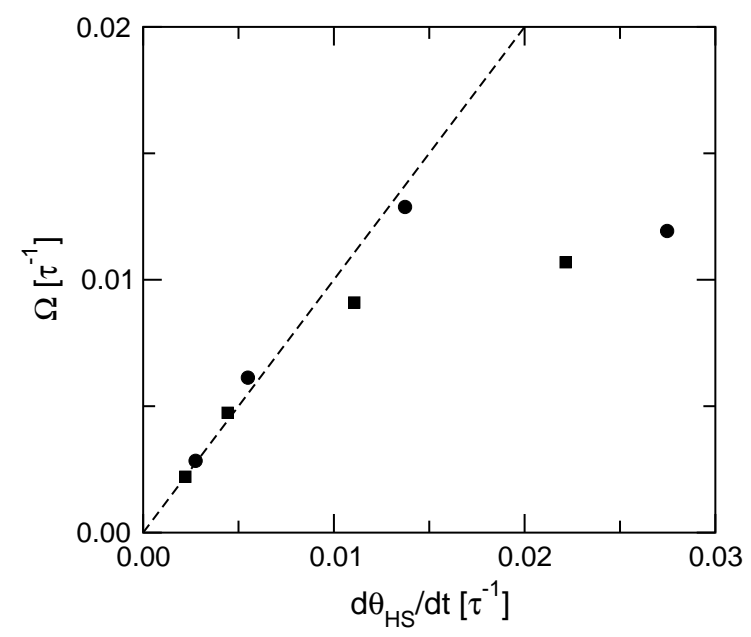

Figure 3.7: Measured angular velocity of the heap versus the angular velocity of a homogeneous fluid at the average position of the interface. The dashed line indicates where both velocities are equal. Circles are measurements from a system with $R_{1}=40 \sigma$ and $R_{2}=65 \sigma$. Squares are measurements from $R_{1}=40 \sigma$ and $R_{2}=75 \sigma$.

corresponding to a diffusion limited growth of the domains. After a time $\tau_{d v} \approx 25 \tau$ the scaling law changes to a surface tension driven growth dominated by viscous forces: $R(t) \propto t^{0.8}$ to $t^{1}$. These simulations concerned the same model as in the current study, only then bulk periodic boundary conditions were used, whereas now we focus on the influence of confinement and curvature combined with shear.

The confinement and curvature will, as before, lead to a preference of a specific stacked structure without shear. At low shear rates this structure may remain, but other stacked structures may become more stable at higher shear rates. For example, in sheared diblock copolymer melts a transition is observed from perpendicular to parallel orientations $[47,51,63]$. At very high shear rates no stable stacked structure may form at all. In particular with reference to the latter case, we expect that there will be a critical shear rate $\dot{\gamma}_{d v} \approx 1 / \tau_{d v}$ separating a regime of stable stacked configurations from a regime of unstable configurations. The reason is the following: Imagine two domains of size $R$ with their centres displaced by a distance of order $R$ in the gradient direction. For shear rates $\dot{\gamma} \ll \dot{\gamma}_{d v}$ the shear rate is so low that viscous, 


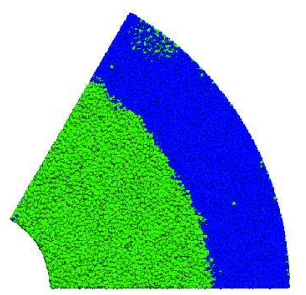

$\omega=0.001$

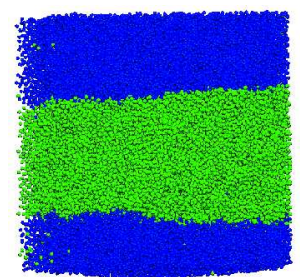

$\omega=0.01$

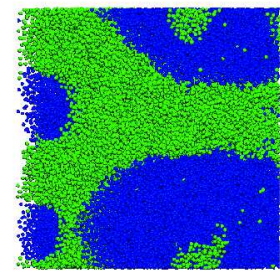

$\omega=0.1$

Figure 3.8: Configurations of the system with $R_{1}=15 \sigma, R_{2}=60 \sigma$ at various angular velocities of the inner wall. The first figure is a top view ( $r$ - $\theta$ plane) at $\omega=0.001 \tau^{-1}$, showing the formation of steady r-stacks. The second figure is a front view ( $r$-z plane) at $\omega=0.01 \tau^{-1}$, showing the formation of steady z-stacks. The third figure is also a front view at $\omega=0.1 \tau^{-1}$, showing one cross-section of the complex patterns that emerge and are kinetically trapped.

surface tension driven growth has begun before the affine deformation has had any chance to separate and deform the relative position of these two domains. For shear rates $\dot{\gamma} \gg \dot{\gamma}_{d v}$, on the other hand, the shear flow will interfere with the diffusive growth of the domains: the two domains will have been separated and deformed before they have had any chance to diffuse towards each other.

To test the above hypotheses, we performed sheared simulations in four different geometries, two simulations with $R_{1}=25 \sigma$ and $R_{2}=65$ and $70 \sigma$ respectively, and another two with $R_{1}=15 \sigma$ and $R_{2}=60$ and $65 \sigma$ respectively. Note that, ignoring the curvature effect for the moment, the average shear rate in the slit is approximately equal to $\omega R_{1} /\left(R_{2}-R_{1}\right)$, i.e. about equal to $\omega$ in the systems studied.

All configurations were thoroughly equilibrated and mixed, by means of zero shear rate runs with interactions between unlike particles equal to those between like particles, Eq. (3.1). After equilibration, shear was imposed by rotating the inner wall with an angular velocity $\omega=0.001,0.01$ or $0.1 \tau^{-1}$. The resulting flow profiles of these homogeneous systems agreed well with theoretical predictions (not shown). Finally, the repulsion between unlike particles was turned on, Eq. (3.2), and the mixture started to phase separate. Free energy calculations suggest that in all these systems $r$-stacks are the most stable configuration without shear. Indeed we found that at low shear rates, $\omega=0.001 \tau^{-1}, r$-stacks are formed and remain 


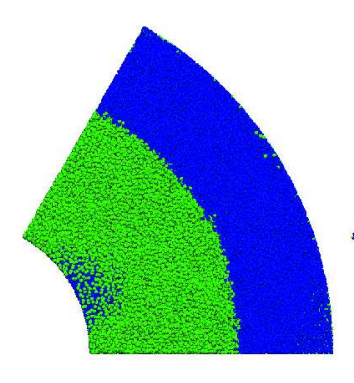

$\omega=0.001$

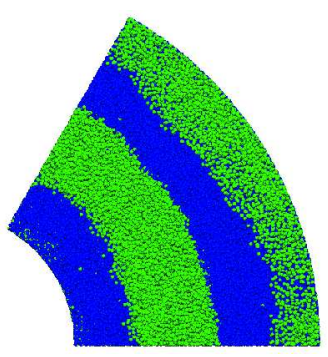

$\omega=0.01$

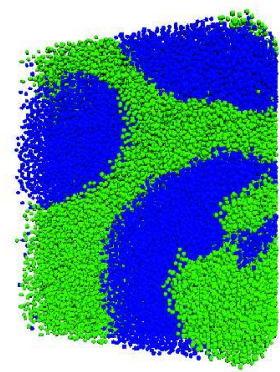

$\omega=0.1$

Figure 3.9: Configurations of the system with $R_{1}=25 \sigma, R_{2}=70 \sigma$ at various angular velocities of the inner wall. The first figure is a top view ( $r-\theta$ plane) at $\omega=0.001 \tau^{-1}$, showing the formation of steady r-stacks. The second figure is a top view at $\omega=0.01 \tau^{-1}$, showing a probably intermediate state of multiple (sandwiched) r-stacks. The third figure is a front view ( $r$-z plane), in perspective and slightly tilted around the $z$-axis, at $\omega=0.1 \tau^{-1}$, showing the complex patterns that emerge.

stable for the duration of the simulation, see Figs. 3.8 and 3.9 (leftmost figures).

When the shear rate was increased to $\omega=0.01 \tau^{-1}$, a transition from steady $r$-stacks to steady $z$-stacks was found for both $R_{1}=15 \sigma, R_{2}=60$ and $R_{1}=15 \sigma, R_{2}=65 \sigma$, see Fig. 3.8 (middle figure). This is analogous to the case of sheared diblock-copolymer melts alluded to above, where a transition is observed from parallel stacks, with their normals in the gradient direction (here $r$ ) to perpendicular stacks, with their normals in the vorticity direction (here z) $[47,51,63]$. For $R_{1}=25 \sigma$ and $R_{2}=65,70$ multiple (sandwiched) $r$-stacks appear at these high shear rates, see Fig. 3.9 (middle figure). This may again be a meta-stable state in which the system is trapped on its way towards a state with just two stacks. It may be very difficult for domains trapped in some metastable state to merge as the only way they can coalesce is by fluctuation of the various interfaces. In all systems with angular velocities $\omega=$ $0.1 \tau^{-1}$ we observe unsteady complex patterns, see Figs. 3.8 and 3.9 (right figures), which do not seem to evolve to any of the stacked states discussed previously. Rather a spongelike, streaked structure forms, in which the system appears to be kinetically trapped. While all other simulations are in the regime $\dot{\gamma} \ll \dot{\gamma}_{d v}=0.04 \tau^{-1}$, those with $\omega=0.1 \tau^{-1}$ all have 
$\dot{\gamma} \geq \dot{\gamma}_{d v}$. When the shear rate is near $\dot{\gamma}_{d v}$ the diffusive growth is prohibited by convection with the flow. This is substantiated by inspecting the velocity profile, see Fig. 3.10. At $\omega=0.1$ the velocity profile deviates from that of a homogeneous liquid. High shear rate boundaries emerge at particular values of $r$ (in this case $r \approx 45 \sigma$ ). Diffusion along the gradient direction becomes ineffective across these high shear boundaries. Within each region separated by

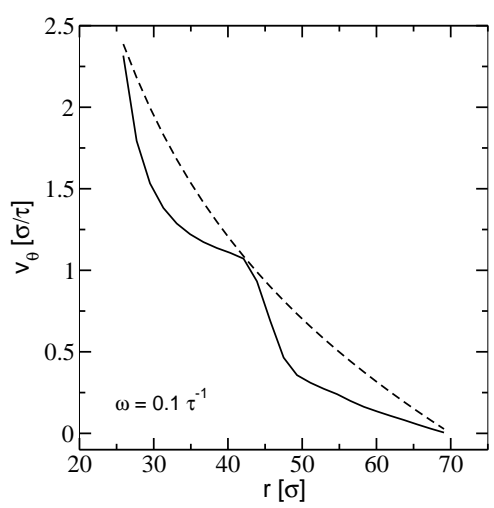

Figure 3.10: Average velocity profile as a function of radial position $r$ in the gap (solid line) for $R_{1}=25 \sigma, R_{2}=70 \sigma$ and $\omega=0.1 \tau^{-1}$ (right figure in Fig. 3.9). The profile for $a$ homogeneous fluid is given by the dashed line. Note the large shear rate at $r \approx 45 \sigma$.

these boundaries, the effective shear rate is lower, and the system is able to form more-or-less stacked states. Indeed, if one is pressed on identifying stacked structures, in Fig. 3.9 (right figure) there appears to be an inner 'z-stack' and an outer 'z-stack' (mixed with an 'r-stack'). The high shear boundary allows the outer stack to be shifted relative to the inner one. A horizontal slice would therefore give the appearance of 'r-stacks'.

It is interesting to compare these results with those of Lattice Boltzmann (LB) simulations $[31,82,84]$ published recently. As already mentioned in the introduction, thermal diffusion is absent from these LB simulations. Moreover, these works focused on the effect of planar shear flow on phase separation in bulk, whereas ours focuses on the effect of confining walls and curvature. In the LB simulations, every effort was undertaken to avoid finite size effects. Finite size effects typically result in quasi-laminar stripe domains which connect after one or more circuits of the periodic boundary conditions [82]. The stacked domains observed in our 
work at lower shear rates could be classified as such. In fact, finite size effects are usually so large that it is impossible for MD simulations (of the type we present here) to study phase separation at the scale of the quoted LB simulations.

\subsection{Conclusions}

We have studied the influence of confinement, curvature and shear on the phase separation in a 50:50 fluid mixture. When starting from stacked configurations either in the gradient or in the vorticity direction, we observe that these pre-arranged configurations are retained for a very long time, suggesting the presence of large free energy barriers to evolve, if at all, to other configurations. The stacks with their normal in the gradient direction show interesting interfacial perturbations, whose angular velocities decrease relative to the expected angular velocity of a homogeneous fluid at the average interfacial position.

Starting from a randomly mixed configuration, the phase separation evolves towards steady stacks at moderate shear rates. The orientation of these stacks appears, in some cases, to depend on the applied shear rate. At high shear rates we do not observe stacks but complex streaked patterns, which are separated by high shear rate boundaries. The transition from steady stacks to complex patterns takes place at a shear rate equal to the inverse of the cross-over time from diffusive to viscous growth of phase-separated domains, as measured in equilibrium simulations. At these shear rates diffusive growth is prohibited convection with the flow.

For small molecules the time to diffuse over a length scale comparable to the size of the molecule is very short, of the order of several picoseconds. The critical shear rate, alluded to above, will in that case be extremely large, probably much larger than can be achieved in experiment. Colloidal particles of size 0.1 to $1 \mu \mathrm{m}$, however, diffuse over their own diameter on time scales of milliseconds to seconds. We therefore believe that our results are relevant to for example microfluidic systems containing such colloidal particles. Certainly, bends and corners in microfluidic devices often have the same curvature as those studied here. We may even envisage the construction of a microscopic Taylor-Couette flow cell, with which our results could be validated. We leave this as a challenge to the experimentalists. 


\section{Appendix A: Interfacial free energy in a Taylor-Couette geometry}

In this Appendix we will calculate the interfacial free energy of two fully phase-separated liquids $\mathrm{A}$ and $\mathrm{B}$ in a Taylor-Couette geometry with inner radius $R_{1}$, outer radius $R_{2}$, height $Z$ and an overall angle $\Theta_{t}$ (see Fig. 5.1). The latter angle is relevant for our periodic setup; for a real Taylor-Couette geometry of course $\Theta_{t}=2 \pi$. We assume a 50:50 (by volume) mixture of the two liquids. We will first treat the case where both liquids are equally wetting the walls, as is the case in our simulations. Experimentally, it is often difficult to attain an equal wettability condition for two different liquids. For completeness, we therefore also treat the case of liquids of different wettability.

\section{Free energy calculation for equally wetting liquids}

When the surface tension with the wall is equal for both liquids, the configuration of lowest interfacial free energy is simply the configuration of lowest interfacial area $A_{A B}$ between liquids A and B. Also, the contact angle that the liquid-liquid interface makes with a wall (if it makes contact with a wall at all) is 90 degrees. The combination of these two facts leads to the prediction of relatively simple configurations for fully phase separated systems in a Couette cell. Let us explore the possibilities.

First, consider the case of two concentric liquid cylindrical shells. Since the normal of the interface points in the $r$ direction, we refer to these as $r$ stacks. The area $A_{A B}$ between the two liquids simply is

$$
A_{A B}=Z \Theta_{t} \sqrt{\frac{R_{1}{ }^{2}+R_{2}^{2}}{2}} .
$$

Second, we may think of two stacks with their interfaces in the $r$ - $z$ plane, so-called $\theta$ stacks. In the case of periodic boundaries in the $z$ direction there are two liquid interfaces per periodic cell, the total area of which is

$$
A_{A B}=2\left(R_{2}-R_{1}\right) Z
$$

Third, two layers may form in the $r$ - $\theta$ plane, so-called $z$ stacks. Again there are two interfaces, 
the total area of which is

$$
A_{A B}=\Theta_{t}\left(R_{2}{ }^{2}-R_{1}^{2}\right)
$$

Finally, consider a semi-cylindrical drop attached to the outer wall. In this case the total interfacial area is given by

$$
A_{A B}=2 r Z \arctan \frac{R_{2} / d}{\sqrt{1-R_{2}^{2} / d^{2}}} .
$$

Here $d^{2}=R_{2}{ }^{2}+r^{2}$, where $r$ is the radius of curvature of the cylindrical drop. It can be found by the condition that the drop must be of volume $Z \Theta_{t}\left(R_{2}^{2}-R_{1}^{2}\right) / 4$, i.e. half the volume of the couette cell, and that the interface must touch the wall at right angles.

\section{Interfacial free energy calculation for liquids with different wetta- bility}

In most practical cases the surface tension $\gamma_{A W}$ between liquid $\mathrm{A}$ and the wall is different from the surface tension $\gamma_{B W}$ between liquid B and the wall. In that case the lowest interfacial free energy corresponds to a more complex configuration, and the contact angle with the wall is no longer 90 degrees. The shape of the interface will depend on the relative values of the wall interfacial tensions $\gamma_{A W}, \gamma_{B W}$ and the interfacial tension $\gamma_{A B}$ between both liquids. For not too widely different values of the surface tensions, we again get different layered structures whose normals are primarily oriented in the $r, \theta$ or $z$ directions.

\section{The case of $r$ stacks}

The case of two concentric cylindrical shells is still a possible solution, and the simplest of the three. If we assume $\gamma_{A W}>\gamma_{B W}$ then liquid A will reside at the inner wall and liquid $\mathrm{B}$ at the outer wall. The interfacial free energy is then given by

$$
A=Z \Theta_{t}\left[R_{1} \gamma_{A W}+R_{2} \gamma_{B W}+\sqrt{\frac{R_{1}{ }^{2}+R_{2}^{2}}{2}} \gamma_{A B}\right] .
$$




\section{The case of $\theta$ stacks}

The equilibrium interface between two liquid phases in a Couette geometry can be calculated using the principle of free energy minimization. In case of $\theta$ stacks, we choose our coordinates such that fluid $\mathrm{A}$ is centered near the plane $\theta=0$. The interface with fluid $\mathrm{B}$ is then described by a function $\theta(r)$, in cylindrical coordinates (see Fig. 5.1). For reasons of symmetry, we assume that the interface is independent of height $z$ and that another AB interface (within our periodic cell) is situated at $\Theta_{t}-\theta(r)$.

Since the total interfacial free energy must be minimal, under the constraint of constant volume of fluid A, the Lagrangian to be minimized is

$$
\begin{aligned}
L=\int_{R_{1}}^{R_{2}} d r & {\left[\gamma_{A B} \sqrt{1+r^{2} \dot{\theta}^{2}}-\lambda^{\prime} r \theta\right]+\gamma_{A W}\left[\theta\left(R_{1}\right) R_{1}+\theta\left(R_{2}\right) R_{2}\right] } \\
+\gamma_{B W}\left[\left(\Theta_{t} / 2-\theta\left(R_{1}\right)\right) R_{1}+\right. & \left.\left(\Theta_{t} / 2-\theta\left(R_{2}\right)\right) R_{2}\right]
\end{aligned}
$$

where $\dot{\theta}=d \theta / d r$ and $\lambda^{\prime}$ is a Lagrange multiplier to constrain the volume, i.e. the area in the $r-\theta$ plane, of fluid A. Applying a small perturbation

$$
\theta(r) \rightarrow \theta(r)+\eta(r)
$$

the Lagrangian will change like

$$
\delta L=\int_{R_{1}}^{R_{2}} d r\left[\gamma_{A B} \frac{r^{2} \dot{\theta}}{\sqrt{1+r^{2} \dot{\theta}^{2}}} \dot{\eta}-\lambda^{\prime} r \eta\right]+\bar{\gamma}_{W}\left[\eta\left(R_{1}\right) R_{1}+\eta\left(R_{2}\right) R_{2}\right],
$$

where $\dot{\eta}=d \eta / d r$ and $\bar{\gamma}_{W}$ is $\gamma_{A W}-\gamma_{B W}$. Partial integration yields

$$
\begin{aligned}
\delta L= & {\left[\frac{R_{2}^{2} \dot{\theta}\left(R_{2}\right)}{\sqrt{1+R_{2}^{2} \dot{\theta}\left(R_{2}\right)^{2}}} \eta\left(R_{2}\right)-\frac{R_{1}^{2} \dot{\theta}\left(R_{1}\right)}{\sqrt{1+R_{1}^{2} \dot{\theta}\left(R_{1}\right)^{2}}} \eta\left(R_{2}\right)\right] \gamma_{A B} } \\
& -\int_{R_{1}}^{R_{2}} d r\left[\gamma_{A B} \frac{d}{d r} \frac{r^{2} \dot{\theta}}{\sqrt{1+r^{2} \dot{\theta}^{2}}}-\lambda^{\prime} r\right] \eta+\bar{\gamma}_{W}\left[\eta\left(R_{1}\right) R_{1}+\eta\left(R_{2}\right) R_{2}\right] .
\end{aligned}
$$

Putting $\delta L$ equal to zero for any small perturbation $\eta(r)$ gives rise to three equations:

$$
\begin{aligned}
& \frac{d}{d r} \frac{r^{2} \dot{\theta}}{\sqrt{1+r^{2} \dot{\theta}^{2}}}=\lambda r, \\
& \frac{R_{1} \dot{\theta}\left(R_{1}\right)}{\sqrt{1+R_{1}^{2} \dot{\theta}\left(R_{1}\right)^{2}}}=\frac{\overline{\gamma_{W}}}{\gamma_{A B}} \\
& \frac{R_{2} \dot{\theta}\left(R_{2}\right)}{\sqrt{1+R_{2}^{2} \dot{\theta}\left(R_{2}\right)^{2}}}=-\frac{\overline{\gamma_{W}}}{\gamma_{A B}},
\end{aligned}
$$


where $\lambda=\lambda^{\prime} / \gamma_{A B}$. Solving for $\dot{\theta}$ we find

$$
\begin{aligned}
\dot{\theta}^{2} & =\frac{\left(C+\frac{1}{2} \lambda r^{2}\right)^{2}}{r^{4}-r^{2}\left(C+\frac{1}{2} \lambda r^{2}\right)^{2}}, \\
\theta(r) & =\theta\left(R_{1}\right)+\int_{R_{1}}^{r} d x \frac{C+\frac{1}{2} \lambda x^{2}}{\sqrt{x^{4}-x^{2}\left(C+\frac{1}{2} \lambda x^{2}\right)^{2}}},
\end{aligned}
$$

where $C$ is a constant. From Eq. (A.10) we obtain

$$
R_{1} \dot{\theta}\left(R_{1}\right)=\frac{\bar{\gamma}_{W} / \gamma_{A B}}{\sqrt{1-\left(\bar{\gamma}_{W} / \gamma_{A B}\right)^{2}}},
$$

which must be consistent with Eq. (A.12). Differentiating the latter, multiplying by $R_{1}$, and comparing the result with Eq. (A.13), we find after some rearrangements

$$
\frac{C}{R_{1}}+\frac{1}{2} \lambda R_{1}=\frac{\bar{\gamma}_{W}}{\gamma_{A B}} .
$$

Similarly, from Eq. (A.11) and Eq. (A.12), we obtain

$$
\frac{C}{R_{2}}+\frac{1}{2} \lambda R_{2}=-\frac{\bar{\gamma}_{W}}{\gamma_{A B}} .
$$

Solving for $C$ and $\lambda$ we get

$$
\begin{aligned}
C & =\frac{R_{1} R_{2}}{R_{2}-R_{1}} \frac{\bar{\gamma}_{W}}{\gamma_{A B}} \\
\lambda & =2 \frac{\bar{\gamma}_{W}}{\gamma_{A B}} \frac{1}{R_{1}-R_{2}}
\end{aligned}
$$

The shape of the interface is now known, and only its starting point $\theta\left(R_{1}\right)$ at the wall remains to be determined. This is not difficult, as half the total volume must be occupied by fluid A. A little more algebra yields

$$
\theta\left(R_{1}\right)=\frac{\Theta_{t}}{4}-\int_{R_{1}}^{R_{2}} d r \frac{R_{2}^{2}-r^{2}}{R_{2}^{2}-R_{1}^{2}} \frac{C+\frac{1}{2} \lambda r^{2}}{\sqrt{r^{4}-r^{2}\left(C+\frac{1}{2} \lambda r^{2}\right)^{2}}}
$$

The interfacial free energy, finally, is given by

$$
A=Z\left(\gamma_{A W} L_{A W}+\gamma_{B W} L_{B W}+\gamma_{A B} L_{A B}\right)
$$

with

$$
\begin{aligned}
L_{A W} & =2 R_{1} \theta\left(R_{1}\right)+2 R_{2} \theta\left(R_{2}\right) \\
L_{B W} & =R_{1}\left(\Theta_{t}-2 \theta\left(R_{1}\right)\right)+R_{2}\left(\Theta_{t}-2 \theta\left(R_{2}\right)\right) \\
L_{A B} & =2 \int_{R_{1}}^{R_{2}} d r \sqrt{\frac{1}{1-\left(\frac{C}{r}+\frac{1}{2} \lambda r\right)^{2}}}
\end{aligned}
$$


It is perhaps illuminating to define

$$
R_{1} \dot{\theta}\left(R_{1}\right)=\tan (\pi / 2-\alpha),
$$

where $\alpha$ is the angle between the AB-interface and the inner cylinder. Eq. (A.13) may then be rewritten as Young's equation

$$
\gamma_{A W}-\gamma_{B W}+\gamma_{A B} \cos \alpha=0
$$

\section{The case of $z$ stacks}

For the $z$ stacks the profile can be derived in a similar fashion, in which case we choose our coordinates such that fluid $\mathrm{A}$ is centered around the plane $z=0$. The upper interface with fluid B is then described by a function $z(r)$, again in cylindrical coordinates. For reasons of symmetry we assume that the interface is independent of $\theta$ and that another AB interface (within our periodic cell) is situated at $Z-z(r)$.

Now the Lagrangian to be minimized is

$$
L=\int_{R_{1}}^{R_{2}} d r\left[\gamma_{A B} r \sqrt{1+\dot{z}^{2}}-\lambda^{\prime} r z\right]+\overline{\gamma_{W}}\left[z\left(R_{1}\right) R_{1}+z\left(R_{2}\right) R_{2}\right],
$$

where $\dot{z}=d z / d r$ and again $\lambda^{\prime}$ is a Lagrange multiplier to constrain the volume (now the area in the $r$ - $z$ plane). The resulting profile is given by

$$
z(r)=z\left(R_{1}\right)+\int_{R_{1}}^{r} d x \frac{C+\frac{1}{2} \lambda x^{2}}{\sqrt{x^{2}-\left(C+\frac{1}{2} \lambda x^{2}\right)^{2}}},
$$

where $C$ and $\lambda$ are the same as in Eqs. (A.16) and (A.17). The value of $z\left(R_{1}\right)$ can again be fixed by setting the volume occupied by fluid A equal to half the total volume. This yields

$$
z\left(R_{1}\right)=\frac{Z}{4}-\int_{R_{1}}^{R_{2}} d r \frac{R_{2}^{2}-r^{2}}{R_{2}^{2}-R_{1}^{2}} \frac{C+\frac{1}{2} \lambda r^{2}}{\sqrt{r^{2}-\left(C+\frac{1}{2} \lambda r^{2}\right)^{2}}} .
$$

The interfacial free energy is then given by

$$
\begin{aligned}
A & =\Theta_{t}\left(\gamma_{A W} A_{A W}+\gamma_{B W} A_{B W}+\gamma_{A B} A_{A B}\right) \\
A_{A W} & =2 R_{1} z\left(R_{1}\right)+2 R_{2} z\left(R_{2}\right) \\
A_{B W} & =R_{1}\left(Z-2 z\left(R_{1}\right)\right)+R_{2}\left(Z-2 z\left(R_{2}\right)\right) \\
A_{A B} & =2 \int_{R_{1}}^{R_{2}} d r r \sqrt{\frac{1}{1-\left(\frac{C}{r}+\frac{1}{2} \lambda r\right)^{2}}}
\end{aligned}
$$




\section{Appendix B: Derivation of heap's angular velocity}

The continuum approximation of Eq. (3.7) reads

$$
\begin{aligned}
\rho_{m}(t) & =\rho \int_{0}^{Z} d z \int_{0}^{\pi / 3} d \theta \cos (m \theta) \int_{R_{1}}^{R_{2}} d r r(1-2 H(r-R(\theta, t))) \\
& =\rho Z \int_{0}^{\pi / 3} d \theta \cos (m \theta) R^{2}(\theta, t),
\end{aligned}
$$

where $H(x)$ is Heaviside's function, i.e. $H(x)=0$ for $x<0$ and $H(x)=1$ for $x \geq 0$, and where $m$ is a multiple of six. Using the soliton-like character expressed in Eq. (3.5), we transform this into

$$
\begin{aligned}
\rho_{m}(t)= & \rho Z \int_{0}^{\pi / 3} d \theta \cos (m \theta) R^{2}(\theta-\Omega t, 0) \\
= & \rho Z \int_{-\Omega t}^{\pi / 3-\Omega t} d \theta \cos (m \theta+m \Omega t) R^{2}(\theta, 0) \\
= & \rho Z \cos (m \Omega t) \int_{0}^{\pi / 3} d \theta \cos (m \theta) R^{2}(\theta, 0) \\
& -\rho Z \sin (m \Omega t) \int_{0}^{\pi / 3} d \theta \sin (m \theta) R^{2}(\theta, 0) \\
\equiv & X \cos (m \Omega t)+Y \sin (m \Omega t) .
\end{aligned}
$$

Using this result, we calculate the time correlation function

$$
\begin{aligned}
\left\langle\rho_{m}(t) \rho_{m}(0)\right\rangle & =\frac{1}{T} \int_{0}^{T} d \tau \rho_{m}(t+\tau) \rho_{m}(\tau) \\
& =\frac{1}{2}\left(X^{2}+Y^{2}\right) \cos (m \Omega t)
\end{aligned}
$$

where $T$ is a multiple of $2 \pi /(m \Omega)$. 



\title{
1 Spinodal decomposition in asymmetric binary fluids
}

\begin{abstract}
The spinodal decomposition of quenched polymer/solvent and liquid-crystal/solvent mixtures in a miniature Taylor-Couette cell has been simulated by molecular dynamics (MD). Three stacking motifs, each reflecting the geometry and symmetry of the cell, are most abundant among the fully phase-separated stationary states. At zero or low angular velocity of the inner cylindrical drum, the two segregated domains have a clear preference for the stacking with the lowest free energy and hence the smallest total interfacial tension. For high shear rates the steady state appears to be determined by a minimum dissipation mechanism, i.e. the mixtures are likely to evolve into the stacking demanding the least mechanical power by the rotating wall. The partial slip at the polymer-solvent interfaces then gives rise to a new pattern: a stack of three concentric cylindrical shells with the viscous polymer layer sandwiched between two solvent layers. Neither of these mechanisms can explain all simulation results, as the separating mixture easily becomes kinetically trapped in a long-lived sub-optimal configuration. The phase separation process is observed to proceed faster under shear than in a quiescent mixture.
\end{abstract}

\subsection{Introduction}

Phase separation is a common phenomenon in mixtures of liquids and in liquids dispersed with buoyant bodies, like colloids, polymers or surfactants, where small differences between 
the mutual attractions of the various molecules are capable of separating like molecules from unlike molecules, thus driving the formation of clusters or extended patterns. Phase separation may occur in many industrial processes dealing with compound fluids, like paints, foods and health-care products, where it often has undesired detrimental effects, and shortens the shelf-life of many products [30]. The kinetics of phase separation has therefore attracted the attention of many researchers over the years, and still remains a topic of intense study to date.

A central quantity in the study of spinodal decomposition - the system-wide phase separation immediately following a quench in one of the system parameters - is the evolution of the average domain size $R(t)$ of the emerging patterns or aggregates, with $t$ denoting the time since the quench. Theoretical models of the growth process, like the 'model H' for fluid-fluid phase separation, combine a Ginzburg-Landau-type expression for the free energy as a functional of the fluid composition field with a Navier-Stokes expression for the hydrodynamic flow field, compounded with cross-terms coupling the dynamics of the fields [8,34]. Although this model is beyond analytic solution, it is generally accepted that the domains formed in the spinodal decomposition of a symmetric binary fluid follow a scaling law, i.e. the patterns at early and late times are statistically similar in structure and differ by a scale factor only. The evolution of the single relevant length scale is believed to follow a power law, $R(t) \propto t^{\alpha}$, where the growth exponent $\alpha$ is determined by equating the dominant terms in model $\mathrm{H}$. Since the importance of the various terms in this model depends on $R$ and $\dot{R}$, it follows that the growth exponent passes through three consecutive regimes: an initial diffusion-dominated $\alpha=1 / 3$ period [57], a regime of interfacial-driven viscous growth where $\alpha=1$ [79] and a late stage of interfacial-driven inertial growth with $\alpha=2 / 3$ [27,44]. The linear regime is ubiquitous in the experiments [30,36,99], diffusive growth has been observed for shallow quenches near the critical point $[12,100]$, while the existence of the late stage has only been confirmed recently in Lattice Boltzmann (LB) simulations [45]. The two earlier stages have also been studied in computer simulations, by using dedicated Navier-Stokes solvers like LB and lattice gas automata [2,46, 69], and by off-lattice particle-based methods including molecular dynamics (MD) and dissipative particle dynamics (DPD) [15,41, 42, 53, 59, 89].

Other spinodal decomposition processes are less well understood than the idealised situation outlined above, although the basic principles guiding the ongoing investigations remain the same. Dynamical asymmetry of the two mixed fluids, meaning that their viscosities 
differ significantly or that one component shows viscoelastic behaviour, explains the complex phase separation processes and the 'phase inversion' phenomenon observed in polymersolvent mixtures and colloidal suspensions $[5,86,88]$. In these systems the 'slow' component can not keep up with the growth rate imposed by the 'fast' component, a network enriched in the slow component forms and subsequently succumbs to internal stresses to arrive again at a regular phase separated final configuration. An externally imposed shear flow both accelerates and hinders the phase separation process, by the continuous transport, elongation and resulting ruptures of the domains $[11,67]$. In combination with the interfacial driving force, these give rise to anisotropic growth of the domains, with two linear growth processes in the flow-vorticity plane and a possibly supralinear growth, $\alpha>1$, in the shear gradient direction $[11,14,54]$. The domains can not grow indefinitely in the shear-gradient direction, but will attain a rate-dependent nonequilibrium steady state; it is not clear whether the growth in the two perpendicular directions also saturates $[11,84]$. Shear may also be applied to induce phase separation in dynamically asymmetric mixtures $[20,50]$. Deviations from the ideal case also occur in the presence of walls and other fixed obstacles, which limit the attainable domain size, interfere with the build-up of a hydrodynamic flow field, and may have a preference for wetting by either constituent of the mixture [70,87,90]. Another interesting spinodal decomposition process has been reported for rod-like colloids, where the nematic (dis)ordering enslaves the spatial ordering into dense and less-dense phases $[19,56]$.

Experimental investigations of spinodal decomposition are traditionally aimed at studying one of the above decomposition processes, by specifically designing the equipment and conditions such as to eliminate or minimize perturbing side effects. Occasionally two factors are taken into account in one experiment, e.g. dynamically asymmetric mixtures under shear $[38,50]$, to study the synergy or competition between the two mechanisms involved in these phase separation processes. But with the rapid advances in 'microfluidics' - the field studying flow processes in individual liquid droplets and extremely narrow channels $[64,85]-$ there are many conceivable experiments in which several of the aforementioned mechanisms are simultaneously involved in the spinodal decomposition process. In a microfluidic rheology experiment on a polymer-solvent mixture using a miniaturized Taylor-Couette cell, for instance, the kinetics of the phase separation process is determined by dynamic asymmetry, non-uniform shear, confinement and wetting, in addition to the unequal attractions between 
the molecules. Computer simulations provide a preview on the intricate phase separation processes that arise under these complex conditions.

In this work we study phase separation of polymer-solvent and rod-solvent mixtures in a miniature Taylor-Couette cell, extending our previous simulations on binary fluids in the same geometry [90]. Details on the employed models and simulation techniques are summarized in Section 4.2. The simulation results, both for stationary walls and for a rotating inner wall, are presented and discussed in Section 4.3 for a wide range of systems. By necessity, our results and the deliberations will mostly be of a qualitative nature. The main conclusions distilled from the simulations are summarized in Section 4.4.

\subsection{Simulation details}

In the current simulation study, the non-bonded interaction between two like particles $i$ and $j$ with relative positions $\mathbf{r}_{i}-\mathbf{r}_{j}=r_{i j} \hat{\mathbf{r}}_{i j}$, with the hat indicating a unit vector, is modelled by the usual Lennard-Jones potential,

$$
U_{\mathrm{LJ}}\left(r_{i j}\right)=4 \varepsilon\left[\left(\frac{\sigma}{r_{i j}}\right)^{12}-\left(\frac{\sigma}{r_{i j}}\right)^{6}\right],
$$

where $\varepsilon$ and $\sigma$ are the strength and range, respectively. Discontinuities in the potential and force close to the cut-off distance, $r_{c}=2.5 \sigma$, are removed by a smooth truncation of the potential. A purely repulsive Weeks-Chandler-Andersen (WCA) potential, $U_{\mathrm{WCA}}\left(r_{i j}\right)=$ $U_{\mathrm{LJ}}\left(r_{i j}\right)+\varepsilon$ for $r_{i j} \leq 2^{1 / 6} \sigma$ and $U_{\mathrm{WCA}}\left(r_{i j}\right)=0$ for $r_{i j}>2^{1 / 6} \sigma$, acts between pairs of unlike particles. A polymer $P_{n}$ is created by the expedient of stringing $n$ solvent particles $S$ together via a finitely extensible nonlinear elastic (FENE) potential,

$$
U_{b}\left(r_{i, i+1}\right)=-\frac{1}{2} k_{b} r_{\infty}^{2} \ln \left[1-\left(\frac{r_{i, i+1}}{r_{\infty}}\right)^{2}\right] .
$$

With the bond constant set to $k_{b}=30 \varepsilon / \sigma^{2}$ and the maximum length of the bond limited to $r_{\infty}=1.5 \sigma$, the resulting polymers are very flexible, as shown by their persistence length of nearly $1 \sigma$. A more rigid chain, $R_{n}$, resembling a polymeric liquid crystal, is made by introducing a stiffening potential between next-nearest neighbours in a chain,

$$
U_{s}\left(r_{i, i+2}\right)=\frac{1}{2} k_{s}\left(r_{i, i+2}-r_{s}\right)^{2} .
$$


For $k_{s}=10 \varepsilon / \sigma^{2}$ and $r_{s}=4 \sigma$, the persistence length of the chain increases to $17 \sigma$, which amounts to about three times the chain length. The classical mechanics equations of motion are integrated numerically using the Verlet leap-frog algorithm with a time step $\Delta t=0.002 \tau$, where the unit of time is defined by $\tau=\sqrt{m \sigma^{2} / \varepsilon}$. All particles have the same mass $m$.

A thermostat is employed to maintain a steady temperature $T=1 \varepsilon / k_{B}$, where $k_{B}$ is the Boltzmann constant. Without a thermostat the system would gradually heat up due to the energy released during phase separation and the work exerted on the system by the rotating wall. Since hydrodynamic interactions are essential in the proper simulation of the later stages of spinodal decomposition, as well as in the simulation of flow phenomena, care was taken to select a hydrodynamics conserving thermostat. Such a thermostat was introduced as part of the Dissipative Particle Dynamics (DPD) method [16, 29, 35], by implementing friction and random forces as momentum conserving interactions between pairs of particles. In short, these forces are expressed as

$$
\mathbf{F}_{i j}^{d+r}=-\frac{\kappa^{2}}{2 k_{B} T}\left(1-\frac{r_{i j}}{r_{c}}\right)^{2}\left(\hat{\mathbf{r}}_{i j} \cdot \Delta \mathbf{v}_{i j}\right) \hat{\mathbf{r}}_{i j}+\frac{\kappa}{\sqrt{\Delta t}}\left(1-\frac{r_{i j}}{r_{c}}\right) \zeta_{i j} \hat{\mathbf{r}}_{i j},
$$

where $\kappa=3.0 \varepsilon \tau^{1 / 2} \sigma^{-1}$ determines the strength of the thermostat, $r_{c}=2.5 \sigma$ is the cutoff distance and $\Delta \mathbf{v}_{i j}$ is the velocity difference between two particles. The $\zeta_{i j}(t)$ denotes a random number with zero mean and unit variance, sampled independently and without correlation for every pair at every time step. A fluctuation-dissipation theorem, relating the dissipative first term to the stochastic second term via the temperature $T$, is usurped in the above expression.

A Taylor-Couette geometry is created by confining the fluid particles between two coaxial cylindrical walls. In the simulations presented here, the outer wall is always stationary while the inner wall is either stationary or rotating at a constant angular velocity $\omega_{i}$. For any particle $i$ crossing a wall during the integration time step, the velocity $\Delta \mathbf{v}_{i}$ of the particle relative to that wall is reversed at the moment of collision, $\Delta \mathbf{v}_{i} \mapsto-\Delta \mathbf{v}_{i}$, before executing the remainder of the time step. This bounce-back rule is introduced with the aims of restricting the particles to the Couette cell and creating stick boundary conditions at both walls, but in the simulations, especially those at high angular velocities or with viscous fluids, some slippage remains. It is therefore augmented with a scheme to re-sample the velocities of all particles within $1 \sigma$ of a wall from a Maxwell-Boltzmann distribution, offset by the rotational velocity of the 
wall; by applying this procedure every time step the desired non-slip boundary conditions are recovered.

In order to reduce the computational demands of the simulation, we do not simulate the entire Taylor-Couette cell but a section amounting to one-sixth of the cell, i.e. a segment covering an angle $\Theta_{t}=\pi / 3$, with periodic boundary corrections applied along the azimuthal direction. These corrections are readily implemented in a Cartesian-coordinate based simulation code [90], the only minor complication being that any calculation involving a boundary crossing term now involves a rotation over $\pm \Theta_{t}$ around the central axis of the Couette cell. Regular periodic boundary conditions apply along the axial direction.

The inner wall $R_{i}$ is set at either $25 \sigma$ or $40 \sigma$, the outer wall $R_{o}$ takes on a range of values from $40 \sigma$ to $75 \sigma$. The systems with the smaller inner radius contain $N=10500$ particles, simulations with 15-bead chains are executed with 30000 particles, while all other systems contain 21000 particles. Both solvent and polymer particles are always present in equal numbers. The particle density was fixed at $\rho=0.7 \sigma^{-3}$, resulting in box heights $h=2 N /\left[\rho \Theta_{t}\left(R_{o}^{2}-R_{i}^{2}\right)\right]$ varying from $15.3 \sigma$ to $31.2 \sigma$.

In rheological experiments employing a Couette cell, the obvious quantities of interest are the total torque exerted by the rotating inner wall and the amount of work required to maintain its steady angular velocity. The relevant shear stress at the inner wall is calculated in our simulations from

$$
\tau_{r \theta}\left(R_{i}\right)=-\frac{1}{\Theta_{t} h R_{i}^{2}}\left\langle\sum_{i} m_{i} r_{i} v_{i \theta} v_{i r}+\sum_{i} \sum_{\substack{j \\ j>i}} \bar{r}_{i j}\left(r_{i}-r_{j}\right)\left[\mathbf{F}_{i j}^{c+d} \cdot \hat{\mathbf{e}}_{\theta}\left(\overline{\mathbf{r}}_{i j}\right)\right]\right\rangle
$$

where the pointed brackets denote a temporal average, $v_{i r}=\mathbf{v}_{i} \cdot \hat{\mathbf{e}}_{r}\left(\mathbf{r}_{i}\right)$ the radial velocity and $v_{i \theta}=\mathbf{v}_{i} \cdot \hat{\mathbf{e}}_{\theta}\left(\mathbf{r}_{i}\right)$ the azimuthal velocity of particle $i$, and $\overline{\mathbf{r}}_{i j}=\left(\mathbf{r}_{i}+\mathbf{r}_{j}\right) / 2$. The superscript to the pair force emphasizes that both conservative and dissipative forces are needed in the stress calculation. The power supplied by the inner wall in the steady state is given by $P=$ $\omega_{i} \Theta_{t} h R_{i}^{2} \tau_{r \theta}\left(R_{i}\right)$ and equals the rate of energy dissipation by viscous effects in the sheared fluid. A derivation of these expressions are presented in the appendix. 


\subsection{Results}

In this section an extensive survey is given of the simulated phase separation processes in a Couette geometry. To structure the discussion, the fluid properties will be analysed first. Next comes phase separation in a quiescent mixture, studying the final stable states and their rates of formation. We end with phase separation under shear, again describing both the stationary states and the evolutions bringing the mixed systems to these states.

\subsubsection{Characterization of the fluids}

The most important fluid parameters in the simulations of phase separation in a Couette flow are the interfacial tensions and the viscosities; these parameters are easiest calculated from simulations of cubic boxes. A system with regular 3D periodic boundary conditions then phase separates into two parallel flat layers, and one readily shows, from the free energy change under a volume-conserving area variation, that the interfacial tension $\gamma$ follows from

$$
\gamma=\left(\frac{\partial F}{\partial L_{\|}^{2}}\right)_{N V T}=\frac{1}{2}\left(p_{\|}-p_{\perp}\right) L_{\perp} .
$$

Here $p$ denotes the pressure, with the subscripts $\perp$ and $\|$ indicating the directions perpendicular and parallel to the interface, respectively, and $L$ is the edge length of the box. The factor of a half on the right hand side arises because a 3D periodic box contains two interfaces. Fluid-wall interfacial tensions are calculated in a similar way, using a two-directional periodic box with hard walls in the third direction. The surface tensions of the various interfaces are summarized in Table 4.1. Visual inspection of the fluid-fluid simulations, using the Visual Molecular Dynamics (VMD) program [37], reveals that the polymer and rod phases are denser than their coexisting solvent phases by some $10 \%$. A similar observation is made in the Couette geometry. We attribute this increased density to the bonds between the particles, which reduce the nearest neighbour distances between particles in the same polymer. In order to correct for the possible effects of these density differences, the fluid-wall interfacial tensions listed in Table 4.1 have been calculated at the specific densities found in the fluid-solvent simulations.

The viscosities of the various fluids are readily obtained from shear simulations in conventional rectangular boxes as the proportionality constant relating the shear stress $\tau_{x z}$ to 
Table 4.1: Surface tensions $\gamma_{S X}$ of the solvent-fluid interface and $\gamma_{W X}$ of the wall-fluid interface, and the fluid viscosities $\eta_{X}$, as obtained from simulations of cubic systems. Columns four and five contain the densities $\rho_{S}$ of the solvent and $\rho_{X}$ of the fluid in the solvent-fluid systems. All wall-fluid interfacial tensions are calculated at the denoted fluid density $\rho_{X}$. The solvent-wall interfacial tensions for the two relevant solvent densities are reported in the first two rows.

\begin{tabular}{cccccc}
$X$ & $\gamma_{S X} / \varepsilon \sigma^{-2}$ & $\gamma_{W X} / \varepsilon \sigma^{-2}$ & $\rho_{S} / \sigma^{-3}$ & $\rho_{X} / \sigma^{-3}$ & $\eta_{X} / \varepsilon \tau \sigma^{-3}$ \\
\hline$S$ & $1.23^{1}$ & 0.53 & 0.70 & 0.70 & 8.4 \\
$S$ & - & 0.41 & - & 0.64 & 6.4 \\
$P_{7}$ & 1.40 & 0.64 & 0.64 & 0.78 & $36^{2}$ \\
$P_{15}$ & 1.38 & 0.69 & 0.64 & 0.78 & \\
$R_{7}$ & 1.37 & 0.39 & 0.64 & 0.78 & $\sim 55^{2}$ \\
$R_{15}$ & 1.28 & 0.51 & 0.64 & 0.78 &
\end{tabular}

the shear rate $\dot{\gamma}$. Linear shear flows $\mathbf{v}(\mathbf{r})=\dot{\gamma}_{z} \hat{\mathbf{e}}_{x}$ are introduced by applying Lees-Edwards moving boundary conditions, as well as by placing a two-directional periodic fluid between one stationary and one linearly moving wall. In the latter case, the stress tensor is calculated excluding the regions within $2 \sigma$ of the walls to avoid complications from the stick-wall procedure. Both sets of simulations yield similar results, indicating that the stick conditions at the walls are working satisfactorily. The viscosities are listed in Table 4.1. Both the polymers and the rods exhibit shear thinning, with $\eta_{P_{7}} \approx 36 \varepsilon \tau \sigma^{-3} /\left[1+(16 \tau \dot{\gamma})^{-0.94}\right]$ and $\eta_{R_{7}} \approx 61 \varepsilon \tau \sigma^{-3} /\left[1+(48 \tau \dot{\gamma})^{-0.74}\right]$ for shear rates in the range $(1-50) \times 10^{-3} \tau^{-1}$. Clearly, this further complicates the nature of the phase separation process under shear, as it may have consequences for both the growth rate and the resulting steady state.

In their homogeneous phase, the stiff $R_{7}$ and $R_{15}$ chains show a hallmark of liquidcrystals: the rods spontaneously align to establish a nematic ordering. The order parameter $S_{2}$, defined as the largest eigenvalue of the matrix

$$
\mathbf{S}=\frac{3}{2}\left(\left\langle\hat{\mathbf{u}}_{j} \hat{\mathbf{u}}_{j}\right\rangle-\frac{1}{3} \mathbf{I}\right)
$$



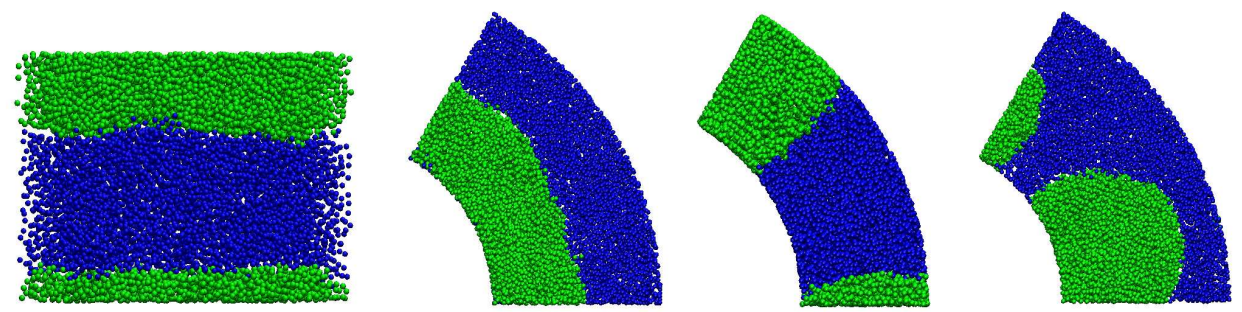

Figure 4.1: Snapshots of four typical phase-separated final configurations, showing (from left to right) a $z$-stack, an $r$-stack, a $\theta$-stack and a droplet attached to the inner wall. The $z$ stack is shown in a side view, with the axis of the Couette cell running vertically, while the other configurations are top views looking down along the rotation axis of the cell. Because of the Couette cell's rotational symmetry, we simulate a segment amounting to one-sixth of the entire cell by using periodic boundary conditions in the axial and azimuthal directions. Polymer particles are coloured green, solvent particles are blue; note that the particles are not drawn to scale and that the dimensions of the cell vary in the four systems.

where $\hat{\mathbf{u}}_{j}$ denotes the bond vectors in the rods and $\mathbf{I}$ is the unit matrix, rises to about 0.6 on a scale from 0 for purely random to 1 for completely aligned. No such ordering was observed in the homogeneous solvent-rod systems with Lennard-Jones interactions between all particles, because the isotropic phase is either stable or a long-lived metastable state.

\subsubsection{Steady state at zero shear}

Based on the geometry of the Couette cell, the orientations of the fluid - fluid interfaces in a fully phase separated state are likely to correspond to the basis vectors in cylindrical coordinates. Stable states with interfacial normals along the radial direction $\hat{\mathbf{e}}_{r}$ will henceforth be denoted as $r$-stacks, states with normals parallel to the axial axis $\hat{\mathbf{e}}_{z}$ are called $z$-stacks and normals in the azimuthal direction $\hat{\mathbf{e}}_{\theta}$ are $\theta$-stacks. Snapshots illustrating these stacks are shown in Fig. 4.1. The calculated surface tensions then permit a straightforward approximate determination of the thermodynamically stable states, by finding the state with the lowest free energy among the anticipated optimum configurations. Here it is implied that interfacial 
contributions dominate the free energy differences between the various stackings of a given mixture, while variations in the bulk contributions are deemed negligible.

A simple surveying of the various surface contributions, where one may conveniently assume idealized flat or uniformly-curved interfaces, then yields the free energies for every mixture and stacking. Corrections for the density differences between the coexisting fluids are readily implemented. The example depicted in Fig. 4.2 concerns one of the simulated systems, namely the $P_{7}-S$ system containing 21000 particle and a cell with an inner wall radius $R_{i}=40 \sigma$. On the basis of this plot, we expect the simulations of this system to yield

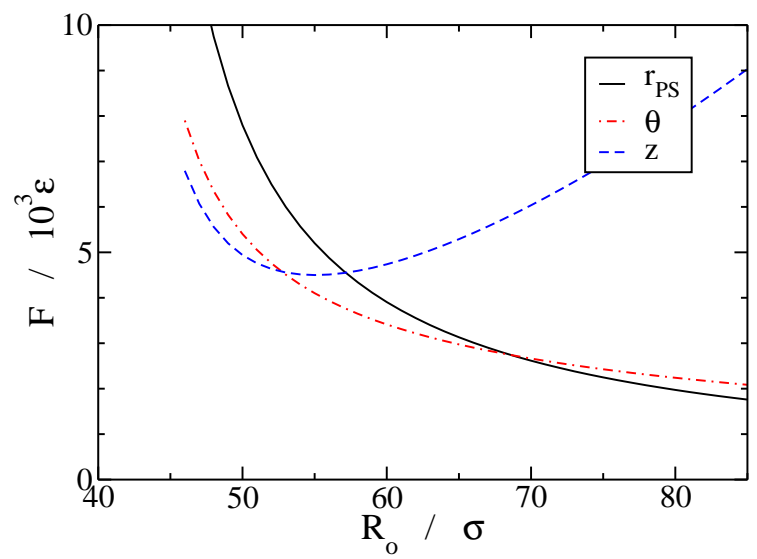

Figure 4.2: The total interfacial energies of three different stackings in a phase separated polymer-solvent system, $P_{7}-S$, as a function of the radius $R_{o}$ of the outer wall. All parameters entering the calculation of these theoretical curves are based on the simulated system with an inner wall radius $R_{i}=40 \sigma$.

$z$-stacks for outer wall radii up to about $52 \sigma, r$-stacks beyond approximately $69 \sigma$, and $\theta$ stacks in the intermediate region. A comparison with the simulation results is described in the next subsection.

Due to the different fluid-wall interfacial tensions, see Table 4.1, most fluid-fluid interfaces will impinge on the wall under a non-perpendicular contact angle, following Young's law [74], and the fluid-fluid interfaces will consequently be non-uniformly curved. Theoretical expressions for the interfacial shape in a Couette cell have been derived in [90], 


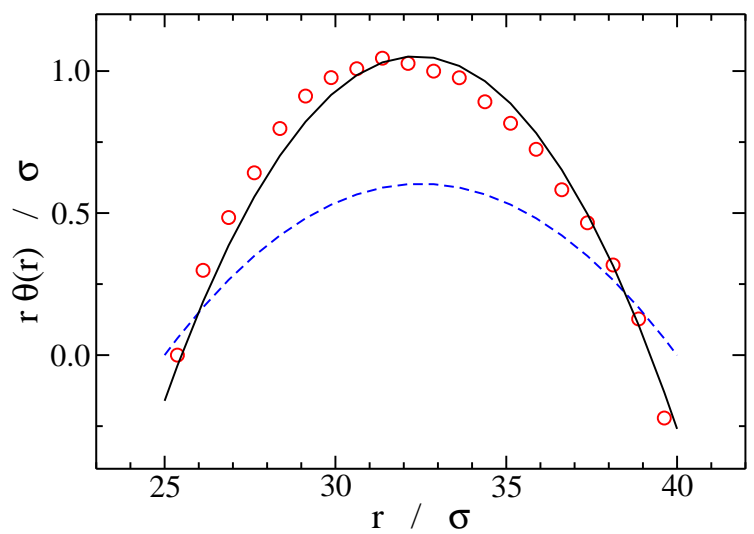

Figure 4.3: The profile of the polymer - solvent interface obtained from simulations (circles) of a $P_{7}-S$ system $\left(R_{i}=25 \sigma, 21000\right.$ particles). The theoretical curve (dashed line) of Eq. (4.8) is calculated with $\Gamma=0.16$, based on the interfacial tensions in Table 4.1, while the optimum fit (solid line) is achieved at $\Gamma=0.31$.

where it was found that the surface tensions consistently enter the expressions in the form $\Gamma=\left(\gamma_{A W}-\gamma_{B W}\right) / \gamma_{A B}$, with $A, B$ and $W$ denoting the two fluids and the wall, respectively. For a $\theta$-stack, for instance, the interface is described by

$$
\theta(r)=\theta\left(R_{i}\right)+\int_{R_{i}}^{r} \frac{C+\lambda x^{2}}{\sqrt{x^{4}-x^{2}\left(C+\lambda x^{2}\right)^{2}}} d x,
$$

where $\lambda=\Gamma /\left(R_{i}-R_{o}\right)$ and $C=\Gamma R_{i} R_{o} /\left(R_{o}-R_{i}\right)$. Note that density differences between the two fluids affects the location of the interface, i.e. $\theta\left(R_{i}\right)$, but have no influence on the shape of the interface. The predicted interface is depicted in Fig. 4.3, for $\Gamma=0.16$, along with the actual interface of a polymer-solvent system. We have calculated this profile by using a 2-dimensional grid to establish the average densities $\rho_{P}(r, \theta)$ and $\rho_{S}(r, \theta)$ of polymer and solvent particles, respectively. The order parameter field for a given grid value of $r$,

$$
\phi_{r}(\theta)=\frac{\rho_{P}(r, \theta)-\rho_{S}(r, \theta)}{\rho_{P}(r, \theta)+\rho_{S}(r, \theta)}
$$

is then numerically fitted with a sum of two theoretical interfacial profiles,

$$
\phi_{r}^{\text {theo }}(\theta)=\alpha_{r} \tanh \left[\beta_{r} r\left(\theta-\theta_{1}\right)\right]-\alpha_{r} \tanh \left[\beta_{r} r\left(\theta_{2}-\theta\right)\right],
$$


to yield the profiles $\theta_{1}(r)$ and $\theta_{2}(r)$ of the two polymer - solvent interfaces. Figure 4.3 shows the moderate agreement of their average with the predicted interfacial shape, suggesting that other factors, such as the connectivity of the beads in the polymers, might play subtle roles as well. The deviations from a flat interface are small, however, and of little consequence in the area-based free energy calculations of Fig. 4.2. As concerns the remaining two fit parameters, $\alpha_{r}$ approximately equals unity while $\beta_{r}^{-1} \approx 2 \sigma$ measures the combined effects of the interfacial width and temporal variations in the location of the interface.

The simulations of spinodal decomposition of binary fluid mixtures in a Couette geometry are first performed for stationary walls, i.e. without the added complications of a non-uniform shear flow. For each mixture, a start configuration is created by randomly placing the fluid particles in the simulation box, subject to the conditions imposed by bonds between connected particles; trial positions resulting in significant pair overlaps are rejected. The systems are then equilibrated by running extensive simulations at the desired equilibrium temperature, using regular Lennard-Jones interactions between all particles to suppress phase separation of the two fluid components. A subsequent deep quench to an unstable state is created by instantaneously introducing purely repulsive WCA interactions between unlike particles, thus initiating the spinodal decomposition of a homogeneous mixture. Visual inspection of the ensuing simulation dynamics clearly shows the formation of a fine network of regions rich in either of the fluids. The network gradually coarsens, as the domains grow in size and the connections between the domains break one by one, until the system eventually arrives in a steady state consisting of two fully separated regions. The final states of some thirty systems, varying in fluid-fluid compositions and Couette geometry, are collected in Table 4.2, with snapshots of typical end configurations shown in Fig. 4.1. In most cases the orientations of the fluid - fluid interfaces corresponds to one of the basis vectors in cylindrical coordinates, i.e. $\hat{\mathbf{e}}_{r}$ for $r$-stacks, $\hat{\mathbf{e}}_{z}$ for $z$-stacks or $\hat{\mathbf{e}}_{\theta}$ for $\theta$-stacks. A significant fraction of the simulations has ended up in less regular states: some adopted an other stack conformation, several runs produced a droplet $(d)$ attached to a wall, while still other runs appear stuck in an intermediate network state $(n)$ attached to both walls at multiple positions. Table 4.2 shows a moderately satisfactory agreement between the steady states in the simulations and the predictions based on the interfacial free energies. Several factors may contribute to the deviations, which are more abundant here than for previous simulations of a fluid-fluid mix- 
Table 4.2: Phase separated steady states in a stationary nanoscopic Taylor-Couette cell, for various drum geometries (collected in rows, with $R_{i}$ and $R_{o}$ the inner and outer radii of the cell) and various binary fluid mixtures (sorted in columns, with $S$ for solvent, $P$ for polymer and $R$ for rod). Each state is coded according to the direction of its fluid-fluid interfacial normals, which predominantly point in the radial $(r)$, azimuthal $(\theta)$ or axial $(z)$ direction, with snapshots illustrating these stacks given in Fig. 4.1. Subscripts to r-stacks indicate the sequential order of layers traversed when going from the inner to the outer wall of the Couette cell. Droplets are marked d, with the superscript distinguishing between droplets attached to the inner ( $i)$ or outer (o) wall, and the subscript giving the composition of the droplet. Long-lived bicontinuous networks spanning the entire cell are registered as $n$. Three systems in the top-left corner of the table were run four times each, with the results of these independent simulations collected in a comma-separated list, to give an impression of the repeatability of the final state. Bracketed entries showing the stable state according to a free energy calculation, as discussed in Section 4.3.2, are included if the predicted state differs from the observed final state.

\begin{tabular}{cc|cccccc}
$R_{i} / \sigma$ & $R_{o} / \sigma$ & $P_{7}-S$ & $R_{7}-S$ & $P_{7}-R_{7}$ & $R_{7}-R_{7}$ & $P_{15}-S$ & $R_{15}-S$ \\
\hline 25 & 40 & $z, z, z, z(\theta)$ & $z, z, z, \theta(\theta)$ & & $z(\theta)$ & & \\
25 & 50 & $d_{P}^{i}, d_{P}^{i}, n, r_{S P S}\left(r_{P S}\right)$ & $r_{R S}$ & & & & \\
40 & 60 & $z(\theta)$ & $\theta$ & $\theta$ & & & \\
40 & 65 & $\theta$ & $\theta$ & $d_{P}^{o}(\theta)$ & $\theta$ & $r_{P S}(\theta)$ & $n(\theta)$ \\
40 & 70 & $\theta\left(r_{P S}\right)$ & $\theta\left(r_{R S}\right)$ & $d_{R}^{o}(\theta)$ & & $r_{P S}$ & $n\left(r_{R S}\right)$ \\
40 & 75 & & & & & $d_{P}^{o}\left(r_{P S}\right)$ & $n\left(r_{R S}\right)$
\end{tabular}


ture in a micro-Couette cell [90]. The assumption that the surface tensions dominate the free energy, ignoring all other contributions, is approximate by nature, and bending contributions to the interfacial free energy are neglected. The surface tensions have been calculated for flat interfaces, and are perhaps less suited for the current curved interfaces, as examplified by the interfacial profile in Fig. 4.3. A recalculation, using the fitted $\Gamma$ in combination with $\gamma_{S W}$ and $\gamma_{S P}$, does not significantly alter the predictions. It is well conceivable that the walls steer the initial phase separation in a certain direction, thereby setting the system onto a path towards a sub-optimal final state. And lastly, since the emerging decomposition pattern varies with the quenched start configuration, the final state is to a certain degree inherently unpredictable: this indeterminism usually manifests itself in the locations of the domains, but perhaps the chaotic origin suffices in the current conditions to co-determine the final state. To assess this reproducibility of the separation process, we have simulated three systems several times using independently generated homogeneous starting configurations. The results, see Table 4.2, indicate that certain systems consistently arrive at the same final state, while other systems yield multiple steady states. In conclusion, the thermodynamically optimum state yields a moderately accurate prediction, but it can not be completely relied on for a number of reasons.

In the case of $r$-stacks, both the flexible and rigid chains systematically cover the inner wall with the solvent occupying the outer region of the cell. This configuration is stabilised by a minimisation of the total fluid-wall interfacial energy, which drives the chains, with their higher surface tensions, $\gamma_{W P}>\gamma_{W S}$, to the wall with the smaller area. Since the densities of the chain fluids are higher than the density of the solvent, the configuration with the chains on the inside also has the smallest chain-solvent interfacial area, which also contributes to the stability of this configuration.

Besides both the three expected types of stacks, Table 4.2 also includes a number of additional long-lived conformations. Several simulations ended with a droplet of the dense polymer or rod phase attached to either the inner or the outer wall of the Couette cell. These droplets are always oriented parallel to the drum axis and span the entire height of the simulation box, suggesting that the periodic boundary condition along $z$ contributes to their stability [17] and hence that droplets are less likely in experiments. Droplets surrounded by a solvent phase prove very stable in our simulations, indicating a high activation barrier for the 
eventual transition to a regular stacked configuration, which most likely will be a $\theta$-stack. The droplets observed in the polymer-rod mixtures are larger than those exposed to solvent because here both separated phases have approximately equal densities. Figure 4.1 illustrates how these droplets resemble meta-stable states, which can evolve either into a $\theta$-stack, by stretching in the radial direction towards the opposite wall, or into an $r$-stack, by bridging the gap separating the droplet from its periodic image. In the simulations with the long $R_{15}$ rods, the mixture forms an irregular network of the rod phase within about $4 \times 10^{3} \tau$, and remains unchanged till the termination of the run some $6 \times 10^{3} \tau$ later. This suggests that the rods fluid relaxes relatively slowly, compared to the polymer melts, which will be the topic of the next subsection.

\subsubsection{Decomposition dynamics at zero shear}

In order to get a first impression of the rates of domain formation, we have simulated spinodal decomposition in a periodic cubic box of sides $L=48 \sigma$, using the procedures outline above. The employed binary mixtures are $S-S, P_{7}-S$ and $R_{7}-S$, again with equal numbers of each type of particle. Time-dependent average domain sizes $R(t)$ have been determined by means of structure factor calculations, using the procedure outlined in [89]. Figure 4.4 shows the growth curves of the three mixtures, as obtained by averaging five independent simulations for each mixture. It is evident that the binary liquid mixture separates much faster than the polymer and liquid crystal mixtures, and that the curves are all fairly similar in shape. Since the growth curves $R(t)$ of symmetric binary mixtures are believed to coalesce to a master curve after appropriate adjustments of the time and length scales, we have followed a similar procedure to maximise the agreement between the current three curves. From the optimizing scaling factors it follows that the $P_{7}-S$ mixture separates about 1.8 times as fast as the $R_{7}-S$ mixture. This difference is most likely caused by the rigidity of the rods, which may hinder their motions, since both mixtures have comparable interfacial tensions. In addition, the stiff polymers show signs of nematic ordering in the dense polymer phase. Based on the three curves, we estimate the transition from diffusive $R \propto t^{1 / 3}$ to interfacial-driven viscous growth $R \propto t$, to happen around a time $t_{d v}$ of about $10 \tau$ for the binary solvent mixture, at $\sim 40 \tau$ for the polymer-solvent mixture, and at around $70 \tau$ for the rod-solvent mixture.

In the absence of a straightforward quantization method for domain sizes in a Couette 


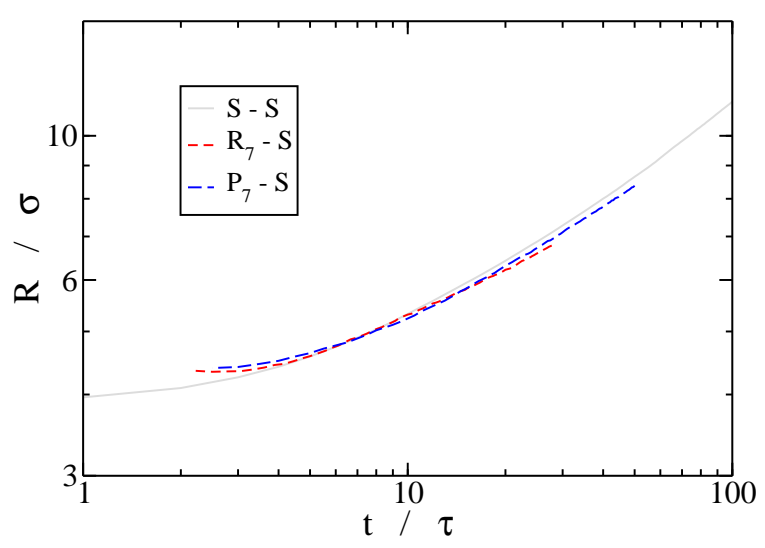

Figure 4.4: Average domain size R as a function of time t for spinodal decomposition in three fluid mixtures. The $P_{7}-S$ curve is scaled along the horizontal axis by a factor of 4 and along the vertical axis by a factor of 2, to make the curve coalesce with the $S-S$ curve. Similar scaling-factors of 7 and 2 have been applied to the $R_{7}-S$ curve.

geometry, we have to resort to visual inspection of the emerging patterns to get an impression of the relative growth rates of different binary mixtures. A series of snapshots of phase separation in $P_{7}-S$ and $R_{7}-S$ are collected in Fig. 4.5. Because of the anticipated scaling factor of 1.8, the snapshots of the liquid crystal system are taken at correspondingly later times than those of the polymer system. By comparing the two series, it appears that the growth rates indeed differ by a factor of about 1.8 during the initial stages of phase separation. For later times, however, the rod mixture is clearly lagging behind even more: the connected network of rod domains survives much longer than the network of polymer domains. A similar slowing down arises for the longer $R_{15}$ rods, which are still in a network phase by the time the corresponding $P_{15}$ polymers have already fully phase separated into their steady state.

\subsubsection{Steady state under shear}

The binary fluid mixtures and cell geometries of Section 4.3.2 have also been simulated with a rotating inner wall. The final steady states observed in these simulations, which again 

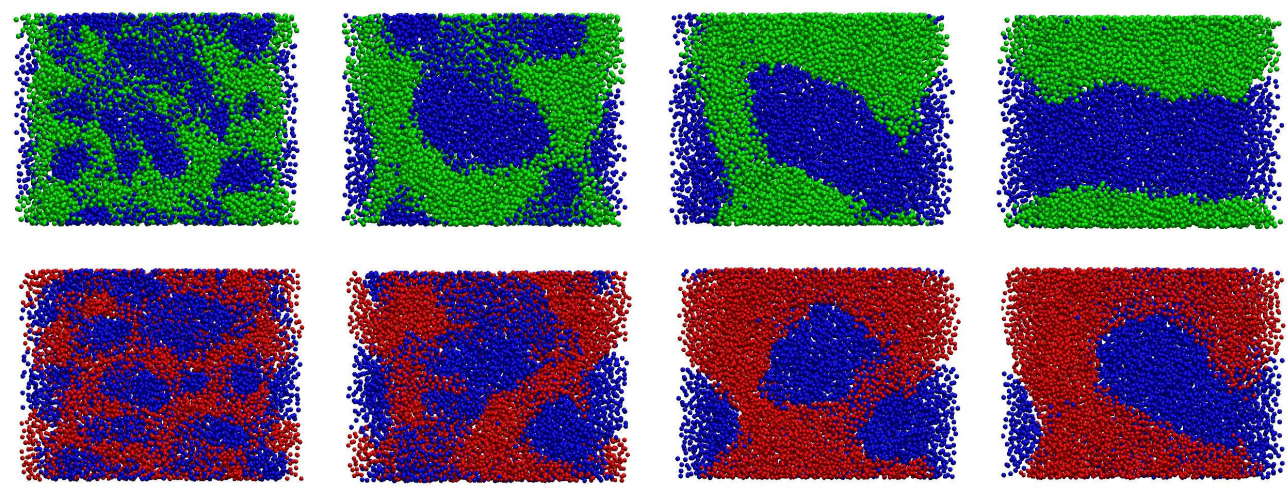

Figure 4.5: Side views of polymer-solvent (top, at $t=100 \tau, 500 \tau, 1500 \tau$ and $2500 \tau$ ) and rod-solvent (bottom, at $t=175 \tau, 875 \tau, 2625 \tau$ and $4375 \tau$ ) mixtures showing various stages during their respective phase separations in Couette cells with $R_{i}=25 \sigma$ and $R_{o}=40 \sigma$. The difference in the time scaling by a factor of $1 \frac{3}{4}$ compensates for the anticipated slower segregation of the rods in a cubic periodic box, see Fig. 4.4.

started from a homogeneously mixed initial state, are summarised in Table 4.3 for inner-wall angular velocities $\omega_{i}$ of $10^{-3} \mathrm{rad} / \tau$ and $10^{-2} \mathrm{rad} / \tau$. Several trends stand out in Table 4.3 and these will be discussed in this subsection in some detail. We start by noting that a rough indication of the corresponding shear rates is given by $\dot{\gamma} \approx \omega_{i} R_{i} /\left(R_{o}-R_{i}\right)$ and ranges from $10^{-3} \tau^{-1}$ to $2 \times 10^{-2} \tau^{-1}$, while the actual shear rate is of course non-uniform in a Couette cell. The shear rates are therefore relatively small compared to the transition times between consecutive growth regimes, with $\dot{\gamma} t_{d v}<1$ for most systems, implying that the spinodal decomposition reaches a growth regime driven by interfacial-tension. Consequently, we find large domains whose dimensions are not limited by the shear, even at the highest attempted drum velocity of $\omega_{i}=5 \times 10^{-2} \mathrm{rad} / \tau$. From Table 4.3 it is clear that $\theta$-stacks, which are abundant in the absence of shear, are not being formed under shear. An existing $\theta$-stack connecting the two opposing walls will, under the influence of a rotating inner cylinder, be wrapped around the inner drum and simultaneously be stretched thin, and subsequently be torn apart under the mounting elongational strain. It is not surprising, therefore, that $\theta$-stacks 
Table 4.3: Steady states of phase separated binary mixtures in a nanoscopic Taylor-Couette cell for inner walls rotating at an angular velocity $\omega_{i}=0.001 \mathrm{rad} / \tau$ (top half of the table) or $\omega_{i}=0.01 \mathrm{rad} / \tau$ (bottom half of the table) and a stationary outer wall. See Table 4.2 for an explanation of the notation. Double and triple banded r-stacks are distinguished by their number of subscripts. The steady states marked $r_{X S}+d_{X}^{o}$ comprise a layer of polymers or rods $(X)$ covering the inner wall and a droplet of the same composition attached to the outer wall.

\begin{tabular}{cccccccc}
$R_{i} / \sigma$ & $R_{o} / \sigma$ & $S-P_{7}$ & $S-R_{7}$ & $P_{7}-R_{7}$ & $R_{7}-R_{7}$ & $S-P_{15}$ & $S-R_{15}$ \\
\hline 25 & 40 & $z$ & $z$ & & $z$ & & \\
25 & 50 & $d_{P}^{o}$ & $r_{R S}+d_{R}^{o}$ & & & & \\
40 & 60 & $r_{P S}$ & $r_{R S}$ & $d_{R}^{o}$ & & & \\
40 & 65 & $r_{P S}$ & $d_{R}^{o}$ & $r_{P R P}$ & $z$ & $r_{P S}$ & $z$ \\
40 & 70 & $d_{P}^{o}$ & $r_{S R S}$ & $r_{P R}$ & & $r_{P S}$ & $r_{R S}$ \\
40 & 75 & & & & & $r_{S P S}$ & $r_{R S}$ \\
\hline 25 & 40 & $z$ & $z$ & & $z$ & & \\
25 & 50 & $r_{P S}+d_{P}^{o}$ & $r_{R S}+d_{R}^{o}$ & & & & \\
40 & 60 & $r_{S P}$ & $r_{S R}$ & $r_{P R}$ & & & \\
40 & 65 & $r_{S P S}$ & $r_{S R S}$ & $r_{P R}$ & $z$ & $r_{S P}$ & $r_{S R}$ \\
40 & 70 & $r_{S P S}$ & $r_{S R S}$ & $r_{P R}$ & & $r_{S P}$ & $r_{S R}$ \\
40 & 75 & & & & & $r_{S P S}$ & $r_{S R S}$
\end{tabular}

are not spontaneously formed in the phase separating mixture. Network-like structures are suppressed on similar grounds. Only rotationally symmetric stacks, i.e. the $r$ and $z$-stacks, are expected to be stable against the continuous deformations brought upon the fluid mixture by the shearing Couette cell. Interestingly, the simulations under shear also yield a structure that was not previously observed, namely an $r$ stack with three bands.

All two-banded $r$-stacks at the lower shear rate have the dense polymer or liquid crystal fluid at the inside and the less dense solvent on the outside. This distribution is in line with the theoretical predictions and the simulation results for the quiescent system. For the two- 
banded $r$-stacks at the higher shear rate, however, we observe that the order of the bands has reversed, with the solvent now consistently covering the inner wall and the fluid of chains occupying the outer wall. Three underlying mechanisms are hypothesized to explain this inversion of the steady state:

1. Following a thermodynamical line of thought, any system strives to minimize its free energy within the restrictions set by the externally imposed conditions. In the absence of shear, this free energy is dominated by the interfacial contributions, as discussed in Sections 4.3.1 and 4.3.2. The flow field $v_{\theta}(r)$ may be accounted for, in a casual approach inspired by statistical-mechanics, by extending the free energy with a kinetic term,

$$
F_{\text {kin }}=\frac{1}{2} \int_{V} \rho(\mathbf{r}) v_{\theta}^{2}(\mathbf{r}) d \mathbf{r}=\frac{1}{2} m \sum_{i} v_{i \theta}^{2}-\frac{1}{2} N k_{B} T .
$$

In the last step a Maxwell-Boltzmann distribution of $v_{i \theta}$ centered around $v_{\theta}\left(r_{i}\right)$ is assumed, to arrive at a computationally attractive expression which avoids the analytic solution of the flow fields of shear-thinning fluids in various stackings. The extended free energy may explain some transitions with varying drum velocity, as illustrated by the examples in Table 4.4, but the overall agreement with the simulations remains wanting.

2. Based on the minimum dissipation theorem in fluid dynamics [6], the steady state may be identified as the configuration requiring the least power by the rotating wall in the stationary state.

The power is calculated from the shear stress $\tau_{r \theta}$ on the inner wall, using the expressions derived in the appendix. The results in Table 4.4 suggest that this mechanism is becoming important at the high shear rate, especially in the larger simulation boxes, while there is little correlation at the low shear rate. Of course, for stationary walls the power is zero and the mechanism holds no predictive power.

3. As noted earlier, a system can become kinetically trapped during the phase separation process. The centrifugal forces experienced by the rotating mixture may then explain the preference of the denser polymer and liquid-crystal fluids to gyrate to the outer wall, with the less dense solvent moving to the inner wall. Once nascent metastable 
Table 4.4: Differences in free energies and shear powers for binary $P_{7}-S$ systems, comparing four stacks in three Couette geometries (with inner radius $R_{i}$ and outer radius $R_{o}$ ) at two angular velocities $\omega_{i}$ of the inner wall. The free energies are based on the interfacial free energy and augmented with a kinetic term accounting for the flow field, see Eq. (4.11). The power required to rotate the inner wall is calculated using the expressions derived in the Appendix. Within each row the lowest free energy and the lowest shear power are taken as the reference points for all entries in that row - these minima therefore appear as zeros in the table. The steady states obtained from the phase separation simulations of these systems, (see Table 4.3), are marked with an asterisk.

\begin{tabular}{cc|cccc|cccc} 
& & \multicolumn{4}{|c}{ Free energy $(\varepsilon)$} \\
$R_{i} / R_{O}$ & $\omega_{i} / \operatorname{rad} \tau^{-1}$ & $z$ & $r_{P S}$ & $r_{S P}$ & $r_{S P S}$ & $z$ & $r_{P S}$ & $r_{S P}$ & $r_{S P S}$ \\
\hline $25 / 40$ & $10^{-3}$ & $10^{1.5 *}$ & 0 & $10^{2.0}$ & $10^{3.1}$ & $10^{3.1 *}$ & $10^{2.1}$ & $10^{0.7}$ & 0 \\
$25 / 40$ & $10^{-2}$ & $0^{*}$ & $10^{1.9}$ & $10^{1.4}$ & $10^{3.1}$ & $10^{3.9 *}$ & $10^{2.5}$ & $10^{2.5}$ & 0 \\
$40 / 60$ & $10^{-3}$ & $10^{2.9}$ & $0^{*}$ & $10^{2.3}$ & $10^{3.3}$ & $10^{3.5}$ & $10^{2.8 *}$ & $10^{2.1}$ & 0 \\
$40 / 60$ & $10^{-2}$ & $10^{2.7}$ & $10^{2.4}$ & $0^{*}$ & $10^{3.2}$ & $10^{4.3}$ & $10^{3.3}$ & $10^{3.1 *}$ & 0 \\
$40 / 65$ & $10^{-3}$ & $10^{3.3}$ & $0^{*}$ & $10^{2.2}$ & $10^{3.1}$ & $10^{3.2}$ & $10^{2.6 *}$ & $10^{2.6}$ & 0 \\
$40 / 65$ & $10^{-2}$ & $10^{3.2}$ & $10^{2.6}$ & 0 & $10^{3.0 *}$ & $10^{4.1}$ & $10^{3.3}$ & $10^{3.0}$ & $0^{*}$
\end{tabular}

structure has formed, it will be difficult for the preceding two mechanisms to drive the system away from the metastable state to a state of minimum free energy or minimum work.

More generally, the three above mechanisms may be invoked to explain the steady states of the other systems as well, both under shear and at zero shear. The data in Table 4.2 through Table 4.4 suggest, however, that none of these mechanism describes all final states correctly. Most likely, the thermodynamic argument presides at zero and small shear rates, the flow argument becomes increasingly important with rising shear rates, and the trapping argument repeatedly frustrates these mechanisms by capturing the system in a long-lived sub-optimal state. 


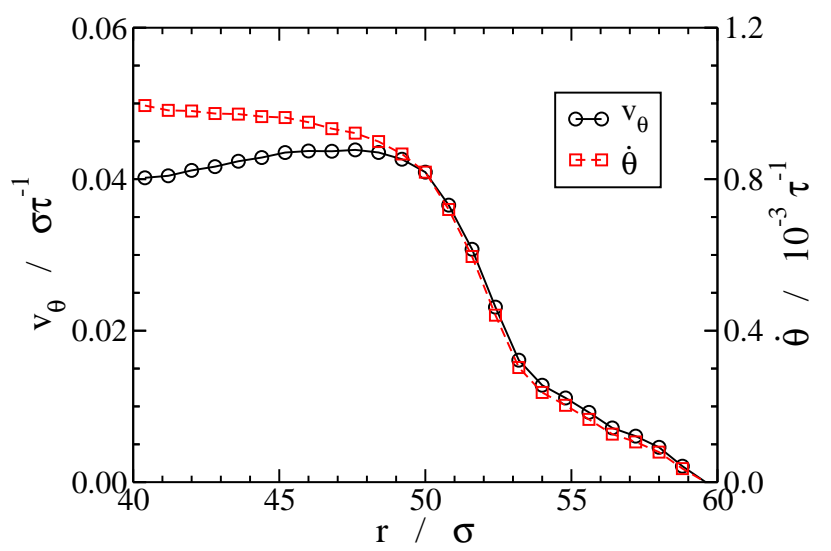

Figure 4.6: The flow profile $v_{\theta}(r)$ (solid line, left axis) and angular velocity profile $\dot{\theta}(r)=$ $v_{\theta}(r) / r$ (dashed line, right axis) for an $r$-stack with the rigid polymer $R_{7}$ on the inside and the solvent on the outside.

A typical flow profile $v_{\theta}(r)$ and the corresponding angular velocity distribution $\dot{\theta}(r)=$ $v_{\theta}(r) / r$ are shown in Fig. 4.6 for an $r$-stack with the polymers on the inside and the solvent on the outside. Because of the high viscosity of the polymeric fluid, this melt hardly flows under the applied shear and the azimuthal velocity $v_{\theta}$ even increases with $r$ in this section of the Couette cell. The angular velocity $\dot{\theta}$ slightly decays, confirming that the melt behaves as a viscous fluid rather than a plug flow. At the polymer-solvent interface, for $r \approx 52 \sigma$, the velocity profile shows a steep drop, indicative of a significant degree of slippage. Rod-solvent and even solvent-solvent [90] systems show similar hallmarks of slippage at their interfaces.

It might be argued that the partial non-stick boundary condition is exacerbated by the purely repulsive interactions between unlike particles, which weakens the mutual grip of the two fluids. The width of the decay region results from slight undulations in the interface, which is not perfectly smooth. In the solvent region of the Couette cell, beyond the slip layer, the flow profile continues to gradually decay until reaching zero at the outer wall. We note that at high shear rates a soliton-like perturbation appears at the interface, which appears to orbit the inner wall indefinitely [90]. 


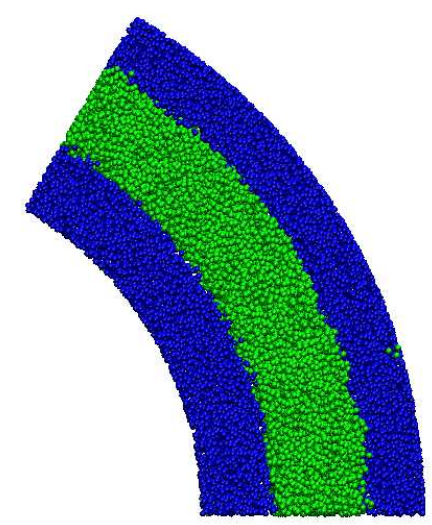

Figure 4.7: Top view of the steady state for a solvent (blue) - polymer $\left(P_{7}\right.$, green) mixture in a Couette cell of dimensions $R_{i}=40 \sigma$ and $R_{o}=65 \sigma$, with the inner wall rotating at $0.05 \mathrm{rad} / \tau$.

A number of simulations, especially at elevated drum velocities, yield $r$-stacks with three bands, see Fig. 4.7. These systems invariably consist of a central layer of polymer sandwiched between two layers of solvent. The location of the central band, which is virtually impermeable to the solvent particles, varies from simulation to simulation, with typically about 40 to $50 \%$ of the solvent particles caught in the inner band. It might be argued that these triple $r$-stacks are kinetically trapped, but their abundance in Table 4.3 suggests otherwise. The extra interfacial area discards the minimum free energy mechanism, leaving the minimum dissipation mechanism as the most likely explanation. A calculation of the power required to rotate the inner wall, see Table 4.4, supports this conclusion. Note that an equivalent power must be extracted by the thermostat in order for the system to reach a steady state. To ascertain the impact of a high shear rate and two slipping interfaces on the temperature in the system, we have calculated radial temperature profiles from the velocity distributions along $\hat{\mathbf{e}}_{r}$ and $\hat{\mathbf{e}}_{z}$, i.e. the two directions perpendicular to the shear flow. Even for a very high angular velocity $\omega_{i}=0.05 \mathrm{rad} / \tau^{-1}$ of the inner wall, the thermostat proves capable of maintaining a relatively constant temperature, varying from $1.02 k_{B} / \varepsilon$ near the walls and in the slip layers 
to $1.05 k_{B} / \varepsilon$ in the centre of the cell.

In several simulations a droplet of the denser chain phase is formed. Sometimes a single large droplet contains all chains, while in other cases the droplet is accompanied by an $r$ stack. All observed droplets under shear are attached to the outer wall, and are occasionally accompanied by an $r$-stack of chains covering the inner wall. At the higher shear rate, this ordering of the $r$-stack contrasts with the regular double banded $r$-stack at high shear rates and the triple banded $r$-stack, which both expose a solvent layer to the inner wall. The droplet gradually moves along the outer wall by the flow in the surrounding solvent. By tracking individual chains in the droplet, the droplet is found to slowly roll along the wall.

\subsubsection{Decomposition dynamics under shear}

In addition to its impact on the final stationary state, the shear also appears to promote the phase separation process. This acceleration is clearly recognizable in the snapshots of in Fig. 4.8, showing the progression of the decomposition at a fixed time lapse for a number of inner wall velocities. At zero and low shear rate the growing domains are significantly
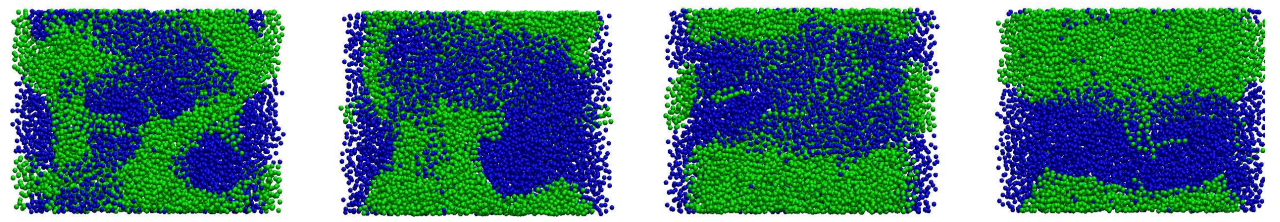

Figure 4.8: Snapshots of four phase separating polymer solvent-mixtures at $800 \tau$ after the quench, for inner wall angular velocities of $\omega_{i}=(0,1,10,50) \times 10^{-3} \mathrm{rad} / \tau$ (from left to right). These side views, with the drum axis vertically and the shear flow running from left to right, highlight the acceleration of the decomposition process under shear.

smaller than at the high shear rates, where the formation of $z$-stacks has nearly reached the steady state. Inspection of movies generated from the stored trajectories suggests that the non-uniform shear flow field assists the separation process both by bringing separate domains together and by pulling domains apart. The latter mechanism, which at first sight might appear to slow down the separation, advances the rupture of thin necks connecting large 
domains - these necks would have survived longer in the absence of shear - and thus also prevents the system from becoming trapped in an intermediate network configuration. It is impossible, however, to make a more quantitative assessment of the contributions of the various mechanisms to the overall acceleration. An acceleration of the phase separation under shear has also been reported in previous experimental and simulation studies [14,54]. The break-up of domains under shear is also expected to introduce a maximum attainable domain size, but this limit is apparently not reached under the current simulation conditions.

A final state of an $r$-stack coexisting with a droplet can be formed along two distinct kinetic pathways. In the first route a homogeneously mixed state evolves a cell-spanning structure akin to a $\theta$-stack, which subsequently is twisted, stretched and torn apart under the stresses imposed by the ongoing rotation of the inner wall. The fragment attached to the inner wall forms an $r$-stack, while the remainder at the outer wall compacts into a droplet. In the second route a mixed state appears to evolve into an triple-banded $r$-stack, consisting of a solvent layer between two polymer layers. The metastable outer layer eventually ruptures and contracts into a single droplet. 


\subsection{Conclusions}

The spinodal decompositions under shear of polymers and polymer liquid crystals solvated in a simple liquid have been studied by molecular dynamics simulations of a miniaturized Taylor-Couette rheometer. Most mixtures phase separate into radial, azimuthal or axial stacks reflecting the geometry of the confining walls, see Fig. 4.1, but we also observed a number of exceptions. The phase behaviour of the system is fairly complex as the steady state configuration is determined by a number of parameters, including the dimensions of the cell and the angular velocity of the rotating inner drum. We believe that the current simulations give a qualitatively realistic impression of the phase behaviour to be expected in microfluidic experiments on polymer-solvent mixtures, which constitutes an interesting experimental challenge.

The simulations strongly suggest that the equilibrium and non-equilibrium steady states formed by the phase separating mixtures are determined by a balance between two mechanisms, namely the minimisation of free energy and the minimisation of viscous dissipation. It is obvious that the thermodynamic mechanism rules in a quiescent mixture, implying that the preferred phase separated state is the configuration with the lowest total interfacial energy. The induced flow field at low angular velocity of the inner wall is already sufficiently strong to suppress the formation of $\theta$-stacks, yet the thermodynamic mechanism appears still capable of selecting the configuration with the lowest interfacial energy among the remaining allowed stacks, i.e. the rotational symmetric and therefore shear-resistant stacks. At high shear rates the hydrodynamic flow field emerges as the dominant factor, by preferentially establishing the configuration requiring the lowest work from the rotating drum wall in the steady state. These global trends are extracted from the simulation results, but are by no means conclusively proven at this stage. A number of simulations deviate from this model, e.g. the steady states with a droplet attached to a wall, which we currently attribute to kinetic trapping during phase separation. Nor is it clear at what shear rate the hydrodynamic mechanism takes precedence over the thermodynamic mechanism; the suppression of $\theta$-stacks at the lowest shear rate may be interpreted to suggest that the transition depends on the stack (or effectively, on the torque needed to shear the stack).

A second important property that surfaced during the simulations is the significant slippage of two fluids at their joint interface. The partial slip is clearly visible in the plots of the 
azimuthal velocity versus the radius, see Fig. 4.6, and is also manifest from visualisations of the simulations by following individual particles near the interface. Indications of slippage are even detectable at the macroscopic level, in the form of an additional third layer in the radial stacks at high shear rate. We speculate that the formation of this extra layer is instigated by the hydrodynamic mechanism outlined above, as the slippage at the thermodynamically unfavourable extra interface reduces the viscous dissipation in the system. Conversely, thermodynamic arguments may have prevented the formation of even more radial bands. One should keep in mind that the purely repulsive potential between unlike particles contributes to the slippage at the interface, and hence that a real fluid-fluid interface is expected to show a less pronounced sliding of the interfaces. The multiple radial bands in shear experiments are therefore more likely explained by incomplete phase separation and by shear-limited domain growth than by slip at the interfaces. 


\section{Appendix A: Shear stress $\tau_{r \theta}$ in a Couette geometry}

The relevant shear stress in the Couette geometry is $\tau_{r \theta}$, the stress acting along the azimuthal direction on a plane whose normal, points in the radial direction. In the steady state this stress will be a function of $r$ only. A conceptually appealing way of deriving an expression for $\tau_{r \theta}$ starts by looking at the axial component of the total angular momentum of all particles outside a coaxial cylinder of radius $r$,

$$
L_{z}(r)=\sum_{i} m_{i}\left(\mathbf{r}_{i} \times \mathbf{v}_{i}\right)_{z} \Theta\left(r_{i}-r\right),
$$

where $\Theta$ denotes the Heaviside step function. The time derivative of this angular momentum, i.e. the torque exerted by the interior of the cylinder on the exterior, reads as

$$
\begin{aligned}
\dot{L}_{z}(r) & =\sum_{i}\left(\mathbf{r}_{i} \times \mathbf{F}_{i}\right)_{z} \Theta\left(r_{i}-r\right)+\sum_{i} m_{i}\left(\mathbf{r}_{i} \times \mathbf{v}_{i}\right)_{z} \delta\left(r_{i}-r\right) \dot{r}_{i} \\
& =\sum_{i} \sum_{j}\left(\mathbf{r}_{i} \times \mathbf{F}_{i j}\right)_{z} \Theta\left(r_{i}-r\right) \Theta\left(r-r_{j}\right)+\sum_{i} m_{i}\left(\mathbf{r}_{i} \times \mathbf{v}_{i}\right)_{z} \boldsymbol{\delta}\left(r_{i}-r\right) \dot{r}_{i}
\end{aligned}
$$

In the second line we have expressed the total force $\mathbf{F}_{i}$ on particle $i$ as a sum of pair forces $\mathbf{F}_{i j}$, where $j$ lies either inside or outside the cylinder of radius $r$; in the latter case the pair force makes no contribution to the torque and can be omitted. The average torque is expressed mesoscopically as shear stress times lever arm integrated over the surface $S$ of the cylinder of height $h$,

$$
\left\langle\dot{L}_{z}(r)\right\rangle=\left(\int_{S} \mathbf{r} \times \tau \cdot d \mathbf{S}\right)_{z}=-2 \pi r^{2} h \tau_{r \theta}(r),
$$

where the stress matrix is assumed independent of $\theta$, in the last step. The power corresponding with this torque reads as

$$
P(r)=\int_{S} \mathbf{v} \cdot \tau \cdot d \mathbf{S}=2 \pi r h v_{\theta}(r) \tau_{r \theta}(r)
$$

in the stationary state, when the velocity field has converged to $\mathbf{v}(\mathbf{r})=v_{\theta}(r) \hat{\mathbf{e}}_{\theta}$ and $\tau$ has become rotation symmetric.

In the stationary state the axial angular momentum $L_{z}(r, \Delta r)$ of any concentric cylindrical shell with inner radius $r$ and thickness $\Delta r$ must be constant, hence its time derivative must vanish:

$$
0=\frac{d}{d t} L_{z}(r, \Delta r)=\frac{d}{d t}\left[L_{z}(r)-L_{z}(r+\Delta r)\right] .
$$


Consequently, the torque acting in every coaxial cylindrical plane must be identical and the product $r^{2} \tau_{r \theta}(r)$ will be independent of $r$. We take advantage of this property to reduce the noise level, by averaging the shear stress over all cylinders with radii between $r$ and $r+\Delta r$,

$$
\begin{aligned}
\overline{r^{2} \tau_{r \theta}(r)} & =-\frac{1}{2 \pi h} \frac{1}{\Delta r} \int_{r}^{r+\Delta r}\left\langle\dot{L}_{z}\left(r^{\prime}\right)\right\rangle d r^{\prime} \\
& =-\frac{1}{2 \pi h} \frac{1}{\Delta r}\left\langle\sum_{\substack{i \\
r \leq r_{i}<r+\Delta r}} m_{i} r_{i} v_{i \theta} v_{i r}+\sum_{\substack{i \\
r \leq r_{i}}} \sum_{\substack{j \\
r_{j} \leq r_{i} \\
r_{j} \leq r+\Delta r}} f_{i j}(r, r+\Delta r) r_{i}\left[\mathbf{F}_{i j} \cdot \hat{\mathbf{e}}_{\theta}\left(\mathbf{r}_{i}\right)\right]\right\rangle,
\end{aligned}
$$

where the weight factor is given by

$$
f_{i j}\left(r_{1}, r_{2}\right)=\int_{r_{1}}^{r_{2}} \Theta\left(r_{i}-r\right) \Theta\left(r-r_{j}\right) d r
$$

The only non-zero values to this integral are found when all three conditions in the doublesummations are met, i.e, $r_{i} \geq r_{1}, r_{j} \leq r_{2}$ and $r_{i}>r_{j}$, in which case

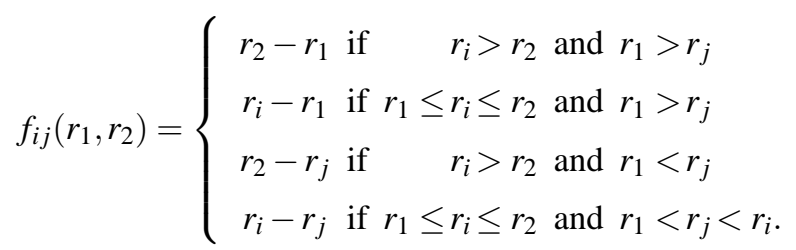

Simulations under shear, of both simple liquids and $r_{P S}$-stacks, confirm that the right hand side of Eq. (A.8) is indeed independent of $r$.

We end this appendix with three brief comments on the stress expression. i) A related expression has been used before to calculate stress profiles in flat geometries, for instance the pressure profile in a lipid bilayer [28], based on a more general derivation of the stress tensor by Schofield and Henderson [77]. Following analogies with the above derivation, one readily obtains the appropriate stress expressions from the time derivative of the total linear momentum of all particles at one side of a flat plane. ii) The change of the angular momentum $L_{z}(r)$, or the corresponding change of the linear momentum, is readily seen to require a summation over all pair forces acting between pairs on either side of the dividing plane. We therefore conclude that the dissipative and random pair forces introduced by the DPD thermostat also have to be included in the stress calculation, though the random forces are easily shown to average out to zero. This situation is in contrast to Brownian Dynamics 
simulations, where the friction and random forces are implemented as self-interactions of the particles and therefore do not contribute to the stress. iii) The above stress expressions, especially Eq. (A.2) and Eq. (A.8), contain a peculiar asymmetric treatment of the particles $i$ and $j$. In both expressions the pair force $\mathbf{F}_{i j}$ is projected on the $\hat{\mathbf{e}}_{\theta}$ direction at the position of particle $i$, subject to the restriction that $i$ is the outermost particle, $r_{i}>r_{j}$. An alternative derivation, based on the angular momentum of the particles inside a cylinder of radius $r$, even yields a final expression in which the pair-force projection is made with respect to the innermost particle. Both seemingly distinct expression yield identical results, however, since $\mathbf{r}_{i} \times \mathbf{F}_{i j}=\mathbf{r}_{j} \times \mathbf{F}_{i j}$ for pair forces acting along $\hat{\mathbf{r}}_{i j}$. Equation (4.5) is finally obtained by symmetrising the stress expression with respect to the particles $i$ and $j$, and by extending the average in Eq. (A.8) to include the entire Couette cell. 



\title{
Effect of confinement on the interfacial dynamics of binary liquid films
}

\begin{abstract}
We study the relaxation dynamics of capillary waves in the interface between two confined liquid layers by means of molecular dynamics simulations. We measure the auto-correlations of the interfacial fourier modes and find that the finite thickness of the liquid layers leads to a marked increase of the relaxation times as compared to the case of fluid layers of infinite depth. The simulation results are in good agreement with a theoretical first-order perturbation derivation which starts from the overdamped Stokes' equation. The theory also takes into account an interfacial friction, but the difference with no-slip interfacial conditions is small. When the walls are sheared, it is found that the relaxation times of modes perpendicular to the flow are unaffected. Modes along the flow direction are relatively unaffected as long as the equilibrium relaxation time is sufficiently short compared to the rate of deformation. We discuss consequences for experiments on thin layers, experiments on ultra-low surface tension fluids, as well as computer simulations.
\end{abstract}

\subsection{Introduction}

Phase separation is ubiquitous in nature. In liquids, or liquids dispersed with buoyant bodies such as colloids, polymers or surfactants, small differences between the mutual attractions of the various molecules are capable of separating like molecules from unlike molecules. Given enough time, such systems fully separate into macroscopically large domains, only limited 
by walls confining the system. The geometry of the interface between such macroscopically separated domains is generally determined by a combination of various interfacial tensions $\gamma_{i j}$ and the geometry of the confining walls. Although the interface between two phases appears to be smooth on macroscopic scales, thermal excitations cause the interface to be rough on microscopic scales. The broadening of an interface by these so-called capillary waves $[9,13,72]$ should be distinguished from its intrinsic width caused by partial mixing of the molecules of both phases [65]: for simple fluids interacting via van der Waals forces the local density of one species changes monotonically across the interface from its bulk liquid value to zero $[22,43,78]$. For ordinary molecular liquids, with surface tensions in the $\mathrm{mN} / \mathrm{m}$ range, the capillary roughness is relatively small, in the order of a few or tens of Ångstroms, and can be accessed experimentally by ellipsometry [7,61], X-ray scattering [23,60,75] and neutron $[39,55,76]$ reflectometry. Interestingly, in a recent series of papers $[1,18,73]$, Aarts and coworkers focused on colloid-polymer mixtures in which the interfacial tension between polymer lean and polymer rich phases is lowered to the $\mathrm{nN} / \mathrm{m}$ range. Because of this ultralow surface tension, the characteristic length and time scales of the interfacial fluctuations are such (micrometers and seconds, respectively) that they can be studied in real space by means of confocal microscopy.

An often used approximation in capillary wave theory $[9,13,72]$ is that the wavelength of the fluctuation under investigation is much smaller than the thickness of the liquid layer below the interface and, in case of two liquids, also much smaller than the thickness of the liquid layer above the interface. In many experimental situations this approximation is valid and greatly simplifies the theoretical analysis [62]. There are however situations in which the thickness of the liquid layer is of the same order of magnitude as the interfacial wavelength under consideration, or even smaller. For example, thin films with a thickness of (tens of) nanometers could be studied by means of X-ray scattering or neutron reflectometry $[39,76]$, or thin films with a thickness of hundreds of nanometers by light scattering. Moreover, as noticed above, characteristic lengths in the micrometer range occur in ultra-low surface tension fluids $[1,18,73]$. Finite size effects may become noticeable in the dynamics of such systems even when the liquid layers are as thick as a hundred micrometers. Simultaneously, rapid advances in the field of microfluidics enable the production of ever smaller systems, possibly containing phase separating fluids $[64,81]$. As the distances between walls in microfluidic 
devices decrease, the influence of finite-size effects on liquid-liquid interfacial fluctuations will continue to increase.

We expect that in most computer simulations of phase separated systems $[65,71,78$, $83,94,102]$ the dynamics of interfacial fluctuations are influenced by the finite thickness of the liquid layer(s) as well. Indeed, molecular dynamics simulations are usually limited to $10^{5}$ particles, which means that the simulation boxes are usually cubic, with fluid layers of a few nanometers thick. Although in simulation work much attention has been given to equilibrium properties of the interface, such as molecular organisation and structure and density profiles $[65,71,78,83,94]$, to the best of our knowledge no direct measurements have been reported of the dynamics of capillary interfacial fluctuations.

In this chapter we study, by means of particulate computer simulations, the dynamics of capillary interfacial fluctuations of a binary-liquid system with unlimited (periodic) area but finite depth. We will consider both quiescent and slowly sheared systems. For the small wavelike perturbations considered here, we can neglect the non-linear term in the general Navier-Stokes equation, but we will take into account the finite viscosity $\eta$ of the fluid. This was already done by Jeng et al. [40], but these authors focused on infinite depth systems. In fact, in this chapter we will focus on the overdamped limit, where viscosity dominates over inertia and the system can be considered to always be in a quasi-steady state. Also complementary to the work of Jeng et al. [40], we will take into account the effect of tangential friction $\beta$ between the liquid layers. In order to clearly expose the finite size effects, we consider here two liquids of equal density $\rho$, viscosity $\eta$ and depth $H$. In the equal density approximation the influence of gravitational acceleration $g$ can be ignored or, equivalently, the capillary length $\xi=\sqrt{\gamma / \Delta \rho g}$ is infinite. The above conditions have been chosen to match our simulations. We will compare the measured spectrum of relaxation times $\tau_{q}$ of the capillary modes of wave vector $q$ with a theoretical prediction:

$$
\tau_{q}=\frac{4 \eta}{\gamma q} \frac{\beta H\{\cosh q H \sinh q H+q H\}+\eta q H\left\{\cosh ^{2}(q H)+(q H)^{2}\right\}}{\beta H\left\{\sinh ^{2}(q H)-(q H)^{2}\right\}+\eta q H\{\cosh q H \sinh q H-q H\}},
$$

and find quantitative agreement. A derivation of this expression is given in the Appendices. The most prominent feature is a smooth crossover in the scaling of $\tau_{q}$, from $\tau_{q} \propto q^{-4}$ for small liquid layer depths $(q H \downarrow 0)$ to $\tau_{q} \propto q^{-1}$ for large liquid layer depths $(q H \rightarrow \infty)$. Each of the imposed conditions (overdamped motion, equal density, viscosity and liquid depth) 
may of course be relaxed, but will lead to more complicated theoretical expressions.

\subsection{Simulation set-up}

We use a simulation model similar to our previous work on phase separation $[89,90]$. The interactions between like particles are based on the Lennard-Jones (LJ) potential,

$$
U_{L J}\left(r_{i j}\right)=4 \varepsilon\left[\left(\frac{\sigma}{r_{i j}}\right)^{12}-\left(\frac{\sigma}{r_{i j}}\right)^{6}\right]
$$

where $\varepsilon$ and $\sigma$ are the strength and range respectively and $r_{i j}$ is the distance between particles $i$ and $j$. Both the potential and the derived force are smoothly truncated at the cut-off distance $2.5 \sigma$, to eliminate discontinuities at the latter distance. Unlike particles interact by the purely repulsive Weeks-Chandler-Andersen (WCA) potential, defined by $U_{W C A}(r)=U_{L J}+\varepsilon$ for $r \leq 2^{1 / 6} \sigma$ and $U_{W C A}(r)=0$ for larger separations. In our simulations, Newton's equations of motion are integrated numerically using the Verlet leap-frog algorithm [3] with a time step of $0.002 \tau$, where $\tau=\sqrt{\left(m \sigma^{2} / \varepsilon\right)}$ and $m$ is the mass of a particle. The temperature used in the simulations is $k T=1.0 \varepsilon$, where $k$ is Boltzmann's constant. A thermostat is used to sample an isothermal system. Many thermostats interfere with the creation of hydrodynamics flow fields, and therefore are not suited for the study of the interfacial fluctuations with boundaries as hydrodynamics might play a significant role in such systems. We have used the friction and random forces of dissipative particle dynamics (DPD) [29,35] to thermostat the system, as these forces are particularly designed to conserve local momentum, which is also the basis of the Navier-Stokes equation. In DPD the friction force $\mathbf{F}_{\text {fric }}$ and random force $\mathbf{F}_{\text {ran }}$ between a pair of particles separated by a distance $r$ within the cutoff distance $r_{c}$ is given by

$$
\begin{aligned}
\mathbf{F}_{\text {fric }} & =-\frac{\kappa^{2}}{2 k_{B} T}\left(1-\frac{r}{r_{c}}\right)^{2}(\hat{\mathbf{r}} \cdot \Delta \mathbf{v}) \hat{\mathbf{r}} \\
\mathbf{F}_{\text {ran }} & =\frac{\kappa}{\sqrt{\Delta t}}\left(1-\frac{r}{r_{c}}\right) \zeta \hat{\mathbf{r}}
\end{aligned}
$$

where $\kappa$ is the strength of the friction constant, which we set equal to $0.3 \varepsilon \tau^{1 / 2} \sigma^{-1}$ in this work, $\hat{\mathbf{r}}$ is the unit vector in the direction of the line joining the two particles, $\Delta \mathbf{v}$ is the velocity difference between the particle pair, and $\zeta$ is a random number with zero mean and unit variance. Compared to our previous work $[89,90]$, the value of $\kappa$ is chosen to 
be relatively small in order to avoid a too high viscosity of the fluid, as this would lead to extremely slow relaxation of the slowest interfacial modes. The geometry of our system is shown in Figure 5.1. These rectangular boxes are periodic in the $x$ and $y$ directions but a wall

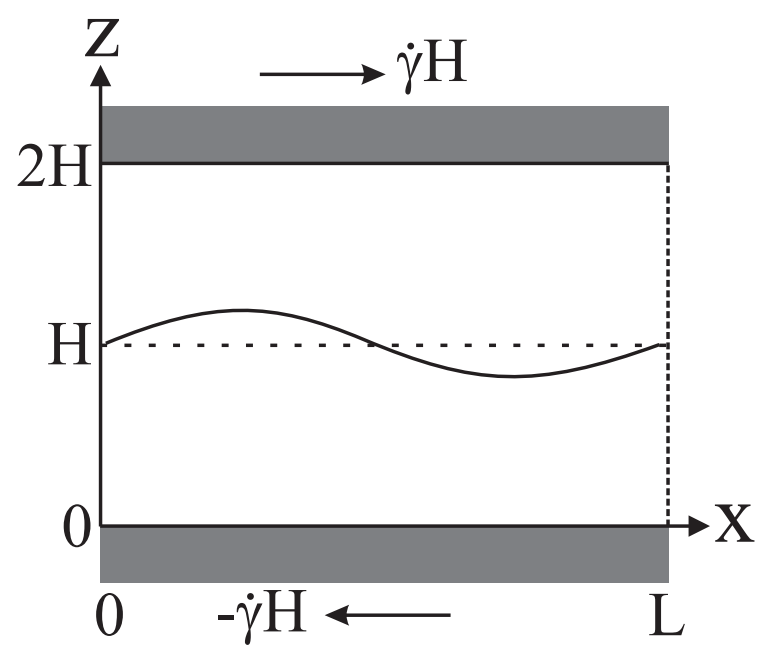

Figure 5.1: Geometry of the problem. Two equal amounts of fluid are enclosed between two walls at $z=0$ and $z=2 H$. The interface between the two liquids is on average at $z=H$. The walls are driven in the $x$-direction to produce an overall shear rate of $\dot{\gamma}$, i.e. the upper (lower) wall has a horizontal velocity of $(-) \dot{\gamma} H$. The system is periodic in the $x$ and $y$ directions with a period $L$.

is used in the $z$ direction to simulate the finite size effect and also to impose shear. A bounce back rule is applied to keep the particles in the boxes where velocities were modified after collision as $\mathbf{v}_{i} \mapsto-\mathbf{v}_{i}$. To impose a shear rate $\dot{\gamma}$ one of the walls is moved in the horizontal $x$-direction and all particles colliding with this wall are bounced back with an additional velocity component corresponding to that of the wall. The height of the box is fixed to $L_{z}=2 H=17.1 \sigma$ and the horizontal dimensions $L_{x}=L_{y}=L$ are varied between $L_{z}, 2 L_{z}$ and $4 L_{z}$. Note that $z$ is the direction normal to the fluid-fluid interface and that these choices enable us to study interfacial fluctuations at wavelengths larger than the depth of the fluid. The number of particles in the smallest box is $N=3500$ with a density of $\rho=0.7$. The number of particles in the other boxes are scaled accordingly. The boxes are equilibrated for 
$100 \tau$ before the actual samples are analyzed for results.

\subsection{Analysis of capillary waves}

To disentangle capillary waves from the intrinsic width of the interface, at regular time instances we divide the simulation box into $N_{x} \times N_{y} \times N_{z}$ cells. For every cell labeled by $(i, j, k)$ with $i \in\left(1, \ldots, N_{x}\right), j \in\left(1, \ldots, N_{y}\right)$, and $k \in\left(1, \ldots, N_{z}\right)$, we calculate the difference between the number of $A$ particles and the number of $B$ particles, $\Delta N(i, j, k)=N_{A}(i, j, k)-N_{B}(i, j, k)$. The height $h_{I F}(i, j)$ of the interface is then obtained in each column $(i, j)$ by least-squares fitting to a tangent hyperbolic profile:

$$
\Delta N^{\mathrm{fit}}(i, j, k)=a(i, j) \tanh \left[\frac{z(k)-h_{I F}(i, j)}{w(i, j)}\right]
$$

where $z(k)$ is the height of the centre of the cell with $z$-index $k$ and $\left(a, w, h_{I F}\right)$ are fit parameters: $a(i, j)$ is related to the bulk density of $A$ and $B$ particles, $w(i, j)$ is the intrinsic interfacial width, and $h_{I F}(i, j)$ is the height of the interface in column $(i, j)$. When the intrinsic interfacial width is very small and the interface is centred around $z=L_{z} / 2$, as is the case in our simulations, it is advantageous to choose an odd number of cells $N_{z}$ in the $z$-direction because this will lead to a more accurate sampling of the step-like profile. From the interfacial height $h_{I F}(i, j)$ we calculate its Fourier transform

$$
h_{\mathbf{q}}(t)=\frac{1}{N_{x} N_{y}} \sum_{i}^{N_{x}} \sum_{j}^{N_{y}} h_{I F}(i, j) \exp \left\{\mathrm{i}\left[q_{x} x(i)+q_{y} y(j)\right]\right\},
$$

where $(x(i), y(j))$ is the location of the centre of the column with indices $(i, j)$, and the wave vector $\mathbf{q}=q_{x} \hat{\mathbf{e}}_{x}+q_{y} \hat{\mathbf{e}}_{y}$ is chosen to be commensurate with the periodic box dimensions, i.e. $q_{x}=(2 \pi / L) n_{x}$ and $q_{y}=(2 \pi / L) n_{y}$, with $n_{x}$ and $n_{y}$ integer numbers. The amplitude of a capillary wave is then calculated as $\left\langle\left|h_{\mathbf{q}}\right|^{2}\right\rangle=\left\langle h_{\mathbf{q}} h_{\mathbf{q}}^{*}\right\rangle$, where the pointy brackets indicate averaging over different time instances. To determine the relaxation times $\tau_{q}$ we calculate the time correlation functions $\left\langle h_{\mathbf{q}}(t) h_{\mathbf{q}}^{*}(0)\right\rangle$.

In our simulations we choose the number of cells as $N_{x}=N_{y}=10$ and $N_{z}=7$ for the smallest box size. For larger boxes $N_{z}$ was kept the same, but $N_{x}$ and $N_{y}$ were scaled according to the box size. In the analysis the wave numbers were varied between $n_{x}=0, \ldots, 4$ and $n_{y}=0, \ldots, 4$. Higher wave numbers were not selected to avoid discretisation artefacts. 


\subsection{Results}

\subsubsection{Characterisation of the fluids}

In order to test our theoretical prediction Eq. (5.1), we first need to independently determine the viscosity, surface tension and interfacial friction of our fluids. We assume these quantities are independent of wave vector $\mathbf{q}$ in the range of wave lengths studied.

For the viscosity $\eta$ we consider a box containing only A particles, with periodic boundaries in all directions. The viscosity is obtained by slowly shearing using Lees-Edwards sliding-brick boundary conditions [3] and dividing measured shear stress by applied shear rate. The result of this measurement is $\eta=1.23 k T \tau / \sigma^{3}$.

The surface tension $\gamma$ is determined in a box, periodic in all directions, containing a layer of A particles and a layer of B particles. The interface normals are along the $z$-axis. In such a configuration the surface tension is related to the difference between the average normal $P_{z z}$ and average tangential $\left(P_{x x}+P_{y y}\right) / 2$ pressure [48]:

$$
\gamma=\frac{L_{z}}{2}\left[P_{z z}-\frac{1}{2}\left(P_{x x}+P_{y y}\right)\right]
$$

where the factor 2 arises from the fact that there are two interfaces within each periodic box. The result of this measurement is $\gamma=1.23 k T / \sigma^{2}$.

Two fluid layers may have (partial) tangential slip, resulting in a tangential velocity difference $\Delta v$ between the two surfaces. The tangential force per unit area exerted by the surface of one fluid on the surface of the other fluid is given, phenomenologically, by the product of slip velocity and interfacial friction coefficient $\beta$ :

$$
F^{\beta}=\beta \Delta v
$$

Suppose we apply an overall shear rate $\dot{\gamma}$ to a system containing layers of fluid $A$ and $B$. If there is a finite amount of slip, the effective shear rate $b$ within the fluid layers will be lower than $\dot{\gamma}$. From a simple consideration of force balances, knowing the viscosity $\eta$ of the fluid layers, the following relation can be derived:

$$
\beta=\frac{\eta}{L_{z}} \frac{b}{\dot{\gamma}-b} .
$$

We have measured the effective shear rates $b$ in the liquid layers for shear rates between 
$0.01 \tau^{-1}$ and $0.05 \tau^{-1}$ and found $b / \dot{\gamma}=0.76$, independent of applied shear rate $\dot{\gamma}$. For our model we therefore estimate an interfacial friction coefficient $\beta=0.228 k T \tau / \sigma^{4}$.

\subsubsection{Amplitudes of interfacial fluctuations}

As long as the amplitudes of the interface fluctuations are small the interfacial free energy can be approximated as

$$
\begin{aligned}
A & =\gamma \int_{0}^{L_{x}} \mathrm{~d} x \int_{0}^{L_{y}} \mathrm{~d} y \sqrt{1+\left(\frac{\partial h_{I F}}{\partial x}\right)^{2}+\left(\frac{\partial h_{I F}}{\partial y}\right)^{2}} \\
& \approx \gamma L_{x} L_{y}\left(1+\sum_{\mathbf{q}}^{\prime}\left|h_{\mathbf{q}}\right|^{2} q^{2}\right)
\end{aligned}
$$

where the prime indicates summation over independent $h_{\mathbf{q}}$ in two adjacent quadrants of the $\mathbf{q}$ vector space. Since the free energy contributions are of quadratic form, and each $h_{\mathbf{q}}$ is complex and therefore effectively contributes to two modes, the equipartition theorem predicts the following mean square amplitudes:

$$
\left\langle\left|h_{\mathbf{q}}\right|^{2}\right\rangle=\frac{k_{B} T}{\gamma L_{x} L_{y} q^{2}}
$$

In Figure 5.2 we show the mode amplitudes $\left\langle\left|h_{\mathbf{q}}\right|^{2}\right\rangle$ for various box sizes $L$ and wave-vectors $\mathbf{q}$ as measured in equilibrium simulations. Fig. 5.2 also shows the theoretical prediction Eq. (5.12), using the independently determined surface tension $\gamma$. All data clearly coincide with the theoretical curve, indicating that our cell-based procedure to measure capillary interface fluctuations is valid.

\subsubsection{Dynamics of interfacial fluctuations}

We now proceed to measure the relaxation times $\tau_{q}$ of the various capillary wave modes in equilibrium simulations. According to Onsager's regression hypothesis [66] the relaxation dynamics may be obtained from the auto-correlations $\left\langle h_{\mathbf{q}}(t) h_{\mathbf{q}}^{*}(0)\right\rangle$. In Appendix 5.5 we derive an equation of motion for $h_{\mathbf{q}}$ for overdamped capillary waves, which is valid up to first order in $h_{\mathbf{q}}$ and at times beyond the ballistic regime. This equation of motion predicts an 


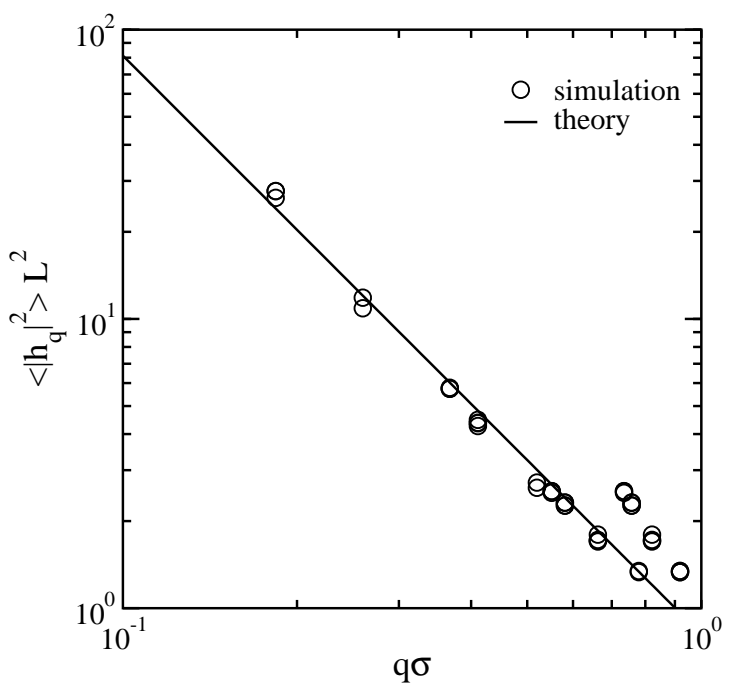

Figure 5.2: Amplitudes of capillary waves. Mean-square amplitudes $\left\langle\left|h_{\mathbf{q}}\right|^{2}\right\rangle$ of various fourier modes of the interfacial height are determined and multiplied by the bare surface area $L^{2}$ (symbols). Good agreement with the theoretical prediction Eq. (5.12) is found (solid line).

exponential decay of the auto-correlation:

$$
\frac{\left\langle h_{\mathbf{q}}(t) h_{\mathbf{q}}^{*}(0)\right\rangle}{\left\langle\left|h_{\mathbf{q}}\right|^{2}\right\rangle}=\exp \left(-t / \tau_{q}\right) .
$$

In the ballistic regime at early times, for reasons of time-symmetry the derivative of $\left\langle h_{\mathbf{q}}(t) h_{\mathbf{q}}^{*}(0)\right\rangle$ must be zero near $t=0$. The relaxation times $\tau_{q}$ are therefore extracted by fitting to an exponential decay in the region where the normalised auto-correlation decays from 0.75 to 0.05 , see Fig. 5.3. The lower limit serves to exclude the noisy data that occurs at larger correlation times. Figure 5.4 is the main result of this chapter. We plot the measured relaxation times as a function of $q$ for various box sizes and wave vectors. Clearly, our results are in the transitional region from a $\tau_{q} \propto q^{-4}$ to a $\tau_{q} \propto q^{-1}$ scaling. We also plot the theoretical prediction, Eq. (5.1) (solid line), using the independently measured values of viscosity $\eta$, surface tension $\gamma$ and interfacial friction coefficient $\beta$, and find good agreement between simulation and theory. Comparing our relaxation times with the prediction for infinitely high liquid lay- 


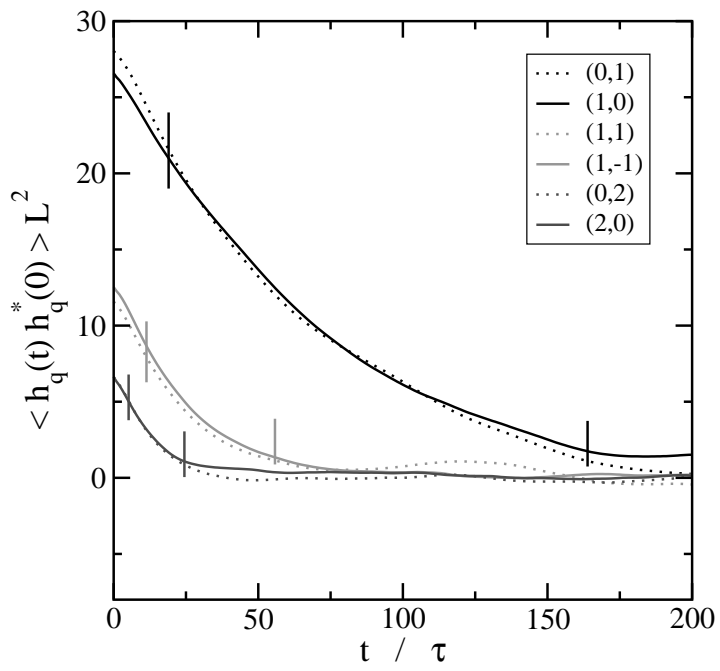

Figure 5.3: Dynamics of capillary waves. Autocorrelations $\left\langle h_{\mathbf{q}}(t) h_{\mathbf{q}}^{*}(0)\right\rangle$ of fourier modes of the interfacial height multiplied by the bare surface area $L^{2}$ for the case $L=4 H$. The wave vectors are chosen according to $\mathbf{q}=(2 \pi / L)\left(n_{x}, n_{y}\right)$, where $\left(n_{x}, n_{y}\right)$ are pairs of integer numbers (see legend). Relaxation times $\tau_{q}$ are estimated by fitting the regions between the vertical lines to an exponential decay.

ers, $\tau_{q}=4 \eta /(\gamma q)$ (dashed line in Fig. 5.4), we observe that inclusion of finite size effects is essential for the prediction of the dynamics of capillary modes when $q H$ is of order 1 or smaller.

The often used approximation of no-slip boundaries between the liquid layers, corresponding to the case $\beta \rightarrow \infty$, is plotted as the dot-dashed line in Fig. 5.4. The differences between predictions with finite and infinite $\beta$ are small. Our measurements are not accurate enough to distinguish between these two cases. We remind the reader that our fluids have purely repulsive interactions between unlike particles. This may lead to much more interfacial slip than with real fluids which usually have some attraction, whether strong or weak, even between unlike particles. The difference with the $\beta \rightarrow \infty$ limit may therefore even be smaller for real fluids. 


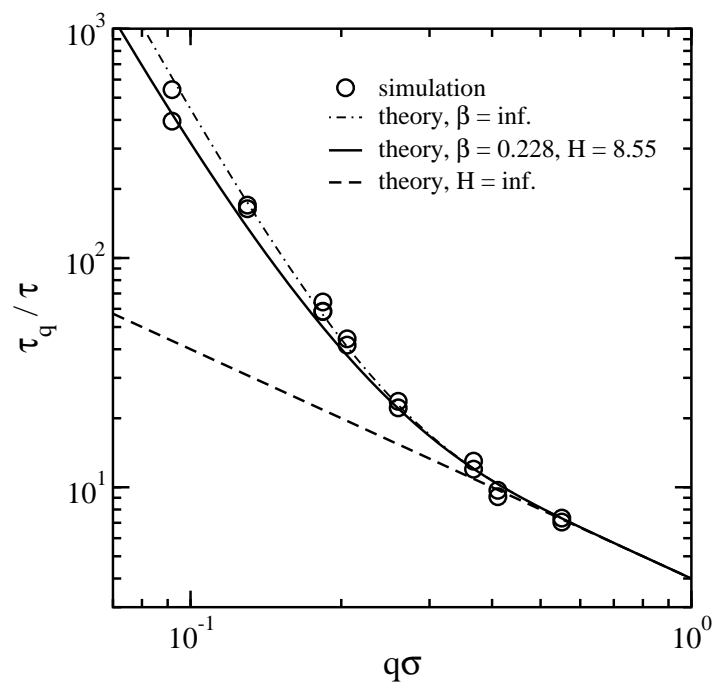

Figure 5.4: Relaxation times of capillary waves. Simulation results (symbols) are from systems with $H=7.55 \sigma$ and $L=2 H, L=4 H$ and $L=8 H$. The theoretical prediction Eq. (5.1), using independently measured values of $\eta, \gamma$ and $\beta$, is given by the solid line. Two limits of the theoretical prediction are also shown: the case of infinite interfacial friction $\beta$ (dot-dashed line) and the case of infinite liquid layer depth $H$ (dashed line).

\subsubsection{Influence of shear flow}

We finally study the influence of shear flow on the relaxation dynamics of capillary waves. The first order theory presented in the Appendices predicts relaxation times $\tau_{q}$ which are independent of the applied shear rate $\dot{\gamma}$. We should be careful of this result, however, because inertial and other higher order effects may become dominant at higher shear rates. A correct theoretical treatment of this case would therefore involve including inertial terms in the hydrodynamic equations and deriving equations of motion for $h_{q}$ up to second order. This is a topic of future research. Here we set ourselves the more modest task of studying the extent to which equilibrium predictions still apply under shear flow.

To a good approximation the flow and relaxation in the vorticity (y) direction are decoupled from those in the flow $(x)$ direction. We therefore expect essentially unchanged relaxation times for modes perpendicular to the flow direction (i.e. wave vectors with component 
$q_{x}=0$ ). This is tested in Fig. 5.5 where we show the height autocorrelation of the mode given by $\left(n_{x}=0, n_{y}=1\right)$ at various shear rates. We confirm that in all cases the relaxation dynamics are similar, even at relatively high shear rates. The relaxation times of modes with

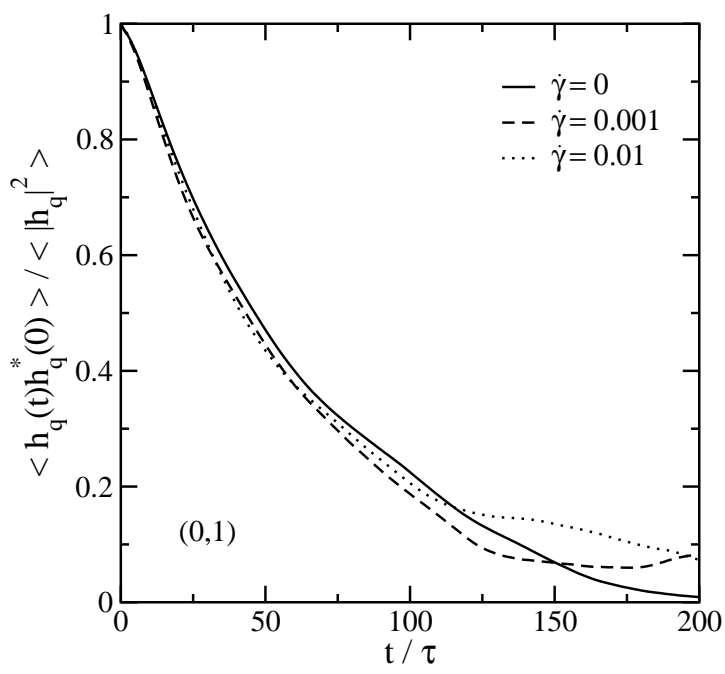

Figure 5.5: Normalised relaxation dynamics of capillary waves at different shear rates $\dot{\gamma}$ for the system with $L=4 H$. In all cases the wave vector is equal to $\mathbf{q}=(2 \pi / L)(0,1)$, which is perpendicular to the direction of flow.

a non-zero component in the flow direction can be severely affected however. In Fig. 5.6 we present the height autocorrelation of the mode given by $\left(n_{x}=1, n_{y}=0\right)$ at various shear rates. Indeed, at small shear rates the relaxation dynamics is similar to the equilibrium case, next there is a small increase in relaxation time, while finally the relaxation is accelerated by the flow at higher shear rates. We hypothesise that the relaxation time of a mode with $x$-component $q_{x}$ is not essentially altered by shear flow if this mode has relaxed sufficiently far before the shear flow has had a chance of deforming it. This means that we expect that $\tau_{q}$ is not changed if $\dot{\gamma} \tau_{q_{x}}^{e q} \ll 1$, where $\tau_{q_{x}}^{e q}$ is the relaxation time, in equilibrium, of a mode $\left(q_{x}, 0\right)$. We test our hypothesis in Fig. 5.7. This figure shows the spectrum of equilibrium relaxation times $\tau_{q_{x}}^{e q}$ along the vertical axis, repeated along the horizontal axis for each inverse shear rate $\dot{\gamma}^{-1}$ studied. Crosses indicate modes which are relatively unaffected by shear flow (here 


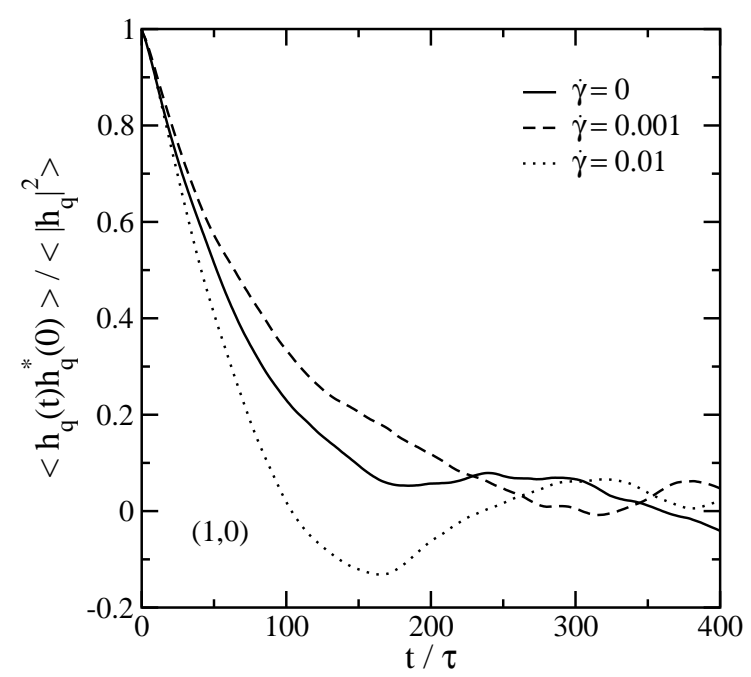

Figure 5.6: Normalised relaxation dynamics of capillary waves at different shear rates $\dot{\gamma}$ for the system with $L=4 H$. In all cases the wave vector is equal to $\mathbf{q}=(2 \pi / L)(1,0)$, which is in the direction of flow.

we choose the rather arbitrary criterium of less than $20 \%$ change), whereas circles indicate modes which are affected (more than $20 \%$ change in relaxation time) by the shear flow. On a $\log -\log$ scale, the boundary between these two regions can be described by $\left(\dot{\gamma} \tau_{q_{x}}^{e q}\right)^{\alpha}$, with the exponent in the range $\alpha=(1.0 \pm 0.3)$. If we may assume that the boundary is indeed given by our hypothesised exponent of $\alpha=1$, we can quantify the " $\ll 1$ " in our hypothesis: the relaxation times are relatively $(<20 \%)$ unaffected as long as $\dot{\gamma} \tau_{q_{x}}^{e q}<0.2$.

\subsection{Conclusions}

We have studied the overdamped relaxation dynamics of capillary waves between two liquid layers enclosed by walls. The finite depths of the liquid layers lead to a large increase of the relaxation times of the larger wave length modes. We have compared our simulation results with a theoretical calculation, and found good agreement. It was also found that the inclusion of an interfacial friction $\beta$ in the theory only slightly changes the predictions of 


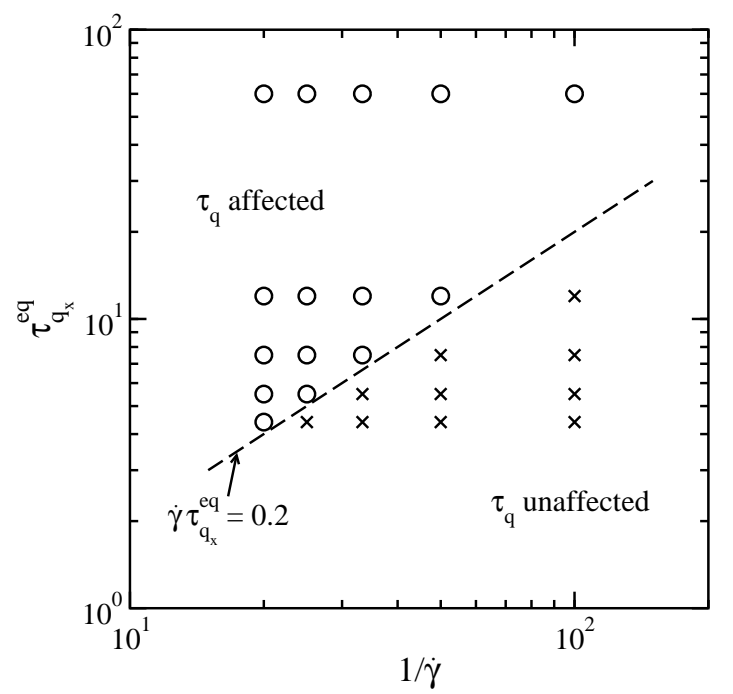

Figure 5.7: Non-equilibrium phase diagram showing which modes are affected by shear flow in the $L=4 H$ system. The spectrum of equilibrium relaxation times $\tau_{q_{x}}^{e q}$ of modes $\left(q_{x}, 0\right)$ is given along the vertical axis, repeated for each inverse shear rate $1 / \dot{\gamma}$ studied. Crosses indicate modes which are relatively unaffected by shear flow (relaxation time changes by less than 20\% compared to equilibrium), whereas circles indicate modes which are affected (more than $20 \%$ change).

the relaxation times. Our measurements are not accurate enough to distinguish between the actual and infinite interfacial friction. Finally, we have shown that the relaxation dynamics is unaffected by shear flow for modes in the vorticity direction. Modes $\mathbf{q}$ with a non-zero component in the flow direction are also relatively unaffected as long as $\dot{\gamma} \tau_{q_{x}}^{e q}<0.2$, where $\tau_{q_{x}}^{e q}$ is the relaxation time in equilibrium of a mode with wave vector $\left(q_{x}, 0\right)$.

These results are of importance to experiments that probe the interfacial dynamics between thin (tens of nanometers) films or ordinary liquids, experiments on ultra-low surface tension fluids at larger length scales (tens of micrometers), as well as applications of microfluidics. Moreover, the results are relevant for almost all simulation studies on the dynamics of liquid-liquid interfaces. Because in most simulation works the box dimensions are chosen rather similar along the different coordinate axes, the product $q H$ is of order 1 for the largest 
wave length that fits in the simulated box. Specifically, for a cubic box $(q H=\pi)$ the deviations from the $q H \rightarrow \infty$ limit may be small but distinguishable, while for larger $L / H$ ratios the deviations increase strongly, as shown in this work.

\section{Appendix A: Flow field between solid walls and a fluc- tuating interface}

In this Appendix we will solve the flow-field $\mathbf{u}$ and pressure field $P$ between solid walls and a fluctuating interface. Figure 5.1 shows the geometry of the problem: we consider a two-phase liquid system enclosed between walls at $z=0$ and $z=2 H$. These walls are driven in the $x$ direction to produce an overall shear rate of $\dot{\gamma}$ and the system is periodic in the $x$-direction with a period $L$. In the analysis we will focus on the $x$ - and $z$-coordinates, assuming without loss of generality of our results that the pressure and flow-fields are translationally invariant in the $y$-direction.

The interface (IF) fluctuates around $z=H$. Let us describe its coordinates by a Fourier cosine series:

$$
h_{I F}(x, t)=H+\sum_{q \neq 0} h_{q}(t) \cos q x
$$

where $q=2 \pi n / L$, with $n$ a positive integer, in order to comply with our periodic setup. In the overdamped limit viscosity dominates over inertia, the system is always in quasi-equilibrium, and we can consider the relaxation of a standing wave described by the time dependence of the coefficients $h_{q}(t)$. Inclusion of sine terms in the above equation (to account for traveling waves) will then not be necessary. Our aim in this section is to solve the quasi-stationary Stokes' equations

$$
\begin{aligned}
\eta \nabla^{2} \mathbf{u} & =\boldsymbol{\nabla} P \\
\boldsymbol{\nabla} \cdot \mathbf{u} & =0
\end{aligned}
$$

for the upper and lower fluids in the presence of the walls and the fluctuating interface described by the amplitudes $h_{q}(t)$. In the next section we will consider force balances at the interface, from which we will be able to derive an equation of motion for $h_{q}$ and determine the spectrum of relaxation times. 
Let us first focus on the lower fluid between $z=0$ and $z=h_{I F}$. Periodic boundary conditions apply at $x=0$ and $x=L$, and the wall at $z=0$ forms a no-slip boundary. The boundary conditions at the interface require some more thought. Because the lower fluid is confined below the interface, the vertical $(z-)$ velocity of the fluid at the interface is determined by the time evolution of the amplitudes $h_{q}(t)$ :

$$
u_{z}\left(x, H+\sum_{q} h_{q} \cos q x\right)=\frac{\partial h_{I F}}{\partial t}=\sum_{q} \dot{h}_{q}(t) \cos q x,
$$

where the dot above $h_{q}$ indicates differentiation with respect to time. If the amplitudes $h_{q}$ are small we may approximate this, up to first order in $h_{q}$, as the vertical velocity at $z=H$ :

$$
u_{z}(x, H)=\sum_{q} \dot{h}_{q}(t) \cos q x-\frac{\partial u_{z}}{\partial z} \sum_{q} h_{q} \cos q x \approx \sum_{q} \dot{h}_{q}(t) \cos q x .
$$

In summary, we have the following boundary conditions:

$$
\begin{aligned}
u_{x}(0, z) & =u_{x}(L, z) \\
u_{z}(0, z) & =u_{z}(L, z) \\
u_{x}(x, 0) & =-\dot{\gamma} H \\
u_{z}(x, 0) & =0 \\
u_{z}(x, H) & =\sum_{q} \dot{h}_{q} \cos q x .
\end{aligned}
$$

Notice that we have not specified a boundary condition for the horizontal $(x-)$ velocity at $z=H$. We cannot specify it because we want to include the possibility of slip between the two fluids. We will be able to fully solve the set of equations in the next section, when we balance tangential hydrodynamic forces on the interface with the interfacial slip forces.

Taking the divergence of Eq. A.2, the pressure field is found to obey Laplace's equation $\nabla^{2} P(x, z)=0$. Already taking into account the periodic boundaries in the the $x$-direction, Eqs. (A.6) and (A.7), this suggests the following homogeneous solution to the flow-field in the $x$ direction:

$$
\begin{aligned}
u_{x}(x, z)=\sum_{q \neq 0} & \quad\left\{\left(A_{q 1}+B_{q 1} z\right) \sin q x \sinh q z+\left(A_{q 2}+B_{q 2} z\right) \cos q x \sinh q z\right. \\
+ & \left.\left(A_{q 3}+B_{q 3} z\right) \sin q x \cosh q z+\left(A_{q 4}+B_{q 4} z\right) \cos q x \cosh q z\right\} \\
+ & a+b z .
\end{aligned}
$$


A similar expression may be written for $u_{z}(x, z)$ with other coefficients $\tilde{A}_{q 1}, \ldots, \tilde{A}_{q 4}, \tilde{B}_{q 1}, \ldots, \tilde{B}_{q 4}$, $\tilde{a}$ and $\tilde{b}$. Inserting the no-slip boundary condition Eq. (A.8) yields

$$
A_{q 3}=0, \quad A_{q 4}=0, \quad a=-\dot{\gamma} H .
$$

Here and in the following an expression like $A_{q 3}=0$ means that the coefficients $A_{q 3}$ are zero for all allowed $q \neq 0$. The boundary condition Eq. (A.9) yields

$$
\tilde{A}_{q 3}=0, \quad \tilde{A}_{q 4}=0, \quad \tilde{a}=0 .
$$

The interface boundary conditions, Eq. (A.10), yields

$$
\begin{aligned}
\left(\tilde{A}_{q 1}+\tilde{B}_{q 1} H\right) \sinh q H+\tilde{B}_{q 3} H \cosh q H & =0 \\
\left(\tilde{A}_{q 2}+\tilde{B}_{q 2} H\right) \sinh q H+\tilde{B}_{q 4} H \cosh q H & =\dot{h}_{q} \\
\tilde{b} & =0 .
\end{aligned}
$$

Finally, using the in-compressibility of the fluid, Eq. (A.3), we find the following relations for the coefficients of the flow-field:

$$
\begin{array}{lll}
\tilde{B}_{q 1}=B_{q 4} & q \tilde{A}_{q 1}=-B_{q 2} & q A_{q 1}=B_{q 3} \\
\tilde{B}_{q 2}=-B_{q 3} & q \tilde{A}_{q 2}=B_{q 1} & q A_{q 2}=B_{q 4} \\
\tilde{B}_{q 3}=B_{q 2} & q \tilde{A}_{q 3}=0 & q A_{q 3}=0 \\
\tilde{B}_{q 4}=-B_{q 1} & q \tilde{A}_{q 4}=0 & q A_{q 4}=0 \\
\tilde{b}=0 & \tilde{a}=0 & a=-\dot{\gamma} H .
\end{array}
$$

So we come to the conclusion that we can express the flow field solely in terms of the unknown (for now) coefficients $b, B_{q 1}$ and $B_{q 2}$. For ease of notation we will also keep using the coefficients $B_{q 3}$ and $B_{q 4}$, but note that $B_{q 4}$ can be written in terms of $B_{q 2}$ by Eqs. (A.14) and (A.17) and that $B_{q 3}$ can be written in terms of $B_{q 1}$ by Eqs. (A.15) and (A.17).

In summary, the flow-field of the lower fluid is given by

$$
\begin{gathered}
u_{x}(x, z)=-\dot{\gamma} H+b z+\sum_{q \neq 0}\left(\frac{B_{q 3}}{q}+B_{q 1} z\right) \sin q x \sinh q z+\left(\frac{B_{q 4}}{q}+B_{q 2} z\right) \cos q x \sinh q z \\
+B_{q 3} z \sin q x \cosh q z+B_{q 4} z \cos q x \cosh q z \\
u_{z}(x, z)=\sum_{q \neq 0}\left(-\frac{B_{q 2}}{q}+B_{q 4} z\right) \sin q x \sinh q z+\left(\frac{B_{q 1}}{q}-B_{q 3} z\right) \cos q x \sinh q z \\
+B_{q 2} z \sin q x \cosh q z-B_{q 1} z \cos q x \cosh q z .
\end{gathered}
$$


Knowing the flow field, it is a simple task to calculate the pressure field from Eq. (A.2):

$$
\begin{aligned}
P(x, z)=P_{0}+2 \eta \sum_{q \neq 0} \quad & \left\{B_{q 4} \sin q x \sinh q z-B_{q 3} \cos q x \sinh q z\right. \\
& \left.+B_{q 2} \sin q x \cosh q z-B_{q 1} \cos q x \cosh q z\right\},
\end{aligned}
$$

where $P_{0}$ is a reference pressure (the average pressure in absence of interfacial fluctuations).

Next we focus on the upper fluid between $z=h_{I F}$ and $z=2 H$. If we transform to coordinates $\xi=x$ and $\zeta=2 H-z$, the boundary conditions in these coordinates are given by

$$
\begin{aligned}
u_{\xi}(0, \zeta) & =u_{\xi}(L, \zeta) \\
u_{\zeta}(0, \zeta) & =u_{\zeta}(L, \zeta) \\
u_{\xi}(\xi, 0) & =+\dot{\gamma} H \\
u_{\zeta}(\xi, 0) & =0 \\
u_{\zeta}(\xi, H) & =-\sum_{q} \dot{h}_{q} \cos q \xi
\end{aligned}
$$

The solution to Stokes' equations (A.2) and (A.3) are then the same as for the lower fluid, only with $h_{q}$ replaced by $-h_{q}$ and $\dot{\gamma}$ replaced by $-\dot{\gamma}$. To distinguish the flow fields, we will label the coefficients which go with the flow-field of the upper fluid with primes, i.e. $b^{\prime}$ and $B_{q 1}^{\prime}, \ldots, B_{q 4}^{\prime}$. At this point we will make no assumptions about the relation between the coefficients $B_{q i}^{\prime}$ and $B_{q j}$.

\section{Appendix B: Force balances}

In this Appendix we will use the velocity and pressure fields derived in Appendix 5.5 to calculate the hydrodynamic stresses on the interface between the two fluids. Perpendicular to the interface the hydrodynamic stresses must be balanced by the surface tension. Parallel to the interface the difference between the hydrodynamic stresses is given by the interfacial slip force.

The hydrodynamic force per unit area on the interface due to the fluid below the interface is given by

$$
\mathbf{F}^{h}=-\boldsymbol{\sigma} \cdot \hat{\mathbf{n}}
$$


where $\boldsymbol{\sigma}$ is the stress tensor in the lower fluid close to the interface. Its components are given by

$$
\sigma_{\alpha \beta}=\eta\left\{\frac{\partial u_{\alpha}}{\partial x_{\beta}}+\frac{\partial u_{\beta}}{\partial x_{\alpha}}\right\}-\delta_{\alpha \beta} P,
$$

and $\hat{\mathbf{n}}$ is a unit vector normal to the interface. Up to first order in $h_{q}$, the latter is given by

$$
\hat{\mathbf{n}}(x)=\left(\sum_{q} h_{q} q \sin q x, 1\right)^{T} .
$$

We will also need the unit vector tangential to the interface, which up to first order in $h_{q}$ is given by

$$
\hat{\mathbf{t}}(x)=\left(1,-\sum_{q} h_{q} q \sin q x\right)^{T} .
$$

Using Eqs. (A.18) - (A.20), we find that the components of the hydrodynamic force perpendicular and parallel to the interface are given, up to first order in $h_{q}$, by

$$
\begin{aligned}
F_{\perp}^{h}(x)= & -\hat{\mathbf{n}}^{T} \cdot \boldsymbol{\sigma} \cdot \hat{\mathbf{n}} \\
= & P_{0}+2 \eta \sum_{q \neq 0}\left\{b h_{q} q \sin q x-B_{q 2} q H \sin q x \sinh q H+B_{q 1} q H \cos q x \sinh q H\right. \\
\quad & \left.\quad\left(B_{q 2}-B_{q 4} q H\right) \sin q x \cosh q H-\left(B_{q 1}-B_{q 3} q H\right) \cos q x \cosh q H\right\},
\end{aligned}
$$

Let us now consider the elastic force on the interface due to surface tension. If the coordinates of the interface are given by $h_{I F}(x, y, t)$, the interfacial free energy is given by Eq. (5.10). Up to first order, a local change in the interfacial height at $(x, y)$ leads to a change in interfacial free energy given by

$$
\frac{\delta A}{\delta h_{I F}(x, y, t)}=-\gamma\left(\frac{\partial^{2} h_{I F}}{\partial x^{2}}+\frac{\partial^{2} h_{I F}}{\partial y^{2}}\right) .
$$


Using the Fourier cosine series, Eq. (A.1), we find a local free energy change of

$$
\frac{\delta A}{\delta h_{I F}(x, y, t)}=\gamma \sum_{q \neq 0} h_{q} q^{2} \cos q x .
$$

The elastic force (per unit area) due to the surface tension $\gamma$ is therefore given up to first order in $h_{q}$ by

$$
\mathbf{F}^{e}=\left(0,-\gamma \sum_{q} h_{q} q^{2} \cos q x\right)^{T} .
$$

At any time and at any location along the interface the total force in the normal direction must be zero:

$$
F_{\perp}^{h}-F_{\perp}^{h \prime}+F_{\perp}^{e}=0
$$

where the prime in $F_{\perp}^{h \prime}$ indicates that the hydrodynamic force originates from the upper fluid. As argued at the end of Appendix 5.5, this can be found by replacing in Eq. (B.5) all $h_{q}$ by $-h_{q}$ and all coefficients by primed versions (the minus sign arises in the equation above because the $\zeta$-direction is opposite the $z$-direction). From Eq. (B.10) we derive for each $q$ up to first order in $h_{q}$ :

$$
\gamma h_{q} q^{2}=2 \eta\left\{\left(B_{q 1}-B_{q 1}^{\prime}\right)(q H \sinh q H-\cosh q H)+\left(B_{q 3}-B_{q 3}^{\prime}\right) q H \cosh q H\right\}
$$

Let us next consider the forces in the tangential direction. If the upper and lower fluids slip past each other, they will experience a friction force given by

$$
\mathbf{F}^{\beta}=\beta\left(\mathbf{u}\left(x, H^{+}\right)-\mathbf{u}\left(x, H^{-}\right)\right) \cdot \hat{\mathbf{t}} \hat{\mathbf{t}},
$$

where $\beta$ is the friction coefficient per unit area, $\mathbf{u}\left(x, H^{+}\right)$is the velocity of the upper fluid at the interface and $\mathbf{u}\left(x, H^{-}\right)$is the velocity of the lower fluid at the interface. At the interface, the tangential hydrodynamic force due to the lower liquid is exactly cancelled by the friction force, $F_{\|}^{h}+F_{\|}^{\beta}=0$. Similarly the tangential hydrodynamic force due to the upper liquid is exactly cancelled by the friction force, $F_{\|}^{h \prime}-F_{\|}^{\beta}=0$. Adding respectively subtracting these two expressions, we find that at any time and at any location along the interface the following balances must hold in the tangential direction:

$$
\begin{aligned}
F_{\|}^{h}+F_{\|}^{h \prime} & =0 \\
F_{\|}^{h}-F_{\|}^{h \prime}+2 F_{\|}^{\beta} & =0 .
\end{aligned}
$$


From the first equation we find that $B_{q i}^{\prime}=-B_{q i}(i=1, \ldots, 4)$, as could have been guessed from symmetry. From the second equation we derive (for each $q$ ) up to first order in $h_{q}$ :

$$
\begin{aligned}
0= & \left(B_{q 3}-B_{q 3}^{\prime}\right)\{\eta q(q H \sinh q H+\cosh q H)+\beta(\sinh q H+q H \cosh q H)\} \\
& +\left(B_{q 1}-B_{q 1}^{\prime}\right)\{\eta q q H \cosh q H+\beta q H \sinh q H\},
\end{aligned}
$$

as well as

$$
b=\dot{\gamma} \frac{1}{1+\frac{\eta}{2 \beta H}},
$$

in agreement with Eq. (5.9). In deriving the last equation we have used the fact that the effective shear rates are equal in the upper and lower fluids, i.e. $b^{\prime}=-b$. Eq. (B.16) shows that the effective shear rate in the fluid goes asymptotically towards the applied shear rate $\dot{\gamma}$ as the interfacial friction $\beta$ tends to infinity (no-slip shear flow). In the other limit, when the interfacial friction tends to zero, the effective shear rate in the fluid goes to zero (corresponding to two plug flows in opposite directions).

Although we now know that $B_{q 1}^{\prime}=-B_{q 1}$ and $B_{q 3}^{\prime}=-B_{q 3}$, from Eqs. (B.11) and (B.15) it becomes apparent that only the differences $B_{q 1}-B_{q 1}^{\prime}$ and $B_{q 3}-B_{q 3}^{\prime}$ are required to find the relaxation times of the modes $q$. Subtracting Eq. (A.15) from the equivalent expression for the upper fluid, and multiplying by $q$, we find for each $q$

$$
2 q \dot{h_{q}}=\left(B_{q 1}-B_{q 1}^{\prime}\right)\{\sinh q H-q H \cosh q H\}-\left(B_{q 3}-B_{q 3}^{\prime}\right) q H \sinh q H .
$$

From equations (B.11), (B.15) and (B.17) we derive, after some algebra, the following differential equation for $h_{q}$ :

$$
\frac{\dot{h}_{q}}{h_{q}}=-\frac{\gamma q}{4 \eta} \frac{\beta\left\{\sinh ^{2}(q H)-(q H)^{2}\right\}+\eta q\{\cosh q H \sinh q H-q H\}}{\beta\{\cosh q H \sinh q H+q H\}+\eta q\left\{\cosh ^{2}(q H)+(q H)^{2}\right\}} .
$$

Because the right-hand-side of the above equation is a negative constant for each $q$, we have derived that each mode in a macroscopically applied perturbation of the interface will decay exponentially (at least within our overdamped and first order approach) to zero. Onsager's regression hypothesis states that the regression of microscopic thermal fluctuations at equilibrium follows the macroscopic law of relaxation [66]. We therefore predict that the auto correlations of the Fourier modes of the capillary (thermal) interface fluctuations are given up to first order by

$$
\left\langle h_{q}(t) h_{q}(0)\right\rangle=\left\langle h_{q}^{2}\right\rangle \exp \left(-t / \tau_{q}\right)
$$


where $\left\langle h_{q}^{2}\right\rangle$ is the mean-square amplitude of mode $q$ and the relaxation time $\tau_{q}$ is given by

$$
\tau_{q}=\frac{4 \eta}{\gamma q} \frac{\beta H\{\cosh q H \sinh q H+q H\}+\eta q H\left\{\cosh ^{2}(q H)+(q H)^{2}\right\}}{\beta H\left\{\sinh ^{2}(q H)-(q H)^{2}\right\}+\eta q H\{\cosh q H \sinh q H-q H\}} .
$$

Several limits may be identified.

- When the fluid height $H$ is much larger than the wavelength of the fluctuation of interest, the actual value of the friction coefficient $\beta$ becomes irrelevant. In such a case we have

$$
\lim _{q H \rightarrow \infty} \tau_{q}=\frac{4 \eta}{\gamma q}
$$

This expression is in agreement with the result of Jeng et al. [40] for overdamped liquid-liquid interface fluctuations.

- When the fluid height $H$ is much smaller than the wavelength of the fluctuation of interest, and the friction coefficient is sufficiently large, we have

$$
\lim _{q H \downarrow 0 \beta H / \eta \rightarrow \infty} \lim _{q}=\frac{24 \eta}{\gamma q^{4} H^{3}} .
$$

- Finally, when the fluid height $H$ is much smaller than the wavelength of the fluctuation of interest, and the friction coefficient is negligible, we have

$$
\lim _{q H \downarrow 0 \beta H / \eta \downarrow 0} \lim _{q}=\frac{6 \eta}{\gamma q^{4} H^{3}} .
$$

For two thin layers of fluid, according to the last two equations, we find that the relaxation times of fluctuations of the interface can change by a factor of four, depending the strength of the interfacial friction. 


\section{Conclusions and Outlook}

In this thesis we report on molecular dynamics simulations of phase separation of simple and complex binary liquids in sheared and non-sheared systems. The separation of milk into liquid whey and solid curd is a very common example of phase separation observed in daily life. The phenomenon finds its application in various fields of science and technology, ranging from metals, semiconductors, superconductors to simple and complex fluids such as polymers, surfactants, colloids, emulsions and biological materials. The principles driving the phenomenon of phase separation are very fundamental in nature, i.e. interaction difference between like and unlike molecules, making it an interesting problem to study. The aim of our study is to enhance the understanding of the dynamics and rheology of the phenomenon of phase separation.

The most important property in the study of phase separation dynamics is the scaling of the domain size with time. The various exponents of the domain growth rate represent different stages of phase separation, in which different evolution mechanisms dominate. In chapter 2, we simulate the early stage of phase separation and show, for the first time in simulations, the corresponding domain growth exponent to be $1 / 3$, which corresponds to the diffusion mechanism. We further observe a temperature dependent transition from the diffusive to the hydrodynamic (viscous) growth regime, characterised by a growth exponent of unity. At high temperatures the critical slowing down plays a very important role and is responsible for delaying the onset of collective hydrodynamic behaviour.

To resemble the experimental simulations more closely and to study the effect of nonlinear shear, we study phase separation (chapter 3) in a Couette cell - a Couette cell is a geometry with fluid confined between two concentric cylinders. In non-sheared cases the final equilibrium state obtained by simulations was as predicted by the minimum free en- 
ergy calculations. In sheared simulations, starting from a well-mixed configuration, at low shear rate clear separated stacks form, while at higher shear rate transient elongated patterns emerge. The transition takes place where the rate of shear deformation is of the same order as the time scale for the transition from diffusive to viscous growth. When starting from fully separated configurations, different starting configurations show different behaviors on application of shear. Stacks in the gradient direction (interface normal points along shear direction) are inherently unstable under shear and the configuration break easily, even at a low shear, to form another stable configuration. Stacks in the flow direction (interface normal points along shear gradient direction) form at the interface a heap, moving with an angular velocity lower than that of the interface. Stacks in the vorticity direction (interface normal points in axial direction) are stable for the range of applied shears in our simulations.

We extend our investigation in chapter 4 to more complex liquids and study the phase separation in dynamically asymmetric polymer/solvent and liquid-crystal/solvent mixtures (Chapter 4). We examine both sheared and non-sheared cases. At lower shear rates, the free energy dominates and the mixtures relax to achieve their lowest free energy configurations. However at higher shear rates, viscous dissipation becomes a determining factor of the final stable configuration. A very interesting three band pattern is observed in cases of polymer/solvent and liquid-crystal/solvent mixture. This pattern is counter-intuitive because free energy arguments are against two interfaces. But the slip at both interfaces between polymer and solvent makes this structure attractive from a dissipation point of view. We also show that the stiffness of the liquid-crystals is responsible for the slow separation of liquidcrystal/solvent mixtures, as it prolongs the entangled regime, specially in case of longer chain molecules. Systems with long chains often are dynamically trapped in a local free energy minimum and stay there for a long time without change.

In Chapter 5, we focus on the effect of boundaries on the capillary waves in nano-films. Capillary waves are generated at the liquid interfaces due to thermal fluctuations. We measure the amplitude of the modes of interface fluctuations, and observe an inverse quadratic dependence on the wave-number, which is in agreement with the theory. Most of the previous studies discuss the interfacial fluctuations with boundaries at infinity, however we show by measuring the auto-correlations of the interfacial modes that close-by boundaries increase the relaxation times significantly. We also theoretically analyze Stokes' equation with a first 
order perturbation and show that the simulation results are in excellent agreement with the theory. Further, we include the effect of interfacial slip in the generalised theory, and show that in our simulations the difference between slip and no-slip is insignificant. In cases where we shear the system, we observe that the modes perpendicular to the flow direction are not affected by the shear. The modes parallel to the flow direction are unaffected as long as the rate of deformation is lower than the relaxation time of the mode and a change from over-damped to under-damped behaviour as the shear rate is increased.

As we have shown, even simple models for phase separation do remarkably good job in describing early stages of pattern formation observed in daily life. Simulation of late stages of phase separation by molecular dynamics is computationally demanding. A coarsegrained model, representing correct static and dynamic properties for fluids, would be useful in capturing the physics involved in the phenomenon of phase separation. However, the verification of various patterns, observed in our simulations, is still left as a challenge for experimentalists. We believe our simulation results will serve as an excellent guide to study phase separation in more complex liquids, under sheared and in confined geometries. 



\section{Samenvatting}

In dit proefschrift beschrijven we moleculaire dynamica simulaties van fasescheiding in simpele en complexe binaire vloeistoffen in stilstaande systemen en in een afschuifstroming. Het stremmen van melk in vloeibare wei en vaste wrongel is een bekend voorbeeld van fasescheiding in het dagelijks leven. Het verschijnsel vindt zijn toepassing in verschillende gebieden van wetenschap en technologie, variërend van metalen, halfgeleiders en supergeleiders tot simpele en complexe vloeistoffen zoals polymeren, oppervlakte-actieve stoffen, colloïden, emulsies en biologische materialen. De principes die aan fasescheiding ten grondslag liggen zijn erg fundamenteel van aard, namelijk interactieverschillen tussen gelijke en ongelijke moleculen, waardoor het een erg interessant probleem is om te bestuderen. Het doel van ons onderzoek is om een beter begrip te vormen van de dynamica en reologie van het verschijnsel fasescheiding.

De belangrijkste eigenschap in de studie van fasescheidingsdynamica is het schalen van de domeingroottes in de tijd. De verscheidene exponenten van de domeingroeisnelheid kenmerken verschillende stadia van fasescheiding, waarbij telkens een ander groeimechanisme overheerst. In hoofdstuk 2 simuleren we het eerste stadium van fasescheiding en vinden, voor de eerste keer in simulaties, de bijbehorende domeingroei-exponent van $1 / 3$, in overeenstemming met een diffusief mechanisme. Ook zien we een temperatuurafhankelijke overgang van het diffusieve naar het hydrodynamische (viskeuze) groeiregime, gekarakteriseerd door een groei-exponent van één. Bij hoge temperaturen speelt de kritische vertraging een belangrijke rol door het uitstellen van de start van collectief hydrodynamisch gedrag.

Om dichter bij de experimentele situatie aan te sluiten en om het effect te onderzoeken van een niet-lineaire afschuifstroming, bestuderen we in hoofdstuk 3 fasescheiding in een Couette cel een Couette cel is een geometrie met vloeistof ingesloten tussen twee concentrische cylinders. In niet-afgestroomde situaties is de uiteindelijke evenwichtstoestand van de simulaties in overeenstemming met voorspellingen van minimale vrije-energie berekeningen.In afgeschoven simulaties, beginnend met een goed gemengde toestand, ontstaat bij lage afschuifstroomsnelheid een duidelijk gescheiden stapeling, terwijl bij hoge afschuifstroomsnelheid kortstondig een gerekt patroon verschijnt. De overgang vindt plaats als de 
afschuifstroomsnelheid van dezelfde orde is als de tijdschaal van de overgang van diffusieve naar viskeuze groei. Als begonnen wordt met een volledig gescheiden toestand blijken verschillende starttoestanden tot verschillend gedrag te leiden onder een aangelegde afschuifstroming. Stapelingen in de gradiëntrichting (de grensvlaknormaal wijst langs de afschuifstromingsrichting) zijn inherent onstabiel onder een afschuifstroming en de toestand breekt makkelijk op, zelfs onder een lage afschuifstroomsnelheid, om een andere stabiele toestand te vormen. Stapelingen in de stromingsrichting (de grensvlaknormaal wijst langs de afschuifstromingsgradiënt) vormen aan het grensvlak een bobbel, die beweegt met een hoeksnelheid kleiner dan die van het grensvlak. Stapelingen in de wervelrichting (grensvlaknormaal wijst in de axiale richting) zijn stabiel voor het gebruikte bereik van afschuifstroomsnelheden in de simulaties.

We breiden ons onderzoek in hoofdstuk 4 uit naar complexere vloeistoffen en bestuderen fasescheiding in dynamisch-asymmetrische polymeer/vloeistof en vloeibaar-kristal/vloeistof mengsels. We bekijken zowel afgeschoven als niet-afgeschoven situaties. Bij lage afschuifstroomsnelheden domineert de vrije-energie en relaxeert het systeem naar toestanden met de laagste vrije-energie. Daarentegen bij hoge afschuifstroomsnelheden wordt viskeuze dissipatie een dominante factor voor de uiteindelijke stabiele toestand. Een erg interessant drie-banden patroon wordt gezien in mengsels van polymeren met vloeistof en van vloeibare kristallen met vloeistof. Dit patroon is tegen-intuïtief omdat vrije-energie argumenten tegen twee grensvlakken zijn. Maar de slip aan beide grensvlakken tussen polymeer en vloeistof maakt deze structuur wel aantrekkelijk vanuit een minimale-dissipatie gezichtspunt. We laten ook zien dat de stijfheid van de vloeibare kristallen verantwoordelijk is voor de trage scheiding van vloeibaar-kristal/vloeistof mengsels, omdat daardoor het verknopingsregime verlengd wordt, in het bijzonder voor lange ketenvormige moleculen. Systemen met lange ketens komen vaak vast te zitten in een lokaal vrije-energie minimum waar ze lange tijd in verblijven zonder te veranderen.

In hoofdstuk 5 kijken we naar het effect van wanden op de capillaire golven in nanofilms. Capillaire golven worden gegenereerd aan vloeistofgrensvlakken door thermische fluctuaties. We meten de amplitudes van de grensvlakfluctuatiemodi, en vinden een omgekeerdkwadratische afhankelijkheid met het golfgetal, hetgeen in overeenstemming is met de theorie. De meeste voorgaande studies beschrijven de grensvlakfluctuaties voor begrenzende 
wanden op oneindig, maar wij laten zien door het berekenen van de autocorrelaties van de grensvlakmodi dat nabije wanden de relaxatietijden aanzienlijk verhogen. We analyseren ook analytisch de Stokes-vergelijking met een eerste orde storing en laten zien dat de simulatieresultaten in goede overeenstemming zijn met de theorie. Vervolgens nemen we daarbij de effecten van grensvlakslip mee in de gegeneraliseerde theorie, en laten zien dat in onze simulaties het verschil tussen slip en geen-slip niet belangrijk is. In gevallen waar we het systeem afschuiven zien we dat modi die loodrecht op de stroomrichting staan niet beïnvloed worden door de afschuifstroming. De modi parallel aan de stroomrichting zijn ongewijzigd zolang de afschuifstroomsnelheid lager is dan de relaxatietijd van die modi en veranderen van overdempt naar onderdempt gedrag als de afschuifstroomsnelheid wordt verhoogd.

Zoals we hebben laten zien kunnen zelfs eenvoudige modellen voor fasescheiding een opvallend goede beschrijving geven van het eerste stadium van patroonformatie in het dagelijks leven. Simulaties van de latere stadia met moleculaire dynamica is computationeel veeleisend. Een grof-korrelig model, dat de statische en dynamische eigenschappen van vloeistoffen correct representeert, zou nuttig zijn bij het vangen van de fysica achter het verschijnsel fasescheiding. Echter, de verificatie van de verschillende patronen uit onze simulaties vormt een uitdaging voor experimentatoren. We verwachten dat onze simulatieresultaten als een goede leidraad kunnen dienen bij het bestuderen van complexe vloeistoffen, in een afschuifstroming en in besloten geometriën. 



\section{Bibliography}

[1] D. G. A. L. Aarts, M. Schmidt, and H. N. W. Lekkerkerker. Direct visual observation of thermal capillary waves. Science, 304(5672):847-850, May 2004.

[2] F. J. Alexander, S. Chen, and D. W. Grunau. Hydrodynamic spinodal decomposition - growth-kinetics and scaling functions. Physical Review B, 48(1):634-637, July 1993.

[3] M. P. Allen and D. J. Tildesley. Computer Simulation of Liquids. Oxford University Press, Oxford, U. K., 1987.

[4] C. Appert, J. F. Olson, D. H. Rothman, and S. Zaleski. Phase-separation in a 3-dimensional, 2-phase, hydrodynamic lattice-gas. Journal Of Statistical Physics, 81(1-2):181-197, October 1995.

[5] J. H. Aubert. Structural coarsening of demixed polymer-solutions. Macromolecules, 23(5):1446-1452, March 1990.

[6] G. K. Batchelor. An Introduction to Fluid Dynamics, pages 227-228. Cambridge University Press, Cambridge, U. K., 2002.

[7] D. Beaglehole. Capillary-wave and intrinsic thicknesses of the surface of a simple liquid. Physical Review Letters, 58(14):1434-1436, April 1987.

[8] A. J. Bray. Theory of phase-ordering kinetics. Advances In Physics, 43(3):357-459, May 1994.

[9] F. P. Buff, R. A. Lovett, and F. Stillinger. Interfacial density profile for fluids in critical region. Physical Review Letters, 15(15):621-\&, 1965.

[10] M. Cappelezzo, C. A. Capellari, S. H. Pezzin, and L. A. F. Coelho. Stokes-einstein relation for pure simple fluids. Journal Of Chemical Physics, 126(22):224516, June 2007.

[11] C. K. Chan, F. Perrot, and D. Beysens. Experimental-study and model simulation of spinodal decomposition in a binary mixture under shear. Physical Review A, 43(4):1826-1839, February 1991.

[12] Y. C. Chou and W. I. Goldburg. Phase-separation and coalescence in critically quenched isobutyric-acidwater and 2,6-lutidine-water mixtures. Physical Review A, 20(5):2105-2113, 1979.

[13] M. W. Cole. Width of surface layer of liquid he4. Physical Review A, 1(6):1838-\&, 1970.

[14] F. Corberi, G. Gunnella, and A. Lamura. Two-scale competition in phase separation with shear. Physical Review Letters, 83(20):4057-4060, November 1999.

[15] P. V. Coveney and K. E. Novik. Computer simulations of domain growth and phase separation in twodimensional binary immiscible fluids using dissipative particle dynamics. Physical Review E, 54(5):51345141, November 1996.

[16] W. K. den Otter and J. H. R. Clarke. Simulation of polymers by dissipative particle dynamics. In M. J. Kotelyanskii and D. N. Theodorou, editors, Simulation methods for polymers, pages 559-574. Marcel Dekker publishers, New York, 2004. 
[17] W. K. den Otter, S. A. Shkulipa, and W. J. Briels. Buckling and persistence length of an amphiphilic w orm from molecular dynamics simulations. Journal Of Chemical Physics, 119(4):2363-2368, July 2003.

[18] D. Derks, D. G. A. L. Aarts, D. Bonn, H. N. W. Lekkerkerker, and A. Imhof. Suppression of thermally excited capillary waves by shear flow. Physical Review Letters, 97(3):038301, July 2006.

[19] J. K. G. Dhont and W. J. Briels. Isotropic-nematic spinodal decomposition kinetics. Physical Review E, 72:031404, 2006.

[20] M. Doi and A. Onuki. Dynamic coupling between stress and composition in polymer-solutions and blends. Journal De Physique Ii, 2(8):1631-1656, August 1992.

[21] M. K. Endoh, M. Takenaka, and T. Hashimoto. Effects of shear flow on a semidilute polymer solution under phase-separating condition. Polymer, 47(20):7271-7281, September 2006.

[22] R. Evans, R. J. F. L. Decarvalho, J. R. Henderson, and D. C. Hoyle. Asymptotic decay of correlations in liquids and their mixtures. Journal Of Chemical Physics, 100(1):591-603, January 1994.

[23] C. Fradin, A. Braslau, D. Luzet, D. Smilgies, M. Alba, N. Boudet, K. Mecke, and J. Daillant. Reduction in the surface energy of liquid interfaces at short length scales. Nature, 403(6772):871-874, February 2000.

[24] D. Frenkel and B. Smit. Understanding molecular simulations. From algorithms to applications. Academic press, San Diego, CA, 2 edition, 2002.

[25] H. Furukawa. Dynamic-scaling theory for phase-separating unmixing mixtures - growth-rates of droplets and scaling properties of auto-correlation functions. Physica A, 123(2-3):497-515, 1984.

[26] H. Furukawa. A dynamic scaling assumption for phase-separation. Advances In Physics, 34(6):703-750, November 1985.

[27] H. Furukawa. Effect of inertia on droplet growth in a fluid. Physical Review A, 31(2):1103-1108, 1985.

[28] R. Goetz and R. Lipowsky. Comoputer simulations of bilayer membranes: self-assembly and interfacial tension. Journal Of Chemical Physics, 108:7397-7409, 1998.

[29] R. D. Groot and P. B. Warren. Dissipative particle dynamics: Bridging the gap between atomistic and mesoscopic simulation. Journal Of Chemical Physics, 107(11):4423-4435, September 1997.

[30] J. D. Gunton, M. San Miguel, and P. S. Sahni. xxx. In C. Domb and J. L. Lebowitz, editors, Phase transitions and critical phenomena, volume 8, page 267. Academic, New York, 1983.

[31] J. Harting, G. Giupponi, and P. V. Coveney. Structural transitions and arrest of domain growth in sheared binary immiscible fluids and microemulsions. Physical Review E, 75(4):041504, April 2007.

[32] T. Hashimoto and T. Kume. Butterfly light-scattering pattern in shear-enhanced concentration fluctuations in polymer-solutions and anomaly at high shear rates. Journal Of The Physical Society Of Japan, 61(6):18391843, June 1992.

[33] T. Hashimoto, T. Takebe, and K. Asakawa. Phase-transition of polymer mixtures under simple shear-flow. Physica A, 194(1-4):338-351, March 1993.

[34] P. C. Hohenberg and B. I. Halperin. Theory of dynamic critical phenomena. Reviews Of Modern Physics, 49(3):435-479, 1977. 
[35] P. J. Hoogerbrugge and J. M. V. A. Koelman. Simulating microscopic hydrodynamic phenomena with dissipative particle dynamics. Europhysics Letters, 19(3):155-160, June 1992.

[36] J. S. Huang, W. I. Goldburg, and A. W. Bjerkaas. Study of phase separation in a critical binary-liquid mixture - spinodal decomposition. Physical Review Letters, 32(17):921-923, 1974.

[37] W. Humphrey, A. Dalke, and K. Schulten. Vmd: visual molecular dynamics. J. Mol. Graph., 14:33-38, 1996.

[38] T. Imaeda, A. Furukawa, and A. Onuki. Viscoelastic phase separation in shear flow. Physical Review E, 70(5):051503, November 2004.

[39] U. Jeng, L. Esibov, M. L. Crow, and A. Steyerl. Neutron reflectometry study on an interface of octane and d2o at low surfactant (c(10)e(4)) concentration. Physica B, 221(1-4):168-173, April 1996.

[40] U. S. Jeng, L. Esibov, L. Crow, and A. Steyerl. Viscosity effect on capillary waves at liquid interfaces. Journal Of Physics-Condensed Matter, 10(23):4955-4962, June 1998.

[41] S. I. Jury, P. Bladon, S. Krishna, and M. E. Cates. Tests of dynamical scaling in three-dimensional spinodal decomposition. Physical Review E, 59(3):R2535-R2538, March 1999.

[42] H. Kabrede and R. Hentschke. Spinodal decomposition in a 3d lennard-jones system. Physica A-Statistical Mechanics And Its Applications, 361(2):485-493, March 2006.

[43] M. H. Kalos, J. K. Percus, and M. Rao. Structure of a liquid-vapor interface. Journal Of Statistical Physics, 17(3):111-136, 1977.

[44] V. M. Kendon. Scaling theory of three-dimensional spinodal turbulence. Physical Review E, 61(6):R6071R6074, June 2000.

[45] V. M. Kendon, M. E. Cates, I. Pagonabarraga, J. C. Desplat, and P. Bladon. Inertial effects in threedimensional spinodal decomposition of a symmetric binary fluid mixture: a lattice boltzmann study. Journal Of Fluid Mechanics, 440:147-203, August 2001.

[46] V. M. Kendon, J. C. Desplat, P. Bladon, and M. E. Cates. 3d spinodal decomposition in the inertial regime. Physical Review Letters, 83(3):576-579, July 1999.

[47] P. Kindt and W. J. Briels. The role of entanglements on the stability of microphase separated diblock copolymers in shear flow. Journal of Chemical Physics, 128(12):124901-1-8, 2007.

[48] J. G. Kirkwood and F. P. Buff. The statistical mechanical theory of surface tension. Journal Of Chemical Physics, 17(3):338-343, 1949.

[49] C. M. Knobler and N. C. Wong. Light-scattering-studies of phase-separation in isobutyric acid + water mixtures .2. test of scaling. Journal Of Physical Chemistry, 85(14):1972-1976, 1981.

[50] S. Koizumi and J. Suzuki. Three-dimensional small-angle neutron scattering of shear-induced phase separation in a dynamically asymmetric polymer mixture. Journal Of Applied Crystallography, 39:878-888, December 2006.

[51] K. A. Koppi, M. Tirrell, F. S. Bates, K. Almdal, and R. H. Colby. Lamellae orientation in dynamically sheared diblock copolymer melts. Journal de Physique II, 2(11):1941, 1992. 
[52] T. Kume and T. Hashimoto. String phase in semidilute polystyrene solutions under steady shear-flow. FlowInduced Structure In Polymers, 597:35-47, 1995.

[53] M. Laradji, O. G. Mouritsen, and S. Toxvaerd. Spinodal decomposition in multicomponent fluid mixtures: A molecular dynamics study. Physical Review E, 53(4):3673-3681, April 1996.

[54] J. Lauger, C. Laubner, and W. Gronski. Correlation between shear viscosity and anisotropic domain growth during spinodal decomposition under shear-flow. Physical Review Letters, 75(19):3576-3579, November 1995.

[55] L. T. Lee, D. Langevin, and B. Farnoux. Neutron reflectivity of an oil-water interface. Physical Review Letters, 67(19):2678-2681, November 1991.

[56] M. P. Lettinga, K. O. Kang, P. Holmqvist, A. Imhof, D. Derks, and J. K. G. Dhont. Nematic-isotropic spinodal decomposition kinetics of rodlike viruses. Physical Review E, 73(1):011412, January 2006.

[57] I. M. Lifshitz and V. V. Slyozov. The kinetics of precipitation from supersaturated solid solutions. Journal Of Physics And Chemistry Of Solids, 19(1-2):35-50, 1961.

[58] P. J. Love, J. B. Maillet, and P. V. Coveney. Three-dimensional hydrodynamic lattice-gas simulations of binary immiscible and ternary amphiphilic flow through porous media. Physical Review E, 6406(6):061302, December 2001.

[59] W. J. Ma, A. Maritan, J. R. Banavar, and J. Koplik. Dynamics of phase-separation of binary fluids. Physical Review A, 45(8):R5347-R5350, April 1992.

[60] B. R. McClain, D. D. Lee, B. L. Carvalho, S. G. J. Mochrie, S. H. Chen, and J. D. Litster. X-ray reflectivity study of an oil-water interface in equilibrium with a middle-phase microemulsion. Physical Review Letters, 72(2):246-249, January 1994.

[61] J. Meunier. In D. Langevin, editor, Light Scattering by Liquid Surfaces and Complementary Techniques, pages 333-574. Marcel Dekker publishers, New York, 1992.

[62] C. A. Miller and P Neogi. Interfacial Phenomena: Equilibrium and Dynamic Effects. Marcel Dekker publishers, New York, 1985.

[63] A. N. Morozov, A. V. Zvelindovsky, and J. G. E. M. Fraaije. Influence of confinement on the orientational phase transitions in the lamellar phase of a block-copolymer melt under shear flow. Physical Review E, 6405(5):051803, November 2001.

[64] N.-T. Nguyen and S. T. Wereley. Fundamentals and Applications of Microfluidics. Artech House Publishers, Norwood, MA, 2002

[65] J. P. Nicolas and N. R. de Souza. Molecular dynamics study of the n-hexane-water interface: Towards a better understanding of the liquid-liquid interfacial broadening. Journal OfChemical Physics, 120(5):24642469, February 2004.

[66] L Onsager. Reciprocal relations in irreversible processes i. Physical Review, 37:405, 1931.

[67] A. Onuki. Phase transitions of fluids in shear flow. Journal Of Physics-Condensed Matter, 9(29):6119-6157, July 1997. 
[68] P. Padilla and S. Toxvaerd. Spinodal decomposition under shear flow. Journal Of Chemical Physics, 106(6):2342-2347, February 1997.

[69] S. Puri and B. Dünweg. Temporally linear domain growth in the segregation of binary fluids. Physical Review A, 45(10):R6977-R6980, May 1992.

[70] S. J. Puri. Surface-directed spinodal decomposition. Journal Of Physics-Condensed Matter, 17(3):R101R142, January 2005.

[71] J. L. Rivera, C. McCabe, and P. T. Cummings. Molecular simulations of liquid-liquid interfacial properties: Water-n-alkane and water-methanol-n-alkane systems. Physical Review E, 67(1):011603, January 2003.

[72] J. Rowlinson and B. Widom. Molecular Theory of Capillarity. Clarendon Press, Oxford, U. K., 1982.

[73] C. P. Royall, D. G. A. L. Aarts, and H. Tanaka. Bridging length scales in colloidal liquids and interfaces from near-critical divergence to single particles. Nature Physics, 3(9):636-640, September 2007.

[74] S. A. Safran. Statistical Thermodynamics of Surfaces, Interfaces and Membranes. Addison-Wesley, Reading, MA, 1994.

[75] M. K. Sanyal, S. K. Sinha, K. G. Huang, and B. M. Ocko. X-ray-scattering study of capillary-wave fluctuations at a liquid surface. Physical Review Letters, 66(5):628-631, February 1991.

[76] M. L. Schlossman. Liquid-liquid interfaces: studied by x-ray and neutron scattering. Current Opinion In Colloid \& Interface Science, 7(3-4):235-243, August 2002.

[77] P. Schofield and J. R. Henderson. Statistical mechanics of inhomogeneous fluids. Proc. R. Soc. London A, 379:231-246, 1982.

[78] S. W. Sides, G. S. Grest, and M. D. Lacasse. Capillary waves at liquid-vapor interfaces: A molecular dynamics simulation. Physical Review E, 60(6):6708-6713, December 1999.

[79] E. D. Siggia. Late stages of spinodal decomposition in binary-mixtures. Physical Review A, 20(2):595-605, 1979.

[80] T. Soddemann, B. Dunweg, and K. Kremer. Dissipative particle dynamics: A useful thermostat for equilibrium and nonequilibrium molecular dynamics simulations. Physical Review E, 68(4):046702, October 2003.

[81] T. M. Squires and S. R. Quake. Microfluidics: Fluid physics at the nanoliter scale. Reviews Of Modern Physics, 77(3):977-1026, July 2005.

[82] P. Stansell, K. Stratford, J. C. Desplat, R. Adhikari, and M. E. Cates. Nonequilibrium steady states in sheared binary fluids. Physical Review Letters, 96(8):085701, March 2006.

[83] J. Stecki and S. Toxvaerd. Equilibrium correlations in interfaces between two immiscible liquids. Journal Of Chemical Physics, 117(6):2860-2866, August 2002.

[84] K. Stratford, J. C. Desplat, P. Stansell, and M. E. Cates. Binary fluids under steady shear in three dimensions. Physical Review E, 76(3):030501, September 2007.

[85] P. Tabeling and D. J. Tildesley. Introduction to Microfluidics. Oxford University Press, Oxford, U. K., 2005. 
[86] H. Tanaka. Unusual phase-separation in a polymer-solution caused by asymmetric molecular-dynamics. Physical Review Letters, 71(19):3158-3161, November 1993.

[87] H. Tanaka. Interplay between wetting and phase separation in binary fluid mixtures: roles of hydrodynamics. Journal Of Physics-Condensed Matter, 13:4637-4674, 2000.

[88] H. Tanaka. Viscoelastic phase separation. Journal Of Physics-Condensed Matter, 12(15):R207-R264, April 2000 .

[89] A. K. Thakre, W. K. den Otter, and W. J. Briels. Domain formation and growth in spinodal decomposition of a binary fluid by molecular dynamics simulations. Physical Review E, 77(1):011503, January 2008.

[90] A. K. Thakre, J. T. Padding, W. K. den Otter, and W. J. Briels. Molecular dynamics simulations of phase separating binary liquids in clylindrical Couette flow. Journal of Chemical Physics, 128:154707, 2008.

[91] S. Toxvaerd. Molecular dynamics simulations of phase separation in chemically reactive binary mixtures. Physical Review E, 53(4):3710-3716, April 1996.

[92] S. Toxvaerd. Molecular dynamics simulations of spinodal decomposition in films of binary mixtures. Physical Review Letters, 83(25):5318-5321, December 1999.

[93] S. Toxvaerd. Dynamics of complex molecular systems: Molecular dynamics simulations of enzyme catalysis, polymer growth and phase separations. Journal Of Molecular Liquids, 84(1):99-110, January 2000.

[94] S. Toxvaerd and J. Stecki. Molecular dynamics of interfaces in opposing fields. Journal Of Chemical Physics, 115(4):1928-1934, July 2001.

[95] S. Y. Trofimov. Thermodynamic consistency in dissipative particle dynamics. PhD thesis, Eindhoven University of Technology, Eindhoven, The Netherlands, 2003.

[96] E. Velasco and S. Toxvaerd. Computer-simulation of late-stage growth in phase-separating binary-mixtures - critical and off-critical quenches. Journal Of Physics-Condensed Matter, 6:A205-A209, June 1994.

[97] N. A. M. Verhaegh, J. S. vanDuijneveldt, J. K. G. Dhont, and H. N. W. Lekkerkerker. Fluid-fluid phase separation in colloid-polymer mixtures studied with small angle light scattering and light microscopy. Physica A, 230(3-4):409-436, September 1996.

[98] A. J. Wagner and J. M. Yeomans. Phase separation under shear in two-dimensional binary fluids. Physical Review E, 59(4):4366-4373, April 1999.

[99] N. C. Wong and C. M. Knobler. Light-scattering studies of phase separation in isobutyric acid + water mixtures. Journal Of Chemical Physics, 69(2):725-735, 1978.

[100] N. C. Wong and C. M. Knobler. Light-scattering-studies of phase-separation in isobutyric acid and water mixtures - hydrodynamic effects. Physical Review A, 24(6):3205-3211, 1981.

[101] R. Yamamoto and X. C. Zeng. Molecular dynamics study of a phase-separating fluid mixture under shear flow. Physical Review E, 59(3):3223-3230, March 1999.

[102] Y. H. Zhang, S. E. Feller, B. R. Brooks, and R. W. Pastor. Computer-simulation of liquid/liquid interfaces .1. theory and application to octane/water. Journal Of Chemical Physics, 103(23):10252-10266, December 1995. 


\section{Acknowledgments}

It is my great pleasure to express my gratitude towards all the people who have been instrumental in the journey of my $\mathrm{PhD}$.

First of all, I would like to thank my promotor, Prof. Wim Briels, for giving me this opportunity to pursue a $\mathrm{PhD}$ in the group and imparting constant enthusiasm and impetus for research, during all these years. I truly appreciate, the help provided by Wouter, his extremely disciplined and organized way of modus operandi, helped me in bringing the thesis in its current form. I also owe a great degree of appreciation for Johan, who has been instrumental in discussions, with his comprehensive, clear and insightful approach to problem solving in research.

I appreciate the support and encouragement I got from my colleagues. Peter and Albert thank you for making coffee table discussions very interesting and also for providing "Tuesday evenings" as a crash course for integration in the Dutch society. Many thanks to Sergey, Yu-Guo, Tanya, Wladimir, Elske, Adrian, Peter M., Carsten, Roberto and specially Marieke for creating a wonderful working atmosphere.

I never felt away from home, thanks to my Indian friends at the University of Twente. Many thanks from heart to Makarand, Kirankumar, Sheela, Ramkrishna, Vijaya, Shrinivas, Ajay, Balaji, Vishnu, Shankar, Sandeep, Jitendra, Srivatsa, Ashok, Chandrashekhar and Mayur; also to affectionate families Pramod and Vishakha, Ravi and Madhavi, Supriyo and Anindita, Manish and Neha, Ram and Veda. I have so many memories to cherish with all of you. A special thanks to Hemant and Nicola for providing an excellent time in latter part of my PhD.

I am indebted to my parents for their constant support and encouragement in difficult moments. Thank you all very much.

Amol Kumar Thakre

September 2008, Enschede 



\section{List of Publications}

- Amol K. Thakre, W.K. den Otter and W.J. Briels

Domain formation and growth in spinodal decomposition of a binary fluid by molecular dynamics simulations

Physical Review E, 77, 011503 (2008)

- Amol K. Thakre, J.T. Padding, W.K. den Otter and W.J. Briels

Molecular dynamics simulations of phase separating binary liquids in cylindrical Couette flow

The Journal of Chemical Physics, 128, 154707 (2008)

- Amol K. Thakre, J.T. Padding, W.K. den Otter and W.J. Briels

Finite system size effect in the interfacial dynamics of binary liquid films

The Journal of Chemical Physics, 129, 044701 (2008)

- Amol K. Thakre, W.K. den Otter, J.T. Padding and W.J. Briels

Spinodal decomposition of asymmetric binary fluids in a micro-Couette geometry simulated with molecular dynamics

The Journal of Chemical Physics, 129, 074505 (2008) 


\section{About the author}

Amol Kumar Thakre was born on May 25th 1978 in Amla, Madhya Pradesh (India). After higher secondary schooling at Amla he went to Madhav Institute of Technology and Science to pursue Bachelors of Engineering and graduated in 2000 and earned first class with distinction in Chemical Engineering. After his Bachelors he moved to Indian Institute of Science, where during his Masters of Science (by research) with Prof. K. S. Gandhi, the author developed a new model to explain phase inversion in liquid-liquid dispersions. In May 2003, the author joined Computational Biophysics (formerly known as Computational Dispersion Rheology) chaired by Prof. Wim Briels to work on phase separation of complex fluids. During his $\mathrm{PhD}$ he participated in various courses, like Han-sur-Lesse (Belgium) winter schools on Macroscopic Physical chemistry, Micro-chemical Systems : Principles and Applications at Delft (Netherlands), Computational Soft Matter : From Synthetic Polymers to Proteins at Bonn (Germany), Complex Physical, Biological and Social Systems and Complex Systems Modeling and Networks at Massachusetts Institute of Technology (USA). During the PhD, he worked on spinodal decomposition of sheared and non-sheared binary liquids, which resulted as publications in various international journals. During this time, he also guided bachelor's students in a simulation project for three years. 